\title{
Dissertação de Mestrado \\ Estudo de determinação dos parâmetros que descrevem a dinâmica de uma supernova galática por um detector de neutrinos futuro.
}

Aluna: Regina Célia Medeiros Felix

Orientadora: Renata Zukanovich Funchal 


\section{Agradecimentos}

À Renata, pela orientação atenciosa e acompanhamento de todo o trabalho e também pela paciência e compreensão nos meus momentos mais difíceis.

Aos meus pais, Maria e Célio, e meus irmãos, Ricardo e Tiago, pelo amor e carinho. Eles que, apesar da distância, estiveram sempre ao meu lado acreditando nas minhas escolhas e torcendo pelo melhor.

À todos os grandes amigos que fiz no IF pelas grandes discussões, idéias e principalmente pelas risadas em todos os nossos encontros. E muito obrigada a Simone e Luciana pela amizade e auxílio constantes.

À Fundação de Amparo a Pesquisa do Estado de São Paulo (FAPESP) pelo apoio financeiro e respeito que guarda aos seus bolsistas. 


\section{Resumo}

O objetivo desta dissertação foi estudar os sinais que neutrinos provenientes de supernovas galáticas poderão produzir em detectores futuros, através de simulações de eventos observados na Terra por um detector Cherenkov pela reação de decaimento beta inverso.

Por ser um local único em que neutrinos se encontram em condições de equilíbrio térmico, a física de neutrinos de supernova pode ser fonte de novo conhecimento na física de partículas elementares. Iniciamos o trabalho apresentando os aspectos mais importantes da física de neutrinos tal como é conhecida hoje, seguido de um estudo do papel do neutrino na explosão de uma supernova do tipo II e a influência das oscilações em futuras observações.

As simulações foram feitas primeiramente considerando uma supernova de potencial estático, com a determinação de limites nos principais parâmetros que descrevem sua dinâmica. Utilizamos os casos de hierarquia normal e inversa e ângulos de mistura nos limites totalmente adiabático e não-adiabático. Posteriormente consideramos uma supernova de potencial dinâmico, a partir da qual estudamos o comportamento das probabilidades de transição e o perfil do espectro detectado nos mesmos casos anteriores. Com este potencial também foi possível observar o comportamento temporal do espectro e como este pode ser modificado com a hierarquia e ângulo de mistura.

Mostramos que em uma futura detecção, o número de eventos e consequentemente sua variação com parâmetros de supernova não terão interferência considerável do efeito de onda de choque. Contudo, este pode causar distorções no espectro energético e temporal que poderão ter papel importante na determinação da hierarquia de massa e maior delimitação do ângulo de mistura. 


\section{Abstract}

The goal of this dissertation is to study the signals that supernova neutrinos could produce in future detectors, through simulations of events observed on Earth by a Cherenkov detector and inverse beta decay reaction.

Since a supernova has been the only situation in which neutrinos are able to reach thermal equilibrium, the physics of supernova neutrinos can be source of a new knowledge in physics of elementary particles. We begin this work presenting the most important aspects of neutrino physics as known today, and then studying the role of neutrino in a type II supernova explosion and the oscillation influence in future observations.

The simulations were initially performed considering a static potential, defining limits for the main parameters that describe its dynamics. We considered the cases of normal and inverse mass hierarchy and mixing angles within fully adiabatic and non-adiabatic limits. Later we used a supernova dynamic potential to study behaviour of transitions probabilities and the profile of the spectrum detected in these previous cases. From this potential we also observed the temporal behaviour of the spectrum and how it can be modified with the hierarchy and the mixing angle.

We show that, in a future detection, the number of events and hence their variations with supernova parameters, will not suffer interference of the shock wave effect. However, this effect can cause distortions in the energy and time spectrum that could have an important role in determining the mass hierarchy and better constraints for the mixing angle. 


\section{Sumário}

$\begin{array}{ll}\text { Introdução } & 1\end{array}$

1 Massa, mistura e oscilação de Neutrinos $\quad 3$

1.1 Breve apresentação aos neutrinos . . . . . . . . . . . . . . . . . . . . 3

1.2 Neutrinos massivos e o Modelo Padrão . . . . . . . . . . . . . . . . . . . . . . 4

1.2.1 Neutrinos de Dirac e Majorana . . . . . . . . . . . . . . . . . . . 5

1.2 .2 Duplo decaimento beta . . . . . . . . . . . . . . 8

1.3 Evidências de oscilação . . . . . . . . . . . . . . . . . . . . . . 11

1.3 .1 Neutrinos solares . . . . . . . . . . . . . . . . . . . . . . 11

1.3.2 Neutrinos atmosféricos . . . . . . . . . . . . . . . . . . . 13

1.3.3 Neutrinos de reatores e aceleradores . . . . . . . . . . . . . . . . . . 14

1.4 Oscilação no vácuo . . . . . . . . . . . . . . . . . . . . . . . . . . . . 14

1.5 Oscilação na matéria . . . . . . . . . . . . . . . . . . . . . . 17

1.5 .1 Efeito MSW . . . . . . . . . . . . . . . . . . . 19

1.5.2 Condição de adiabaticidade . . . . . . . . . . . . . . . . . . . . . . 19

1.6 Oscilação dominante em neutrinos solares . . . . . . . . . . . . . . . . . . . . 20

1.7 Oscilação dominante em neutrinos atmosféricos . . . . . . . . . . . . . . . 23

2 Neutrinos de Supernova $\quad 26$

2.1 Mecanismo de explosão de supernovas . . . . . . . . . . . . . . . . . 26

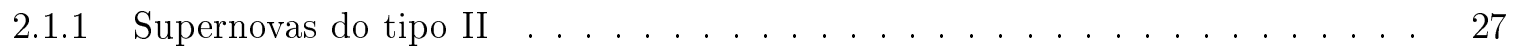

2.2 Evolução do fluxo de neutrinos . . . . . . . . . . . . . . . . . . . . 28

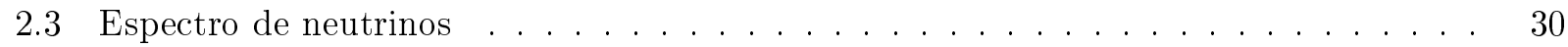

2.3.1 Neutrinos e antineutrinos do elétron . . . . . . . . . . . . . . . . . 30 
2.3.2 Neutrinos não eletrônicos . . . . . . . . . . . . . . . . . . . . 30

2.3.3 Distorção espectral (pinching) . . . . . . . . . . . . . . . . . . . . . 31

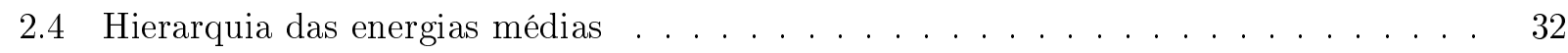

2.5 Dinâmica de conversão . . . . . . . . . . . . . . . . . . . . . . 34

2.5.1 Regiões de conversão . . . . . . . . . . . . . . . . . . 34

2.5.2 Espectro de massa . . . . . . . . . . . . . . . . . . . . . 34

2.5 .3 Fatorização da dinâmica . . . . . . . . . . . . . . . . . . . . 36

2.5.4 Limites de adiabaticidade . . . . . . . . . . . . . . . . . . 36

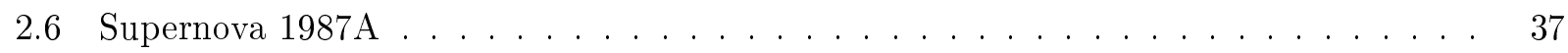

3 Oscilação de neutrinos em supernovas $\quad 40$

3.1 Perfil de densidades . . . . . . . . . . . . . . . . . . . . . . . . . . 40

3.2 Transição entre duas famílias . . . . . . . . . . . . . . . . . . . . . 41

3.3 Transição entre três famílias . . . . . . . . . . . . . . . . . . . . . . . . 44

3.3.1 Transições com hierarquia normal para neutrinos . . . . . . . . . . . . 44

3.3.2 Transições com hierarquia normal para antineutrinos . . . . . . . . . . . 46

3.3.3 Transições com hierarquia inversa para neutrinos . . . . . . . . . . . 46

3.3.4 Transições com hierarquia inversa para antineutrinos . . . . . . . . . . . . 47

3.4 Transformação de sabor coletiva em neutrinos de supernova . . . . . . . . . . . . . 47

4 Estudo de oscilação de neutrinos para supernovas galáticas com um potencial $\begin{array}{ll}\text { estático } & 49\end{array}$

4.1 Definições iniciais . . . . . . . . . . . . . . . . . . . . . . . . . . . . . 49

4.2 Número de eventos total e espectro energético esperado . . . . . . . . . . . . . . . . 52

4.3 Dependência do número de eventos com os parâmetros da supernova . . . . . . . . . 54

4.4 Casos estudados . . . . . . . . . . . . . . . . . . . . . . . . 55

4.5 Estudo preliminar da dependência do número total de eventos com os parâmetros de

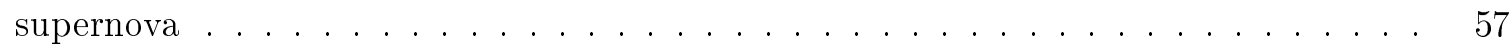

4.6 Estudo da avaliação da precisão esperada para determinação dos parâmetros de su-

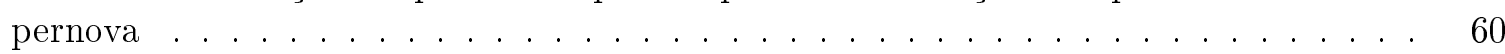


5 Estudo do efeito de uma onda de choque em supernovas galáticas no espectro dos neutrinos observados na Terra

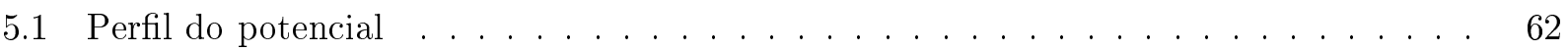

5.2 Parametrização empírica do perfil de densidades . . . . . . . . . . . . . . . . 63

5.3 Probabilidades de transição . . . . . . . . . . . . . . . . . . . . 65

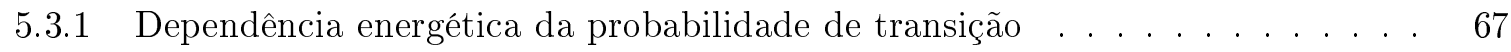

5.3.2 Dependência temporal da probabilidade de transição . . . . . . . . . . . . . . 71

5.4 Espectro observado . . . . . . . . . . . . . . . . . . . . . 75

$\begin{array}{ll}\text { Conclusões } & 81\end{array}$ 


\section{Lista de Figuras}

1.1 Duplo decaimento beta (a) com e (b) sem neutrinos, respectivamente. . . . . . . . 9

1.2 Fluxo de neutrinos predito pelo MPS em função da energia. . . . . . . . . . . . . . . 12

1.3 Valores característicos de L e E para diferentes fontes de neutrinos e os correspondentes

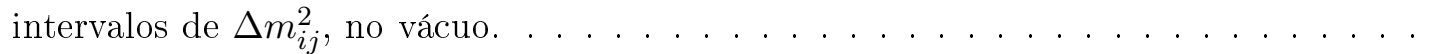

1.4 Região dos parâmetros de oscilação (com 90\%, 95\%, 99\% e 99,73\% de confiança) compatíveis com a análise das taxas totais dos experimentos com $\mathrm{Cl}$, Ga e dos experimentos de espalhamento SK e SNO $[63] . \ldots \ldots \ldots \ldots \ldots$

1.5 Região dos parâmetros de oscilação (com 90\%, 95\% e 99\% de confiança) obtida pela análise global dos dados de neutrinos solares $[63] \ldots \ldots \ldots \ldots$. . . . . . . . . .

1.6 Região dos parâmetros de oscilação (com 90\%, 95\% e 99\% de confiança) obtida pela análise dos dados de KamLAND [63]. . . . . . . . . . . . . . . . .

1.7 Região dos parâmetros de oscilação (com 90\%, 95\% e 99\% de confiança) obtidas pela análise combinada dos dados solares e KamLAND [63] . . . . . . . . . . . . . . .

1.8 Região de parâmetros de oscilação (com 90\%, 95\%, 99\% e 99,73\% de confiança) obtida pela análise dos dados de neutrinos atmosféricos. . . . . . . . . . . . . .

1.9 Região dos parâmetros de oscilação obtida pela análise dos dados de K2K (esquerda) e MINOS (direita), com 90\%, 95\% e 99\% de confiança. As linhas contínuas não preenchidas sobrepostas correspondem as mesmas regiões para dados atmosféricos [63]. 25

2.1 Luminosidade de neutrinos instantes após a explosão de uma supernova do tipo II. " $\nu_{\mu}$ " refere-se a (anti)neutrinos que não sejam do elétron [77]. . . . . . . . . . . .

2.2 Três fases principais da evolução do fluxo de neutrinos: erupção do choque (crescimento rápido), acreção e resfriamento do manto [78] . . . . . . . . . . . . . . . .

2.3 Distribuição do número $\mathrm{N}$ de eventos de $\mathrm{CC}$ para (a) um espectro térmico ( $\mathrm{T}=3 \mathrm{MeV}$, $\eta=0)$, e (b) um espectro distorcido (pinched) $(\mathrm{T}=3 \mathrm{MeV}, \eta=3)[82] . \ldots . . .$.

2.4 Resultados de diferentes simulações para evolução da energia média dos neutrinos [84, 85, 87]. Fica clara a diferença de resultados entre as simulações dos dois primeiros quadros (a) e (b), que utilizam descrição simplificada de $\nu_{x}$ para a do terceiro quadro (c), com descrição mais completa. . . . . . . . . . . . . . . . . . . 
2.5 Diagrama de ressonâncias para hierarquias normal e inversa. . . . . . . . . . . .

2.6 Contornos de igual probabilidade de transição $\left(P_{J}\right)$ através de regiões de ressonância com diferentes parâmetros de mistura $\left(\Delta m^{2}, \theta\right)$. Contornos da direita vão de $P_{J}=$ 0,02 a 0,1 e da esquerda de $P_{J}=0,9$ a 0,98 . Estas probabilidades de transição foram obtidas para um perfil de densidades simples: $\rho(r) Y_{e}=2 \times 10^{4} \mathrm{~g} / \mathrm{cm}^{3}\left(r / 10^{9} \mathrm{~cm}\right)^{3}$ [77]. 36

2.7 Disposição dos detectores Kamiokande II e IMB na Terra no momento do sinal da SN 1987A.

2.8 Eventos detectados por K2, IMB e Baksan. Nesta montagem, o primeiro neutrino detectado em cada experimento foi condiderado com $t=0 \ldots \ldots \ldots$. . . . .

3.1 Curvas de nível mostram a dependência da probabilidade de transição do neutrino, $P_{c}$, e da probabilidade de sobrevivência, $P_{e e}$, com os parâmetros de mistura $\left(\Delta m^{2}, \tan ^{2} \theta\right)$ para transições entre dois sabores e energia do neutrino $E=15 \mathrm{MeV}$. . . . . . . .

3.2 Espectro de neutrinos na neutrinosfera (linhas finas) e após atravessarem a região densa (linhas grossas). As linhas sólidas representam neutrinos e as pontilhadas os antineutrinos. Espectro positivo corresponde a (anti)neutrinos eletrônicos e espectro negativo corresponde a (anti)neutrinos $x[128] \ldots \ldots \ldots \ldots$

4.1 Probabilidade de sobrevivência de neutrinos $\left(P_{e e}\right)$ e antineutrinos $\left(P_{\overline{e e}}\right)$ do elétron para hierarquia normal. O cálculo foi feito para energia $E=60 \mathrm{MeV}$. Consideramos aqui $\operatorname{sen}^{2} \theta_{12}=0,31, \Delta m_{21}^{2}=8,0 \times 10^{-5} \mathrm{eV}^{2}$ e $\left|\Delta m_{31}^{2}\right|=3,0 \times 10^{-3} \mathrm{eV}^{2}[140] . \ldots$

4.2 Análogo à Fig. (4.1) mas para hierarquia inversa. . . . . . . . . . . . . . . . . .

4.3 Seção de choque para o decaimento beta inverso até primeira ordem de teoria de perturbação [144].

4.4 Espectro de energia de antineutrinos que se espera observar em um detector Cherenkov de 540 kton e eficiência de $100 \%$ para observação do neutrino através da reação de decaimento beta inverso no intervalo de tempo de $6 \pm 0,25 \mathrm{~s}$. O gráfico a esquerda corresponde ao ângulo de mistura $\operatorname{sen}^{2} \theta_{13}=10^{-3}$ e o gráfico a direita a $\operatorname{sen}^{2} \theta_{13}=$ $10^{-6}$. Ambos mostram os casos de hierarquia normal e inversa. . . . . . . . . . . . .

4.5 Variação do número de eventos $N_{T O T}$ como função das energias médias do antineutrino do elétron dos neutrinos não-eletrônicos e de $E_{b} / D^{2}$, respectivamente. Estamos considerando hierarquia normal e ângulo de mistura grande e os parâmetros da SN fixos segundo a Tabela $(4.1) \ldots \ldots \ldots \ldots \ldots \ldots$

4.6 Distribuição do número de eventos de $\bar{\nu}_{e}$ para intervalos de energia de $10 \mathrm{MeV}$. O primeiro caso corresponde a ângulo de mistura grande, $\operatorname{sen}^{2} \theta_{13}=10^{-3}$, e HN (caso equivalente a ângulo de mistura pequeno, $\operatorname{sen}^{2} \theta_{13}=10^{-6}$. O segundo caso se refere tambem a ângulo de mistura grande com HI, A barra de erro apresentada refere-se apenas à incerteza estatística multiplicada por 10. Os parâmetros fixos são os mesmos

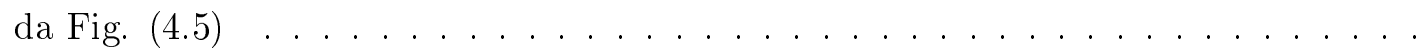

4.7 Sensibilidade do detector para os parâmetros $\left\langle E_{\bar{e}}\right\rangle,\left\langle E_{x}\right\rangle$ e $E_{b} / D^{2}$ de supernova dois a dois em hierarquia normal e ângulo de mistura grande, $\operatorname{sen}^{2} \theta_{13}=10^{-3} \ldots$. . . . 
4.8 Sensibilidade do detector para os parâmetros $\left\langle E_{x}\right\rangle$ e $E_{b} / D^{2}$ de supernova em hierarquia inversa e ângulo de mistura grande, $\operatorname{sen}^{2} \theta_{13}=10^{-3} \ldots \ldots$. . . . . . . . . . .

4.9 Sensibilidade do detector para os parâmetros $\left\langle E_{\bar{e}}\right\rangle,\left\langle E_{x}\right\rangle$ e $E_{b} / D^{2}$ de supernova em hierarquia normal e ângulo de mistura grande, $\operatorname{sen}^{2} \theta_{13}=10^{-3} \ldots$. . . . . . . . . .

4.10 Sensibilidade do detector para os parâmetros $\left\langle E_{x}\right\rangle$ e $E_{b} / D^{2}$ de supernova em hierarquia inversa e ângulo de mistura grande, $\operatorname{sen}^{2} \theta_{13}=10^{-3}$

5.1 Perfil de densidade simulado para uma supernova com onda de choque como função do raio para diferentes tempos [149]

5.2 Potencial de matéria em função da distância radial do centro da supernova para tempos diferentes $(t=1-8 \mathrm{~s}$ de cima para baixo). É fácil perceber que podem existir até três pontos com o mesmo potencial $\mathrm{V}$, pois há uma queda no potencial no ponto em que se encontra a frente de onda. . . . . . . . . . . . . . . . . . .

5.3 Probabilidades de transição $P_{H}(E)$ após t=4 s da explosão e diferentes ângulos de mistura. Em cada quadro estão representadas também as componentes $P_{1,2, s, 3}$. . .

5.4 Probabilidades de transição $P_{H}(E)$ após $\mathrm{t}=4 \mathrm{~s}$ da explosão e diferentes ângulos de mistura. Em cada quadro estão representadas também as componentes $P_{1,2, s, 3}$. . . .

5.5 Probabilidades de transição $P_{H}(E)$ após t=4 s da explosão e ângulo de mistura indicado. Estão representadas também as componentes $P_{1,2, s, 3} \ldots \ldots$. . . . . . . . . 70

$5.6 P_{H}(E)$ após $t=0,4$ e $8 \mathrm{~s}$ para $\operatorname{sen}^{2} \theta_{13}=10^{-4} \ldots \ldots \ldots \ldots \ldots \ldots$

5.7 Probabilidades de transição $P_{H}(t)$ para $\mathrm{E}=30 \mathrm{MeV}$ e diferentes ângulos de mistura. Em cada quadro estão representadas também as componentes $P_{1,2, s, 3}$. . . . . . . . .

5.8 Probabilidades de transição $P_{H}(t)$ para $\mathrm{E}=30 \mathrm{MeV}$ e diferentes ângulos de mistura. Em cada quadro estão representadas também as componentes $P_{1,2, s, 3}$. . . . . . . . .

5.9 Probabilidades de transição $P_{H}(t)$ para $\mathrm{E}=30 \mathrm{MeV}$ e ângulos de mistura indicado. Estão representadas também as componentes $P_{1,2, s, 3} \ldots \ldots \ldots$. . . . . . . .

5.10 Espectro de energia de antineutrinos eletrônicos de uma supernova galática, provenientes de decaimento beta inverso, no intervalo de tempo $t=6 \pm 0,25 \mathrm{~s} \mathrm{e} \mathrm{sen}^{2} \theta_{13}=10^{-6}$, $10^{-5}$ e $10^{-3}$ respectivamente. . . . . . . . . . . . . . . . .

5.11 Espectro de energia de antineutrinos eletrônicos de uma supernova galática a cada 0,5 s de 6 a 9 s após a explosão. Os dados são provenientes de decaimento beta inverso para diferentes valores de ângulo de mistura. Os pontos correspondem a hierarquia

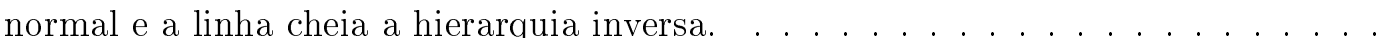

5.12 Dependência temporal do número de eventos de supernova para diferentes intervalos de energia (de cima para baixo: $30 \pm 5,40 \pm 5,50 \pm 5$ e $60 \pm 5 \mathrm{MeV}$ ). Os pontos correspondem a hierarquia normal e as linhas a hierarquia inversa. Os quadros correspondem respectivamente aos ângulos de mistura: $\operatorname{sen}^{2} \theta_{13}=10^{-6}, 10^{-5}, 10^{-4} \mathrm{e}$

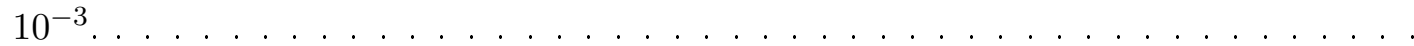




\section{Lista de Tabelas}

4.1 Parâmetros da SN utilizados para as simulações. . . . . . . . . . . . . . . 55

4.2 Número esperado de eventos de supernova em um detector Cherenkov de 540 kton para reação de decaimento beta inverso para o ponto no espaço de parâmetros definido na

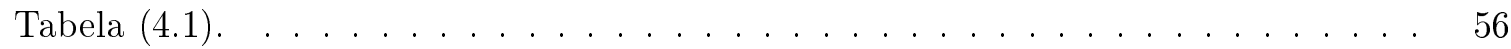

5.1 Número esperado de eventos de supernova em um detector Cherenkov de $540 \mathrm{kton}$ para reação de decaimento beta inverso referente a um ponto no espaço de parâmetros definido na Tabela (4.1). Vemos aqui a comparação de resultados para o caso sem onda de choque (V estático), com onda de choque (V dinâmico), a variação absoluta da quantidade de eventos esperada por cada modelo e o número de eventos correspondendo a uma diferença de $5 \sigma$ a partir do potencial estático. . . . . . . . . 


\section{Introdução}

Os testes experimentais pelos quais os neutrinos vêm sendo submetidos já forneceram grandes avanços para nosso entendimento de sua física, tais como as evidências de massas e as confirmações, por diversos experimentos, do fenômeno de oscilação com a consequente determinação dos parâmetros de mistura com alguma precisão. Este grande desenvolvimento em conjunto com o fato dos neutrinos estarem inclusos no Modelo Padrão [1] apenas como partículas sem massa leva a acreditar que a física de neutrinos se apresenta como uma das principais motivações para o desenvolvimento de uma nova física além do Modelo Padrão.

Dentre as possíveis fontes de neutrinos que podem agregar novos conceitos e resultados experimentais, os neutrinos provenientes de supernovas são eventos de detecção rara, principalmente porque são esperadas apenas três explosões de supernova na galáxia a cada século. Apenas em 1987 foi possível detectar 20 neutrinos que coincidem temporalmente com a SN1987A ocorrida na Grande Nuvem de Magalhães.

A importância do estudo de neutrinos de supernova e sua detecção encontra-se principalmente no fato de que o núcleo de uma estrela em colapso corresponde ao único local no universo atual em que pode-se considerar os neutrinos em equilíbrio térmico, sendo assim, devido as condições em que se encontram, fonte de informação tanto com relação à física de neutrinos quanto ao processo de colapso estelar e, indiretamente, do universo primordial.

A expectativa por uma próxima explosão de supernova na galáxia fez aumentar a quantidade de trabalhos que buscam predizer o sinal que poderá ser detectado e, a partir deste, saber o quanto este sinal pode acrescentar no entendimento atual de neutrinos. Resultados como um melhor limite para o ângulo de mistura $\theta_{13}$ e definição da hierarquia de massa estão entre os mais esperados.

Com o recente desenvolvimento de novas abordagens e a descoberta de novos efeitos na oscilação em meios com altas densidades de neutrinos $[124,125]$, a física de neutrinos de supernovas passa a um papel de destaque na física de partículas elementares.

O objetivo do nosso trabalho foi estudar os sinais que neutrinos provenientes de supernovas galáticas poderão produzir em detectores futuros. Para o cálculo do fluxo de neutrinos investigamos diferentes modelos para o potencial de matéria da estrela durante o colapso. Com esse objetivo, fizemos um trabalho de atualização do que hoje é conhecido da física de neutrinos, oscilações, geração de masa, limites de parâmetros e detectores, seguido de um estudo do papel do neutrino na explosão de uma supernova, observando os pontos em que a física de neutrinos, ainda em desenvolvimento, tem importância crucial e o papel das oscilações nos resultados de futuras observações.

Posteriormente fizemos simulações de eventos observados na Terra por um detector Cherenkov 
atravéz da reação de decaimento beta inverso. Estas simulações foram feitas considerando uma supernova de potencial estático e em seguida para uma supernova de potencial dinâmico, ou seja, com efeitos da passagem de uma onda de choque. Desta forma, pudemos avaliar a magnitude e importância deste efeito em estudos e deteç̧ões futuras.

O trabalho apresentado segue em 5 partes. No capítulo 1 apresentamos a física de neutrinos tal como conhecida hoje e suas principais perspectivas.

No capítulo 2 estudamos o mecanismo da explosão de supernovas do tipo II, detalhando a influência dos neutrinos em cada etapa do colapso gravitacional. Discutimos também os resultados obtidos a partir dos eventos da SN1987A.

No capítulo 3 descrevemos uma abordagem analítica aproximada para o cálculo de oscilação de neutrinos de supernovas baseada em uma probabilidade de transição de dupla exponencial. Os resultados obtidos neste capítulo foram utilizados nos capítulos posteriores.

No capítulo 4 investigamos a dependência energética do espectro de neutrinos para uma supernova de potencial estático, avaliando a possibilidade de obter limites para parâmetros de supernova, fixos os parâmetros de oscilação, a partir de uma futura detecção.

No capítulo 5 o cálculo anterior é refinado para o caso de uma supernova com a passagem de uma onda de choque, tornando-se relevante a dependência temporal do espectro e as mudanças no potencial devido a variação da densidade causada pela onda. Enfim, concluimos com uma comparação entre os resultados e sua relevância para a física de neutrinos. 


\section{Capítulo 1}

\section{Massa, mistura e oscilação de Neutrinos}

Neutrinos são partículas que têm se destacado desde o início pelo seu comportamento e propriedades diferentes dos léptons carregados. Além de interagirem apenas fracamente, podendo assim atravessar grandes regiões de matéria sem qualquer desvio, dependendo de sua fonte de emissão vão possuir energia em um intervalo bem definido, cujo espectro dependerá dos processos de emissão, eventualmente do meio de propagação e da forma de deteç̧ão.

Há diversas evidências experimentais que neutrinos tem massa e mistura e sofrem oscilação de sabor [1]. A melhor determinação dos parâmetros que regem estas oscilações é essencial para um estudo preciso de suas propriedades e a compreensão de seu papel no conjunto das partículas elementares e no Modelo Padrão (MP), que, apesar de todo sucesso experimental, hoje engloba apenas neutrinos não massivos.

Neste capítulo daremos uma visão geral do que hoje é conhecido e em que ponto estão as pesquisas e resultados experimentais para a descrição de oscilação de neutrinos. São estes resultados que justificam assumir oscilação de neutrinos nos capítulos que seguem.

\subsection{Breve apresentação aos neutrinos}

Em 1989, a medida da largura invisível do bóson $Z^{0}$ [1] forneceu a primeira evidência experimental com grande precisão da existência de apenas três espécies de neutrinos ativos com massa inferior a massa do $\mathrm{Z}^{0}\left(M_{\mathrm{Z}^{0} / 2}\right)$. Porém, a hipótese da existência dessas partículas que interagem fracamente teve origem quase 60 anos antes a partir de uma idéia simples.

Wolfgang Pauli propôs, em 1930, a existência de uma partícula de massa nula, ou quase nula e spin $\frac{1}{2}$ para que o princípio da conservação da energia não fosse violado no decaimento nuclear beta que, até então mostrava um espectro contínuo ao contrário do esperado. A deteç̧ão desta partícula, contudo, mostrou-se difícil para os detectores e tecnologia da época, uma vez que, em 1934, Hans Bethe e Rudolf Peierls [2] mostraram que a seção de choque entre neutrino e matéria era tão pequena que ele poderia atravessar a Terra sem desvio.

O projeto de detecção do neutrino a partir de uma fonte nuclear surgiu e, em 1956 [3] Frederick Reines e Clyde Cowan realizaram um experimento que captava neutrinos do reator de Savannah 
River na Carolina do Sul, EUA. Os anti-neutrinos vindos do reator interagem com os prótons de uma mistura de 400 litros de água com cloreto de cádmio, gerando um pósitron e um nêutron. O primeiro é aniquilado por um elétron, emitindo assim dois fótons de $0,5 \mathrm{MeV}$ em direções opostas. Simultaneamente o nêutron é absorvido pelo cádmio ${ }^{108} \mathrm{Cd}$ produzindo um estado excitado ${ }^{109} \mathrm{Cd}$ que emite um fóton,

$$
n+{ }^{108} \mathrm{Cd} \rightarrow{ }^{109} \mathrm{Cd}^{*} \rightarrow{ }^{109} \mathrm{Cd}+\gamma
$$

A deteç̧ão do fóton adicional cerca de $5 \times 10^{-6}$ segundos após a aniquilação elétron-pósitron gera uma assinatura para a reação do neutrino.

Em 1968, Gribov e Pontecorvo [4,5] mostraram que a existência de uma massa para o neutrino implicava na possibilidade de oscilação entre os sabores. O desaparecimento de neutrinos atmosféricos $\left(\nu_{\mu}\right.$ 's) e neutrinos solares $\left(\nu_{e}\right.$ 's) poderia ser facilmente explicado em termos dessa oscilação. Contudo, neste novo cenário, para a oscilação de dois neutrinos, pelo menos um deveria ser massivo.

No Modelo Padrão neutrinos foram introduzidos como férmions sem massa, e nenhum termo de massa renormalizável invariante de gauge pode ser construido sem a introdução da componente de mão-direita. Desta forma, a evidência de massa, oscilação ou violação CP no setor de neutrinos são indicação forte de nova física.

Como já mencionado, hoje sabemos que existem três tipos de neutrinos ativos. A existência do neutrino do múon $\left(\nu_{\mu}\right)$ e do tau $\left(\nu_{\tau}\right)$ era esperada pela propriedade de correspondência com seus pares carregados. O neutrino do múon foi descoberto em 1962 pelo grupo de Lederman, Schwartz e Steinberger [6] e, após a descoberta do terceiro lépton carregado (tau), em meados da década de 70, iniciou-se também a tentativa de deteç̧ão de seu neutrino correspondente, realizada em 2001 pelo experimento DONUT do Fermilab [7].

Além de serem produzidos por reatores nucleares e aceleradores, as maiores fontes de neutrinos são naturais. Neutrinos podem vir de interações de raios cósmicos na atmosfera (neutrinos atmosféricos), podem ser produzidos em reações termonucleares no interior de uma estrela (neutrinos estelares), os que chegam à Terra provenientes da estrela mais próxima são os chamados neutrinos solares, e neutrinos relíquias do Big Bang. Devido a sua abundância, neutrinos têm um papel importante no estudo da astrofísica e cosmologia. Por exemplo, eles carregam $99 \%$ da energia emitida em supernovas do tipo II, além de ter um papel crucial no mecanismo de explosão dessas estrelas [8].

\subsection{Neutrinos massivos e o Modelo Padrão}

O Modelo Padrão foi construído de forma que os neutrinos não sejam partículas massivas. Sua simetria, $S U(2)_{L} \times U(1)_{Y}$, fixa apenas a maneira como os férmions e os bósons de gauge do modelo se transformam. A massa dos férmions carregados aparece através do mecânismo de quebra de simetria (Mecânismo de Higgs), porém, para neutrinos, a massa continua nula em qualquer ordem perturbativa.

Neutrinos são férmions que não possuem interações forte nem eletromagnética. Neutrinos ativos possuem interação fraca e aparecem em dubletos de $S U(2)_{L}$. No MP temos três neutrinos ativos, que residem em dubletos leptônicos 


$$
L_{l}=\left(\begin{array}{c}
\nu_{l L} \\
l_{L}^{-}
\end{array}\right), \quad l=e, \mu, \tau
$$

sendo $e, \mu, \tau$ os autoestados de massa dos léptons carregados. No MP a componente de mão-direita dos férmions carregados é introduzida como singleto do grupo de simetria $S U(2)$ e neutrinos de mão-direita não são introduzidos. No modelo mínimo existem três famílias de férmions e um dubleto de Higgs complexo $\phi \equiv\left(\begin{array}{c}\phi^{+} \\ \phi^{0}\end{array}\right)$.

Após a quebra espontânea de simetria [9], a lagrangeana para campos fermiônicos gera dois termos de interação para neutrinos. O termo de correntes carregadas $(\mathrm{CC})$

$$
-\mathcal{L}_{\mathrm{CC}}=\frac{g}{\sqrt{2}} \sum_{l} \overline{\nu_{l L}} \gamma^{\mu} l_{L} W_{\mu}+\text { h.c. }
$$

e o termo de correntes neutras $(\mathrm{CN})$

$$
-\mathcal{L}_{\mathrm{CN}}=\frac{g}{2 \cos \theta_{W}} \sum_{l} \overline{\nu_{l L}} \gamma^{\mu} \nu_{l L} Z_{\mu}^{0}+\text { h.c. }
$$

sendo $\gamma^{\mu}$ as matrizes de Dirac, $\theta_{W}$ o ângulo de Weinberg e $g$ a constante de acoplamento fraco.

Estas equações fornecem todas as interações dos neutrinos no MP. A partir da Eq. (1.4) é determinada a largura do decaimento do bóson $Z^{0}$ em neutrinos, que é proporcional ao número de neutrinos ativos de mão esquerda. O resultado experimental mais recente para este número é $N_{\nu}=2,984 \pm 0,008$ [1], evidenciando assim a existência de apenas 3 neutrinos ativos leves na natureza.

\subsubsection{Neutrinos de Dirac e Majorana}

Todos os demais férmions - léptons carregados e quarks - são partículas de Dirac, ou seja, obedecem as equações de Dirac e são descritos por um spinor complexo de quatro componentes. Se neutrinos não tivessem massa seriam bem descritos por spinores complexos de duas componentes, os spinores de Weyl. Porém, uma vez que temos indicações de que neutrinos são massivos, eles não podem ser assim descritos e, como qualquer outro férmion deveria possuir massa de Dirac. Contudo, uma diferença é crucial entre os demais férmions e os neutrinos: estes não possuem carga elétrica, nos abrindo assim a possibilidade para que possam ser descritos por spinores de Majorana.

Assumindo neutrinos como partículas massivas é necessário que o MP seja extendido de forma a acomodar esta característica. Quando a análise é feita assumindo neutrinos não massivos, o MP não contém o campo de mão direita do neutrino $\left(\nu_{R}\right)$, e sim apenas o campo de mão esquerda $\left(\nu_{L}\right)$ que acopla com os bósons $\mathrm{W}$ e Z. Para acomodar a massa do neutrino da mesma maneira que os demais férmions é necessário adicionar este campo de mão direita, construindo assim um termo de massa de Dirac.

Este termo conserva o chamado número leptônico L, simetria global que distingue neutrinos e léptons carregados negativamente de antineutrinos e léptons carregados positivamente. Uma vez que 
tudo mais no MP conserva $L$, temos um mundo em que $L$ é uma simetria que se conserva. Sendo os neutrinos partículas sem carga eletromagnética, os autoestados de massa dos neutrinos $\nu_{i}$ se diferem de sua antipartícula apenas pelo número leptônico $L\left(\bar{\nu}_{i}\right)=-L\left(\nu_{i}\right)$.

Diferentemente da simetria U(1) eletromagnética, a simetria U(1) do número leptônico global não governa a dinâmica. Desta forma, não é uma simetria essencial ao modelo e, se violada, não há razão para que um $\nu_{L}$ não possa ser um $\bar{\nu}_{R}$ no caso de um boost. Assim, pode ser necessário apenas um campo fermiônico de duas componentes, como proposto por Majorana [10] em 1937, para descrever o neutrino.

Concluindo, uma partícula e uma antipartícula são definidas a partir de alguns números quânticos que se conservam. Se, como no caso dos neutrinos, não há número quântico que as diferencia, então partícula e antipartícula são idênticas, de forma que um neutrino de Majorana é o seu próprio antineutrino.

\section{Termos de massa}

Suponhamos que sejam adicionados ao MP neutrinos de mão direita necessários ao termo de massa de Dirac. Exigindo a conservação do número leptônico global, termos de massa de Majorana não devem ser adicionados. Contudo, excluindo esta imposição, os termos de Majorana podem estar presentes.

O cálculo a seguir é feito para uma geração de neutrinos, e pode ser facilmente extendido para as demais gerações.

Definimos conjugação de carga (C) de um campo $\nu$ por [9]

$$
\nu^{C}=C \gamma^{0} \nu^{*}=i \gamma^{2} \nu^{*},
$$

e componentes quirais

$$
\nu_{L}=\frac{1}{2}\left(1-\gamma_{5}\right) \nu ; \quad \nu_{R}=\frac{1}{2}\left(1+\gamma_{5}\right) \nu,
$$

com a notação

$$
\nu_{L}^{C} \equiv\left(\nu_{L}\right)^{C}=\frac{1}{2}\left(1+\gamma_{5}\right) \nu^{C}=\left(\nu^{C}\right)_{R} .
$$

A massa dos férmions conecta campos de mão esquerda e direita. Massas de Dirac conectam componentes L e R do mesmo campo (sendo $\nu=\nu_{L}+\nu_{R}$ )

$$
-\mathcal{L}_{D}=D\left(\bar{\nu}_{L} \nu_{R}+\bar{\nu}_{R} \nu_{L}\right)=D \bar{\nu} \nu .
$$

Já um termo de massa de Majorana conecta componentes de mão esquerda e direita de campos conjugados 


$$
\begin{aligned}
-\mathcal{L}_{M A}=A\left(\bar{\nu}_{L}^{C} \nu_{L}+\bar{\nu}_{L} \nu_{L}^{C}\right) & =A \bar{\chi} \chi \\
-\mathcal{L}_{M B}=B\left(\bar{\nu}_{R}^{C} \nu_{R}+\bar{\nu}_{R} \nu_{R}^{C}\right) & =B \bar{w} w
\end{aligned}
$$

sendo agora os auto estados de massa os campos: $\chi=\nu_{L}+\nu_{L}^{C}$ e $w=\nu_{R}+\nu_{R}^{C}$.

Para uma lagrangeana com termos de massa de Dirac e Majorana

$$
\begin{aligned}
-\mathcal{L}_{D M}= & D \bar{\nu}_{L} \nu_{R}+A \bar{\nu}_{L}^{C} \nu_{L}+B \bar{\nu}_{R}^{C} \nu_{R}+\text { h.c. } \\
& =\frac{1}{2} D(\bar{\nu} w+\bar{w} \chi)+A \bar{\chi} \chi+B \bar{w} w \\
& =(\bar{\chi}, \bar{w})\left(\begin{array}{cc}
A & \frac{1}{2} D \\
\frac{1}{2} D & B
\end{array}\right)\left(\begin{array}{c}
\chi \\
w
\end{array}\right)
\end{aligned}
$$

quando diagonalizada nos fornece dois autovalores de massa

$$
m_{1,2}=\frac{1}{2}(A+B) \pm \sqrt{(A-B)^{2}+D^{2}}
$$

que correspondem aos autoestados de massa de Majorana

$$
\begin{aligned}
& \eta_{1}=\cos \theta \chi-\operatorname{sen} \theta w \\
& \eta_{2}=\operatorname{sen} \theta \chi+\cos \theta w
\end{aligned}
$$

com

$$
\tan 2 \theta=D /(A-B)
$$

Vemos assim que no caso mais geral, a Eq. (1.11) descreve duas partículas de Majorana com massas distintas. É possivel mostrar que um férmion de Dirac corresponde, no limite do caso geral, a duas partículas de Majorana. Contudo, todos os férmions, com exceção dos neutrinos, são forçados a esse limite devido a conservação da carga elétrica.

\section{Mecanismo see-saw}

Podemos considerar o modelo mais simples de introduzir massa para os neutrinos exatamente da mesma forma que para os demais férmions. Para isto, devemos adicionar singletos de mão direita, um para cada espécie de neutrino $\nu_{l R}$, ao MP

$$
\mathcal{L}=-f_{l l^{\prime}} \bar{L}_{l L} \widetilde{\phi} \nu_{l^{\prime} R}+\text { h.c. },
$$

onde $\widetilde{\phi}=i \sigma_{2} \phi^{*}$ e $\phi$ é o dubleto de Higgs que aparece no MP, $L_{l L}^{T}=\left(\nu_{l}, l_{l}\right)_{L}$ o dubleto dos férmions de mão esquerda e os índices $l$ e $l^{\prime}$ representam a família. Quando o campo de Higgs adquire o valor esperado do vácuo $\left\langle\phi^{0}\right\rangle=\frac{v_{D}}{\sqrt{2}}$, aparece o termo de massa 


$$
\mathcal{L}=-f_{l l^{\prime}} \frac{v_{D}}{\sqrt{2}} \bar{\nu}_{l L} \nu_{l^{\prime} R}+\text { h.c.. }
$$

Nessa base de autoestados do sabor, os elementos da matriz de massa ainda não diagonalizada são:

$$
M_{l l^{\prime}}=\frac{v_{D}}{\sqrt{2}} f_{l l^{\prime}}
$$

Para obtermos autoestados de massa, é feita uma diagonalização dessa matriz através de uma transformação biunitária, $U^{\dagger} M V=m$, sendo $m$ agora uma matriz diagonal.

Este modelo, contudo, apresenta vários problemas. Primeiramente, a matriz cujos elementos são $f_{l l^{\prime}}$ é completamente arbitrária, uma vez que seus parâmetros não podem ser experimentalmente determinados. Também não obtemos resposta para o fato dos neutrinos serem tão leves comparados aos demais férmions. Se os acoplamentos $f_{l l^{\prime}}$ são muito pequenos com relação aos acoplamentos equivalentes para os férmions carregados, conseguiremos tal resposta. Porém, não existe uma razão para que tal fato aconteça.

Vimos, contudo, que podemos generalizar uma partícula de Dirac em duas de Majorana. Baseados nessa idéia podemos aventar uma razão para a pequena massa dos neutrinos. Para ver isto, analisemos o caso de uma geração. O termo de massa de Dirac aparece do acoplamento com $\phi$ e é natural assumir que seja da mesma ordem de magnitude que o termo de massa dos férmions carregados da mesma geração. Se na Eq. (1.12) supomos $B \gg D$ e $A$ nulo (pois vamos trabalhar apenas com neutrinos de mão esquerda), os autovalores ficam

$$
\begin{array}{r}
m_{1} \simeq \frac{D^{2}}{B}, \\
m_{2} \simeq B .
\end{array}
$$

Dessa forma teremos $m_{1} \ll D$, o que explicaria porque os neutrinos são muito mais leves que os férmions carregados. Este mecanismo é conhecido como Mecanismo see-saw [11].

Dados argumentos cosmológicos atuais, a massa total dos neutrinos se restringe a no máximo aproximadamente $1 \mathrm{eV}$ (WMAP) [12]. Isso implica que

$$
B>3 \times 10^{13} \mathrm{GeV} .
$$

Essa escala de massa é muito maior que a escala eletrofraca, que é da ordem de $10^{2} \mathrm{GeV}$, o que parece indicar que a pequenez da massa dos neutrinos seria um efeito de física nova à baixa energia.

\subsubsection{Duplo decaimento beta}

O duplo decaimento beta corresponde a uma transição rara entre dois núcleos com a mesma massa A e um aumento de $\mathrm{Z}$ em duas unidades. Este processo poderá ocorrer apenas se o núcleo 
inicial for menos energético que o final e os dois sejam mais energéticos que o núcleo intermediário. Tipicamente estas condições poderão ocorrer apenas do estado fundamental do núcleo inicial para o estado fundamental do final, contudo, o decaimento para estados excitados poderá ocorrer em alguns casos energeticamente possíveis.

O duplo decaimento envolvendo dois neutrinos, $\beta \beta 2 \nu$

$$
(Z, A) \rightarrow(Z+2, A)+e_{1}^{-}+e_{2}^{-}+\bar{\nu}_{e 1}+\bar{\nu}_{e 2},
$$

conserva não apenas a carga elétrica como também o número leptônico. No nível fundamental (quarks), estas são transições de dois quarks d em dois quarks u ou vice-versa. São, portanto, processos de segunda ordem em interações fracas com taxas de decaimento muito baixas: os núcleos possuem uma vida média típica $T$ com relação a esse decaimento de $T>10^{19}$ anos.

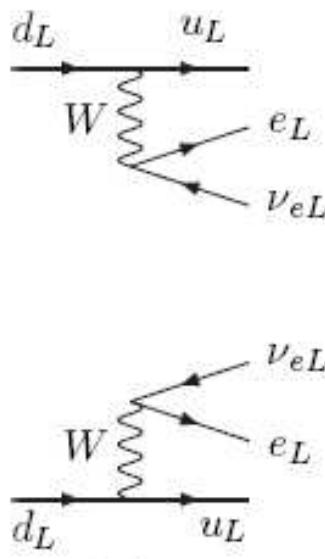

(a)

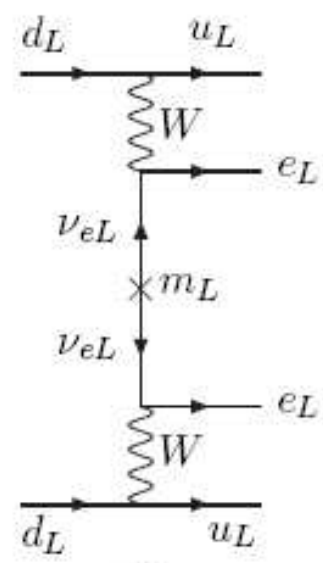

(b)

Figura 1.1: Duplo decaimento beta (a) com e (b) sem neutrinos, respectivamente.

Entretanto, há uma possibilidade teórica da existência do duplo decaimento beta sem neutrinos, $\beta \beta 0 \nu$

$$
(Z, A) \rightarrow(Z+2, A)+e_{1}^{-}+e_{2}^{-},
$$

que viola número leptônico em duas unidades. Neste caso, o neutrino (antineutrino) do elétron produzido em um dos decaimentos beta fundamentais será absorvido no outro com quiralidade invertida (Fig. 1.1). Desta forma, o $\beta \beta 0 \nu$ também quebra a conservação de quiralidade, sendo, portanto, possível apenas se neutrinos tiverem massa de Majorana não nula.

Podemos mostrar que, se o $\beta \beta 0 \nu$ for observado, será possível a obtenção de um valor apropriado para a massa típica do neutrino $\left\langle m_{\nu}\right\rangle$. Consideraremos aqui que a troca de neutrinos de Majorana seja o único mecanismo responsável pelo decaimento.

A taxa de decaimento diferencial do processo $\beta \beta 0 \nu$ é dada por 


$$
d \Gamma=2 \pi \sum_{\text {spin }}|M|^{2} \delta\left(E_{1}+E_{2}+E_{f}-M_{i}\right) \frac{d p_{1}}{(2 \pi)^{3}} \frac{d p_{2}}{(2 \pi)^{3}},
$$

sendo $E_{1(2)}$ e $p_{1(2)}$ as energias totais e os momentos dos elétrons, $E_{f}\left(M_{i}\right)$ a energia final (massa final) do estado nuclear e $M$ a amplitude de transição.

A parte leptônica da amplitude, envolvendo a emissão e reabsorção do neutrino de Majorana pode ser escrita como

$$
M^{(l)}=\frac{m_{\nu}}{q^{2}-m_{\nu}^{2}}\left[\bar{u}_{e}\left(p_{1}\right) \gamma_{\mu} P_{L} \gamma_{\lambda} v_{e}\left(p_{2}\right)\right]
$$

Assim, a amplitude de transição para um neutrino de Majorana de massa $m_{\nu}$ é simplesmente o produto de $m_{\nu}$ e a combinação dos elementos de matriz nucleares. Desta forma, se em cada um dos vértices um elétron é emitido, $U_{e \nu}$ aparece em cada um e a amplitude da reação conterá o fator $U_{e \nu}^{2}$

$$
\left\langle m_{\nu}\right\rangle=\sum_{\alpha} U_{e \alpha}^{2} m_{\alpha}
$$

Se um limite superior para a taxa de decaimento for experimentalmente estabelecido, será possível deduzir um limite superior para $\left\langle m_{\nu}\right\rangle$. Contudo, se $\beta \beta 0 \nu$ for observado, poderá ser calculado o valor de $\left\langle m_{\nu}\right\rangle$.

Experimentalmente, o que é medido é o tempo médio de vida do decaimento [13]. Assumindo que o processo ocorra através da troca de neutrinos leves $\left(m_{\nu}<10 \mathrm{MeV}\right)$, o tempo de vida médio teórico é dado por:

$$
\left[T_{1 / 2}^{0 \nu}\right]^{-1}=G^{0 \nu}\left|M_{0 \nu}\right|^{2}\left(\frac{\left\langle m_{\nu}\right\rangle}{m_{e}}\right)^{2}
$$

sendo $G^{0 \nu}$ a integral no espaço de fase e $m_{e}$ a massa do elétron.

O melhor resultado encontrado para $\beta \beta 0 \nu$ foi obtido pelo grupo de Heidelberg-Moscow [14] que usou $11 \mathrm{~kg}$ de Ge enriquecido. Eles encontraram um sinal que forneceu um limite na meia-vida de $T_{1 / 2}^{0 \nu}>1,9 \times 10^{25}$ anos (90\% CL.). Isto implica uma massa $m_{e}<0,26$ (0,34\% (90\%) CL.) Além do resultado de Heidelberg-Moscow, nenhum outro experimento forneceu resultado positivo.

No decaimento $\beta \beta 0 \nu$, o sinal experimental corresponde a 2 elétrons cujas energias se somam ao $\mathrm{Q}$ valor da transição nuclear, enquanto que para $\beta \beta 2 \nu$ o espectro de energia dos dois elétrons será contínuo, pois parte do Q valor é levado com os neutrinos. Apesar da dependência de $\mathrm{Q}$ ser bem diferente para as taxas de decaimento de $\beta \beta 0 \nu$ e $\beta \beta 2 \nu$, essa dependência é muito fraca para $\beta \beta 0 \nu$, de forma que a sensibilidade será melhor para isótopos com Q de valor alto.

Atualmente, apenas dois experimentos de larga escala estão em atividade. Cuoricino, na Itália, que usa bolômetros correndo a temperaturas muito baixas procurando pelo decaimento de ${ }^{120} \mathrm{Te}$ com um Q-valor de $2530 \mathrm{keV}$. O limite obtido para a meia vida $\mathrm{T}_{1 / 2}^{0 \nu}\left({ }^{120} \mathrm{Te}\right)>2,2 \times 10^{24}$ anos $(90 \% \mathrm{CL})$, 
que implica em um limite superior para a massa de um neutrino de Majorana $\langle m\rangle<0,2-1,1 \mathrm{eV}$ $[15]$.

Outro experimento, NEMO-3 [16] procura pelo duplo decaimento beta sem neutrinos $(\beta \beta 0 \nu)$ através da detecção direta dos dois elétrons. Seu método é baseado na detecção de vestígios de elétrons e suas energias em um calorímetro. O detector tem forma cilíndrica com uma folha de isótopos no centro rodeada pelo calorímetro. Em contraste aos experimentos anteriores com ${ }^{76} \mathrm{Ge}$, ele não detecta apenas a energia total, mas outros parâmetros do processo como: a energia dos elétrons individualmente, o ângulo entre eles e as coordenadas do evento. Nenhuma evidência para o decaimento $\beta \beta 0 \nu$ foi encontrada para os isótopos utilizados [17].

\subsection{Evidências de oscilação}

Neutrinos solares e atmosféricos começaram a ser observados na década de 60 , e foram de importância crucial no estudo de oscilações.

Experimentos que mediam o fluxo de neutrinos atmosféricos detectaram um "desaparecimento" de neutrinos muônicos quando estes se propagavam na ordem de centenas de kilômetros. Já em experimentos com neutrinos solares, a quantidade detectada de neutrinos eletrônicos é inferior a quantidade esperada em praticamente todos os experimentos.

\subsubsection{Neutrinos solares}

A taxa de produção de neutrinos solares, assim como de todas as taxas das reações termonucleares que ocorrem em seu interior são hoje baseadas no Modelo Padrão Solar (MPS) [18]. Estas reações ocorrem via dois canais principais: ciclo CNO e pp. Em ambos os casos a fusão de prótons em hélio ocorre a partir da reação

$$
4 p \rightarrow{ }^{4} \mathrm{He}+2 e^{+}+2 \nu_{e}+\gamma,
$$

que libera uma energia de cerca de $26 \mathrm{MeV}$, sendo parte, aproximadamente $0,59 \mathrm{MeV}$, em forma de neutrinos.

Pelo ciclo pp neutrinos são emitidos em 5 reações diferentes e no ciclo CNO em 3 reações dando origem a distribuições de energia bem conhecidas. Três destas linhas possuem espectro monocromático enquanto as demais, espectro contínuo. 


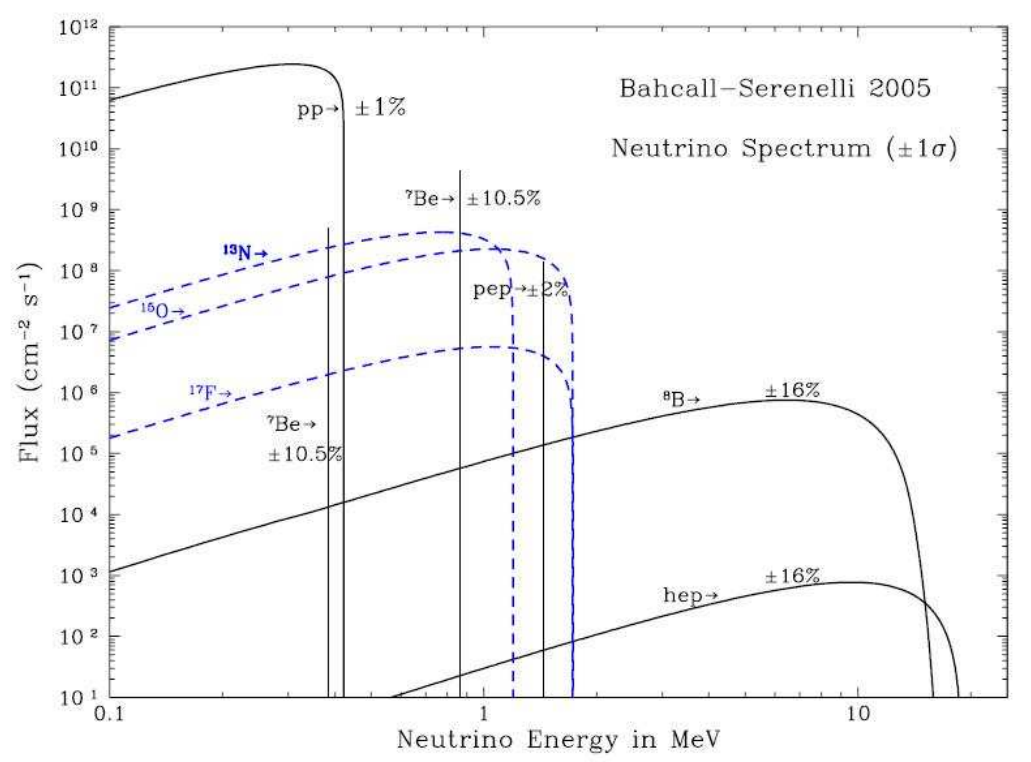

Figura 1.2: Fluxo de neutrinos predito pelo MPS em função da energia.

O resultado da Fig (1.2), baseado no MPS, é descrito a partir das propriedades do Sol e sua evolução até a entrada na cadeia principal. Parâmetros observacionais também são utilizados como a luminosidade da superfície $\left(3,8 \times 10^{26} \mathrm{~W}\right)$, idade $\left(4,5 \times 10^{9}\right.$ anos $)$, raio $\left(6,9 \times 10^{8} \mathrm{~m}\right)$ e massa $\left(1,9 \times 10^{3} \mathrm{~kg}\right)$, além de considerar simetria esférica, equilíbrio hidrostático e térmico, equação de estado de um gás ideal. A figura mostra o espectro de fluxos de neutrinos para cada uma das oito reações preditas pelo modelo.

O fluxo mais abundante de neutrinos (pp) é bem relacionado com a luminosidade do Sol, já bem conhecida, tendo assim uma incerteza pequena $(\approx 1 \%)$. Já o fluxo de neutrinos ${ }^{8} \mathrm{~B}$, mais energéticos, possuem uma incerteza de cerca de $20 \%$ enquanto que o dos neutrinos hep, com maior energia que os demais é bem menos conhecido.

Historicamente, neutrinos solares foram detectados por 8 experimentos: Homestake [19, 20]; Sage, Gallex e GNO [21, 22, 23]; Kamiokande e Super-Kamiokande [24, 25]; SNO [26, 27, 28, 29, 30] e Borexino [31, 32].

O experimento de Homestake capta neutrinos eletrônicos atravéz da reação

$$
\nu_{e}+{ }^{37} \mathrm{Cl} \rightarrow{ }^{37} \mathrm{Ar}+e^{-},
$$

com limiar de energia de 0,814 MeV. Já os experimentos de Sage, Gallex e GNO partem da reação

$$
\nu_{e}+{ }^{71} \mathrm{Ga} \rightarrow{ }^{71} \mathrm{Ge}+e^{-},
$$

que possui limiar de energia de $0,233 \mathrm{MeV}$. 
O experimento Borexino, atualmente tomando dados capta neutrinos de baixa energia através de um líquido cintilador. A reação de espalhamento elástico $\nu_{a}+e^{-} \rightarrow \nu_{a}+e^{-}$(EE) é sensível a todos os sabores de neutrinos pois envolve $\mathrm{CC}$ e $\mathrm{CN}$.

Kamiokande, e o experimento sucessor Super-Kamiokande (SK) detectam elétrons emitidos por espalhamento elástico atravéz de radiação Cherenkov. Este processo é sensível a todos os sabores de neutrinos pois engloba $\mathrm{CN}$ e CC. O limiar de energia do primeiro experimento era de $7,5 \mathrm{MeV}$ e do seu sucessor SK chegou a ser de $5 \mathrm{MeV}$.

Assim como Kamiokande e SK, o detector Sudbury Neutrino Observatory (SNO) também utiliza radiação Cherenkov para captar neutrinos. Este detector foi criado para fornecer uma medida independente do fluxo total de neutrinos solares para então poder entender melhor o fluxo inferior ao esperado de neutrinos eletrônicos.

Nestes oito experimentos foram detectados um déficit de neutrinos eletrônicos com relação ao esperado pelo MPS e, além disto, este déficit foi diferente para cada um dos experimentos. Estas diferenças poderiam ser explicadas tanto por um desconhecimento da verdadeira física solar ou física de neutrinos, quanto por erros experimentais. Contudo, o modelo solar responde bem a outros testes experimentais e com o passar do tempo as suspeitas de problemas com os experimentos foram sendo deixadas de lado pelo fato dos experimentos serem calibrados e utilizarem variadas técnicas de detecção.

Em suma, o problema do neutrino solar não é apenas devido ao déficit no fluxo de neutrinos. Em particular, a dependência com a energia dos resultados dos diversos experimentos indicava que o efeito pudesse ser de origem dinâmica.

\subsubsection{Neutrinos atmosféricos}

Neutrinos atmosféricos são neutrinos e antineutrinos eletrônicos e muônicos produzidos nos chuveiros hadrônicos na atmosfera devido a interação de raios cósmicos com nitrogênio e oxigênio no topo da atmosféra a cerca de $15 \mathrm{~km}$ da Terra. Píons e Kaons produzidos decaem :

$$
\pi^{ \pm}\left(K^{ \pm}\right) \rightarrow \begin{aligned}
& \mu^{ \pm}+\nu_{\mu}\left(\bar{\nu}_{\mu}\right) \\
& \mu^{ \pm} \rightarrow e^{ \pm}+\nu_{e}\left(\bar{\nu}_{e}\right)+\bar{\nu}_{\mu}\left(\nu_{\mu}\right)
\end{aligned}
$$

Partindo das Eqs.(1.30) são esperados grosso modo, a baixa energia, dois neutrinos muônicos para cada neutrino eletrônico. Levando em consideração as diferenças dos tempos de vida de $\pi^{ \pm}$e $K^{ \pm}$ e também reações não dominantes que produzem neutrinos atmosféricos, o cálculo da razão $\nu_{\mu} / \nu_{e}$ dependerá da energia e do ângulo zenital da trajetória dos neutrinos. Assim, teremos uma razão 2 apenas para neutrinos de baixa energia e trajetórias horizontais, e valores maiores para outros casos.

As técnicas de deteç̧ão destes neutrinos serão diferentes conforme a energia. Podem ser observados por interações de $\mathrm{CC}$ dentro do detector, assim como neutrinos e antineutrinos muônicos de maior energia também podem ser detectados indiretamente observando os múons produzidos por CC na rocha nas proximidades do detector.

O cálculo preciso do fluxo de neutrinos atmosféricos possui algumas dificuldades, uma vez que depende do espectro e composição química dos raios cósmicos e produção de $\pi$ 's e K's. As primeiras 
detecções foram em 1960 na África do Sul [33] e na Índia [34]. Eles mediram apenas o fluxo horizontal de neutrinos, pois não podiam discriminar neutrinos vindos diretamente da atmosfera (downgoing) dos que foram produzidos na atmosfera do outro lado do planeta atravessando-o assim para chegar ao detector (upgoing). A razão obtida não estava de acordo com cálculos teóricos, mas o efeito não era estatísticamente significante.

Posteriormente esta razão foi medida por diversos detectores [35]. Em praticamente todos os experimentos a razão encontrada foi menor que a esperada por um fator de 0,6. Esta discrepância é a base da chamada anomalia do neutrino atmosférico. Sendo a razão menor que a esperada, o ponto de observação foi determinar se a causa era um desaparecimento de $\nu_{\mu}$ ou aparecimento de $\nu_{e}$.

A partir dos resultados de Super-Kamiokande em 1998 [36] e posteriormente Soudan 2, Macro $[37,38,39,40,41]$ concluiu-se que os dados de $\nu_{e}$ estão de acordo com o modelo teórico enquanto que os de $\nu_{\mu}$ apresentam um déficit.

\subsubsection{Neutrinos de reatores e aceleradores}

Neutrinos podem ser detectados a partir de feixes provenientes de reatores nucleares. Este tipo de experimento também é chamado de experimento de "desaparecimento" pois devido a baixa energia $\left(E_{\nu} \approx 3 \mathrm{MeV}\right)$ dos neutrinos não é possível detectar neutrinos do múon e do tau. Experimentos serão ou não sensíveis à oscilação dependendo da distância entre fonte e detector como veremos mais adiante.

Muitos experimentos de reatores não encontraram evidências positivas de oscilação: Gosgen [42], Krasnoyarsk [43], Bugey [44], CHOOZ [45], Palo Verde [46]. Em KamLAND veio o primeiro resultado consistente com o déficit esperado com 99,95\% de confiança [47].

Experimentos com aceleradores produzem neutrinos a partir do decaimento de píons produzidos pelo espalhamento de prótons acelerados até um alvo fixo. Assim, são muito semelhantes aos neutrinos atmosféricos devido a natureza do feixe.

Os primeiros experimentos de longa distância entre ponto de produção e ponto de detecção (long baseline) foram K2K e MINOS, com feixes que percorrem respectivamente 235 e $730 \mathrm{~km}$. Seus resultados confirmaram que $\nu_{\mu}$ oscilam de forma compatível com o observado com dados de neutrinos atmosféricos [48, 49].

Este tipo de experimento também pode possuir curta distância entre emissão e detecção dos neutrinos (short baseline). A única evidência de oscilação em experimentos de curta distância aconteceu com o Liquid Scintillator Neutrino Detector (LSND) que favoreceu uma região do espaço de parâmetros nunca observada anteriormente [50]. Esta região foi posteriormente testada e excluída pelos experimentos KARMEN [51] e MiniBooNE [52].

\subsection{Oscilação no vácuo}

Neutrinos são produzidos e detectados através da interação fraca dos autoestados de sabor $\nu_{\alpha}$ $(\alpha=e, \mu, \tau)$. Estes autoestados não são autoestados da matriz de massa e, após produzidos, se 
propagam como a superposição dos autoestados de massa $\nu_{i}(\mathrm{i}=1,2,3)$. Autoestados de sabor e de massa estão relacionados pela matriz unitária de transformação [53]:

$$
\nu_{\alpha}=U_{\alpha i} \nu_{i}
$$

com $\alpha=e, \mu, \tau$ e $i=1,2,3$, sendo

$$
\nu_{\alpha}=\left(\begin{array}{c}
\nu_{e} \\
\nu_{\mu} \\
\nu_{\tau}
\end{array}\right) \text { e } \nu_{i}=\left(\begin{array}{c}
\nu_{1} \\
\nu_{2} \\
\nu_{3}
\end{array}\right)
$$

U corresponde a matriz de Maki-Nakagawa-Sakata (MNS) e pode ser parametrizada em termos dos ângulos de mistura $\theta_{12}, \theta_{23}, \theta_{13}$, e uma fase, $\delta$, que aqui consideraremos nula:

$$
U=\left(\begin{array}{ccc}
c_{12} c_{13} & s_{12} c_{13} & s_{13} \\
-c_{23} s_{12}-s_{23} s_{13} c_{12} & c_{23} c_{12}-s_{23} s_{13} s_{12} & s_{23} c_{13} \\
s_{23} s_{12}-c_{23} s_{13} c_{12} & -s_{23} c_{12}-c_{23} s_{13} s_{12} & c_{23} c_{13}
\end{array}\right),
$$

onde usamos a notação $c_{i j}=\cos \theta_{i j}$ e $s_{i j}=\operatorname{sen} \theta_{i j}$.

A propagação dos autoestados da massa de neutrinos ultra-relativísticos obedecem aproximadamente a equação de Schrödinger [54]:

$$
i \frac{d}{d x} \nu_{i}(x) \approx H_{i} \nu_{i}(x)
$$

em que consideramos o sistema de unidades naturais $(\hbar=\mathrm{c}=1)$, sendo a hamiltoniana na base de autoestados da massa $H_{0}=(1 / 2 E) \operatorname{diag}\left(m_{1}^{2}, m_{2}^{2}, m_{3}^{2}\right)$. Na base do sabor, aplicando a transformação (1.31),

$$
i \frac{d}{d x} \nu_{\alpha}(x)=U H U^{\dagger} \nu_{\alpha}(x)
$$

Usando a aproximação relativística $E_{i} \cong p+\frac{m_{i}^{2}}{2 E}$, a equação de evolução (1.34) leva a

$$
\nu_{\alpha}(t)=\sum_{i} U_{\alpha i} e^{-i \frac{m_{i}^{2}}{2 E} t} \nu_{i}(0)
$$

mostrando que poderá haver uma mudança no sabor ao longo do caminho.

Em notação de Dirac, $\nu(t)=|\nu(t)\rangle$, a probabilidade de conversão de sabor de um neutrino será: 


$$
\begin{aligned}
P_{\alpha \beta} \equiv P\left(\nu_{\alpha} \rightarrow \nu_{\beta}\right) & =\left|\left\langle\nu_{\beta} \mid \nu_{\alpha}(t)\right\rangle\right|^{2} \\
& =\left|\sum U_{\beta j}^{*} U_{\alpha i}\left\langle\nu_{j} \mid \nu_{i}(0)\right\rangle\right|^{2} \\
& =\sum U_{\beta j}^{*} U_{\alpha j} U_{\beta i} U_{\alpha i}^{*} e^{-i \frac{\Delta m_{i j}^{2}}{2 E} t},
\end{aligned}
$$

sendo $\Delta m_{i j}^{2} \equiv m_{i}^{2}-m_{j}^{2}$ as diferenças de massa quadradas. Contudo, é conveniente analisar esta probabilidade em termos dos parâmetros de oscilação. Assumindo oscilação entre dois neutrinos apenas, a matriz U tem a forma:

$$
U=\left(\begin{array}{cc}
\cos \theta & \operatorname{sen} \theta \\
-\operatorname{sen} \theta & \cos \theta
\end{array}\right)
$$

reduzindo a probabilidade de conversão a

$$
P=\operatorname{sen}^{2} 2 \theta \operatorname{sen}^{2}\left(\frac{\Delta m_{i j}^{2}}{4 E} t\right) .
$$

Devido a diferença de massa, a velocidade de fase se modifica

$$
\Delta v_{f} \approx \frac{\Delta m_{i j}^{2}}{2 E}
$$

modificando também a interferência de forma que o processo seja periódico, retornando sempre ao estado original após um certo percurso. O comprimento de oscilação nos fornece esta distância:

$$
l_{\text {osc }}=\frac{2 \pi}{\Delta v_{f}}=\frac{4 \pi E}{\Delta m_{i j}^{2}} .
$$

Aplicando este comprimento de oscilação a probabilidade de transição teremos:

$$
P=\operatorname{sen}^{2} 2 \theta \operatorname{sen}^{2}\left(\pi \frac{L}{l_{\text {osc }}}\right)
$$

sendo $\mathrm{L} \approx \mathrm{t}$ para neutrinos relativísticos.

A partir da definição de probabilidade de transição podemos definir também uma probabilidade de sobrevivência, tal que:

$$
P_{\alpha \alpha} \equiv P\left(\nu_{\alpha} \rightarrow \nu_{\alpha}\right)=1-P
$$

Para que um experimento de oscilação seja sensível a uma diferença de massa, a energia típica do neutrino e a distância do detector a fonte L devem ser tal que: 


$$
E / L \approx \Delta m_{i j}^{2}
$$

ou seja, $\mathrm{L} \sim 1_{\text {osc }}$.

Os valores típicos de $E / L$ para diferentes tipos de fontes de neutrinos e os correspondentes intervalos de $\Delta m_{i j}^{2}$ para os quais eles são mais sensíveis encontram-se na tabela abaixo.

\begin{tabular}{|l|cc|c|c|}
\hline Experiment & & $\mathrm{L}(\mathrm{m})$ & $\mathrm{E}(\mathrm{MeV})$ & $\Delta m^{2}\left(\mathrm{eV}^{2}\right)$ \\
\hline Solar & & $10^{10}$ & 1 & $10^{-10}$ \\
\hline Atmospheric & & $10^{4}-10^{7}$ & $10^{2}-10^{5}$ & $10^{-1}-10^{-4}$ \\
\hline Reactor & SBL & $10^{2}-10^{3}$ & 1 & $10^{-2}-10^{-3}$ \\
& LBL & $10^{4}-10^{5}$ & & $10^{-4}-10^{-5}$ \\
\hline Accelerator & SBL & $10^{2}$ & $10^{3}-10^{4}$ & $>0.1$ \\
& LBL & $10^{5}-10^{6}$ & $10^{4}$ & $10^{-2}-10^{-3}$ \\
\hline
\end{tabular}

Figura 1.3: Valores característicos de L e E para diferentes fontes de neutrinos e os correspondentes intervalos de $\Delta m_{i j}^{2}$, no vácuo.

\subsection{Oscilação na matéria}

A presença de matéria modifica substancialmente a mistura de neutrinos. Primeiramente eles podem ser absorvidos ou espalhados. Contudo, esses processos são proporcionais ao quadrado da constante de Fermi $\left(G_{F}^{2}\right)$, ou seja, são muito pequenos. Neutrinos também podem sofrer espalhamentos elásticos frontais sem a mudança do momento através de um potencial $\mathrm{V}$ proporcional à densidade de número de férmions no meio.

Os três sabores interagem com a matéria por correntes neutras enquanto apenas neutrinos do elétron podem interagir por correntes carregadas na matéria usual.

Para meios isotrópicos, homogêneos e não polarizados com momento total zero [55], as CC levam a um potencial da forma [56]:

$$
V_{e}(x)= \pm \sqrt{2} G_{F} N_{e}(x)= \pm 7,6 \times 10^{-8} Y_{e} \frac{\rho(x)}{10^{14} \mathrm{~g} / \mathrm{cm}^{3}} \mathrm{eV}
$$

sendo $N_{e}(x)$ a densidade de número de elétrons no meio, $\rho$ a densidade de matéria no meio, $Y_{e}=$ $N_{e} / N_{p}+N_{n}$ a densidade de número de elétrons relativa e o sinal $+(-)$ referem-se a neutrinos (antineutrinos).

Já as CN fornecem a mesma contribuição para todos os sabores de neutrinos $\left(V_{a}\right)$. Desta forma a matriz do potencial induzido pela matéria na base de autoestados de sabor será: 


$$
V_{F}(x)=\left(\begin{array}{ccc}
V_{e}+V_{a} & 0 & 0 \\
0 & V_{a} & 0 \\
0 & 0 & V_{a}
\end{array}\right) \rightarrow V_{F}(x)=\left(\begin{array}{ccc}
V_{e} & 0 & 0 \\
0 & 0 & 0 \\
0 & 0 & 0
\end{array}\right)
$$

uma vez que termos proporcionais à identidade na hamiltoneana não mudarão as fórmulas das probabilidades de transição.

Desta forma, a equação de evolução na matéria fica:

$$
i \frac{d}{d x} \nu_{i}(x)=H(x) \nu_{i}(x)=\left[H_{0}+V(x)\right] \nu_{i}(x)
$$

sendo $H_{m}$ a hamiltoneana na matéria, $H_{0}$ no vácuo.

$$
i \frac{d}{d x} \nu_{\alpha}(x)=H^{\alpha}(x) \nu_{\alpha}(x)=\left[U H_{0} U^{\dagger}+V_{F}(x)\right] \nu_{\alpha}(x)
$$

com $H^{\alpha}(x)$ a hamiltoneana da matéria na base de autoestados de sabor.

Para o caso de dois sabores, a Eq. (1.48) fica:

$$
i \frac{d}{d x}\left(\begin{array}{c}
\nu_{e} \\
\nu_{Y}
\end{array}\right)=\left[\left(\begin{array}{cc}
\cos \theta_{0} & \operatorname{sen} \theta_{0} \\
-\operatorname{sen} \theta_{0} & \cos \theta_{0}
\end{array}\right)\left(\begin{array}{cc}
E_{1} & 0 \\
0 & E_{2}
\end{array}\right)\left(\begin{array}{cc}
\cos \theta_{0} & -\operatorname{sen} \theta_{0} \\
\operatorname{sen} \theta_{0} & \cos \theta_{0}
\end{array}\right)+\left(\begin{array}{cc}
V_{e} & 0 \\
0 & 0
\end{array}\right)\right]\left(\begin{array}{c}
\nu_{e} \\
\nu_{Y}
\end{array}\right)
$$

Fazendo a aproximação $E_{i} \cong p+\frac{m_{i}^{2}}{2 E}$, obtemos:

$$
i \frac{d}{d x}\left(\begin{array}{c}
\nu_{e} \\
\nu_{Y}
\end{array}\right)=\frac{\Delta m^{2}}{4 E}\left(\begin{array}{cc}
-\cos 2 \theta_{0}+A(x) & \operatorname{sen} 2 \theta_{0} \\
\operatorname{sen} 2 \theta_{0} & \cos 2 \theta_{0}-A(x)
\end{array}\right)\left(\begin{array}{c}
\nu_{e} \\
\nu_{Y}
\end{array}\right)
$$

sendo $A(x)=\frac{2 E V(x)}{\Delta m^{2}}, \Delta m^{2}=m_{2}^{2}-m_{1}^{2}$.

Agora, a matriz unitária de transformação $U^{M}$ vai depender apenas do ângulo $\theta_{m}$, ou seja, o ângulo de mistura na matéria.

$$
U^{M}=\left(\begin{array}{cc}
\cos \theta_{m} & \operatorname{sen} \theta_{m} \\
-\operatorname{sen} \theta_{m} & \cos \theta_{m}
\end{array}\right)
$$

e usando $H^{\alpha}=U^{M} H^{M}\left(U^{M}\right)^{\dagger}$, a equação de evolução em termos dos novos parâmetros será:

$$
i \frac{d}{d x}\left(\begin{array}{c}
\nu_{e} \\
\nu_{Y}
\end{array}\right)=\frac{\Delta M^{2}}{4 E}\left(\begin{array}{cc}
-\cos 2 \theta_{m} & \operatorname{sen} 2 \theta_{m} \\
\operatorname{sen} 2 \theta_{m} & \cos 2 \theta_{m}
\end{array}\right)\left(\begin{array}{c}
\nu_{e} \\
\nu_{Y}
\end{array}\right)
$$

Comparando (1.50) com (1.52), obtemos a relação:

$$
\tan 2 \theta_{m}=\frac{\operatorname{sen} 2 \theta_{0}}{\cos 2 \theta_{0}-A(x)} .
$$




\subsubsection{Efeito MSW}

Na Eq. (1.53) vemos que dependendo da magnitude e do sinal de A, que depende do sinal de $\mathrm{V}$ e do sinal de $\Delta m^{2}$, o ângulo de mistura na matéria pode variar com relação ao vácuo.

Este resultado mostra que, quando $A(x) \rightarrow \cos 2 \theta_{0}, \theta_{m} \rightarrow \pi / 4$, independentemente do ângulo $\theta_{0}$, que nos leva à seguinte condição:

$$
\sqrt{2} G_{F} N_{e}=\frac{\Delta m^{2}}{2 E} \cos 2 \theta_{0},
$$

chamada condição de ressonância MSW (Mikheev-Smirnov-Wolfenstein) [57, 58], que, quando satisfeita, levará a uma mistura máxima na matéria.

Em geral podemos ter três casos:

- Se $\cos 2 \theta_{0}>>\mathrm{A}(\mathrm{x})$ : Efeitos de matéria possuem pouca influência e a propagação ocorrerá como no vácuo.

- Se $\cos 2 \theta_{0} \approx \mathrm{A}(\mathrm{x}):$ O neutrino passa pela região de ressonância e ocorre mistura devido ao efeito de matéria.

- Se $\cos 2 \theta_{0}<<\mathrm{A}(\mathrm{x})$ : o neutrino pode atravessar a ressonância sem modificações se, para $\Delta m^{2}$ positivo, $\cos 2 \theta_{0}>0\left(\theta<\frac{\pi}{4}\right)$.

\subsubsection{Condição de adiabaticidade}

Em geral, oscilações na matéria em um meio de densidade não uniforme, não fornecem soluções analíticas para a equação de movimento (1.50). Contudo, quando a densidade de matéria varia muito lentamente, pode ser feita uma aproximação com solução exata. Temos que, na matéria:

$$
\left(\begin{array}{c}
\nu_{e} \\
\nu_{Y}
\end{array}\right)=U^{M}\left(\begin{array}{c}
\nu_{1}^{i} \\
\nu_{2}^{i}
\end{array}\right)=\left(\begin{array}{cc}
\cos \theta_{m} & \operatorname{sen} \theta_{m} \\
-\operatorname{sen} \theta_{m} & \cos \theta_{m}
\end{array}\right)\left(\begin{array}{c}
\nu_{1}^{i} \\
\nu_{2}^{i}
\end{array}\right)
$$

Tomando a derivada temporal:

$$
\frac{d}{d t}\left(\begin{array}{c}
\nu_{e} \\
\nu_{Y}
\end{array}\right)=\dot{U}^{M}\left(\begin{array}{c}
\nu_{1}^{i} \\
\nu_{2}^{i}
\end{array}\right)+U^{M}\left(\begin{array}{c}
\dot{\nu}_{1}^{i} \\
\dot{\nu}_{2}^{i}
\end{array}\right)
$$

e usando a equação de evolução na base do sabor (1.50), temos:

$$
i \frac{d}{d t}\left(\begin{array}{c}
\nu_{1}^{i} \\
\nu_{2}^{i}
\end{array}\right)=\left[\frac{1}{2 E}\left(\begin{array}{cc}
\frac{-\Delta m^{2}}{2} & 0 \\
0 & \frac{\Delta m^{2}}{2}
\end{array}\right)-i U^{M \dagger} \frac{d}{d t} U^{M}\right]\left(\begin{array}{c}
\nu_{1}^{i} \\
\nu_{2}^{i}
\end{array}\right) .
$$


Lembrando que $\mathrm{U}$ depende de $\theta_{m} \rightarrow U\left(\theta_{m}\right)$, se a densidade de matéria for praticamente constante (o ângulo de mistura constante), então $\frac{d}{d t} U^{M}=0$ e assim os autoestados de energia serão os autoestados de massa na matéria:

$$
i \frac{d}{d t}\left(\begin{array}{c}
\nu_{1}^{i} \\
\nu_{2}^{i}
\end{array}\right)=\left(\begin{array}{cc}
-\frac{\Delta m^{2}}{4 E} & -i \frac{d}{d t} \theta_{m} \\
i \frac{d}{d t} \theta_{m} & \frac{\Delta m^{2}}{4 E}
\end{array}\right)\left(\begin{array}{c}
\nu_{1}^{i} \\
\nu_{2}^{i}
\end{array}\right) .
$$

Fica claro que os autoestados da massa $\nu_{j}^{i}$ se misturam durante a evolução.

Se $\frac{\Delta m^{2}}{4 E}>>\frac{d}{d t} \theta_{m}$, os autoestados $\nu_{j}^{i}$ se aproximam dos autoestados da energia e praticamente não vão se misturar durante a evolução, ou seja, $\nu_{e}$ e $\nu_{Y}$ vão se misturar adiabaticamente de acordo com o valor da densidade de elétrons no ponto. Enfim, a condição de adiabaticidade é garantida se o parâmetro de adiabaticidade $\gamma$

$$
\gamma=\left|\frac{\Delta m^{2} / 4 E}{d \theta_{m} / d t}\right|
$$

é grande $(>>1)$ no ponto de ressonância. O valor deste parâmetro depende da densidade local, e é menor no ponto de ressonância.

No caso mais geral quando a condição de adiabaticidade não é necessariamente satisfeita, há uma probabilidade de troca entre os autoestados de massa na matéria. Esta probabilidade é chamada de probabilidade de troca de Landau-Zener-Stuckelberg e é calculada utilizando o método de trajetórias complexas de Landau [59].

O resultado final dependerá da natureza da variação de densidade. Se assumimos esta variação como linear, teremos:

$$
P_{L Z S}=\exp (-\pi \gamma / 2)
$$

Diferentes expressões, válidas para variações exponenciais de densidade [60] assim como para regiões com $\gamma<1[61]$ são encontradas na literatura.

\subsection{Oscilação dominante em neutrinos solares}

O problema do neutrino solar pode ser explicado de forma simples pela oscilação de neutrinos eletrônicos em outros neutrinos ativos, $\nu_{\mu}$ ou $\nu_{\tau}$.

O estudo dos dados de neutrinos solares tem como objetivo determinar o intervalo da diferença de massa quadrada e do ângulo de oscilação que regem o déficit observado de $\nu_{e}$. Para isto todas as possíveis fontes de incerteza devem ser levadas em consideração, como o erro do cálculo do fluxo de neutrinos para cada reação no Sol, incerteza na seção de choque para cada processo de interação e mesmo as incertezas relacionadas ao MPS [62]. Em 2001 os resultados da análise incluindo os resultados dos experimentos com $\mathrm{Ga}, \mathrm{Cl}$ e $\mathrm{SK}$ e $\mathrm{SNO}$ apenas para taxas totais, estavam como se apresenta a figura abaixo. 


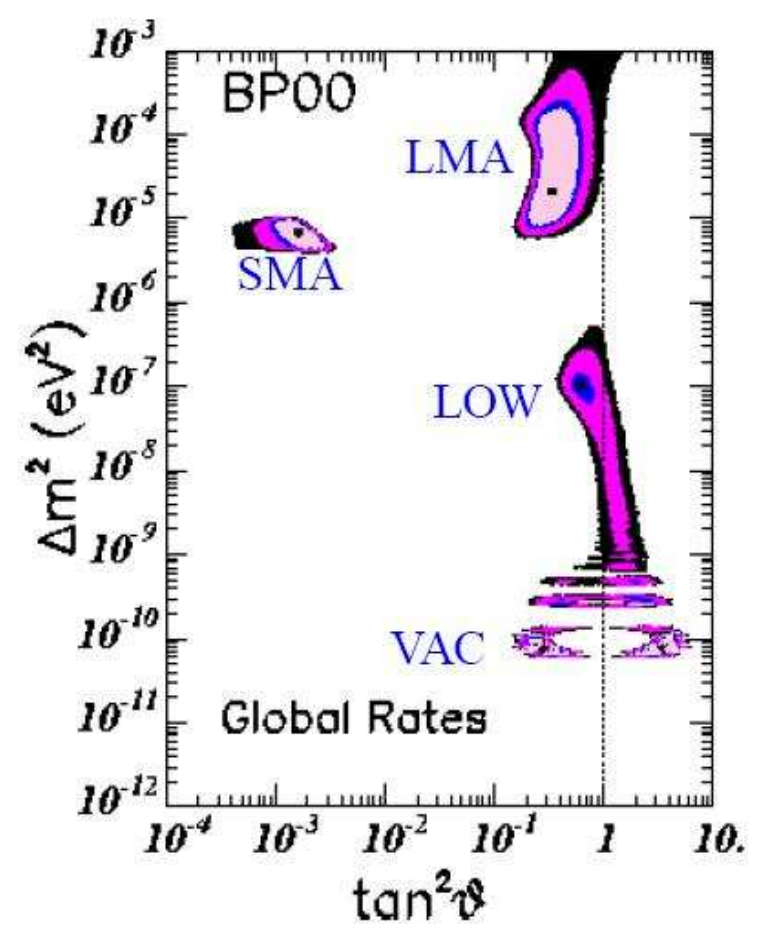

Figura 1.4: Região dos parâmetros de oscilação (com 90\%, 95\%, 99\% e 99,73\% de confiança) compatíveis com a análise das taxas totais dos experimentos com $\mathrm{Cl}$, Ga e dos experimentos de espalhamento SK e SNO [63].

Vemos que existe mais que um regime compatível com os erros experimentais. Estas regiões foram denominadas pelas siglas em inglês: SMA (ângulo de oscilação MSW pequeno), LMA (ângulo de oscilação MSW grande), LOW (pequena massa) e VAC (oscilações no vácuo). A análise da dependência temporal e energética destes diferentes regimes de oscilação foram obtidos dos dados de SK e SNO [63].

A solução de SMA ocorre em regime não adiabático para neutrinos ${ }^{8} \mathrm{~B}$ enquanto que a LMA ocorre em regime adiabático para o mesmo canal, tendo uma probabilidade de sobrevivência maior para neutrinos de menor energia. Na solução LOW a situação é oposta a anterior e para VAC o comprimento de onda de oscilação é da ordem da distância Terra-Sol.

O espectro dia-noite observado não é distorcido com relação ao esperado pelo MPS sob as diversas hipóteses. Assim, uma região do espaço de parâmetros pode ser excluida por prever esta distorção [64]:

- SMA: maiores ângulos não estão de acordo com o espectro de energia, e ângulos menores não fornecem bons ajustes para a razão total;

- VAC: não se ajusta ao espectro observado;

- LMA, LOW: para pequenos $\Delta m^{2}$ a solução é eliminada por prever uma distorção maior que a observada. 
Com a inclusão da dependência energética do fluxo de ${ }^{8} \mathrm{~B}$ em SK e SNO a estes resultados, podemos concluir que a solução que melhor se ajusta ao neutrino solar corresponde a LMA, sendo confirmado pela observação de desaparecimento de $\bar{\nu}_{e}$ em KamLAND. O valor de $\Delta m^{2}$ compatível com essa solução também foi obtida pelo detector KamLAND usando feixes de $\bar{\nu}_{e}$ [65].

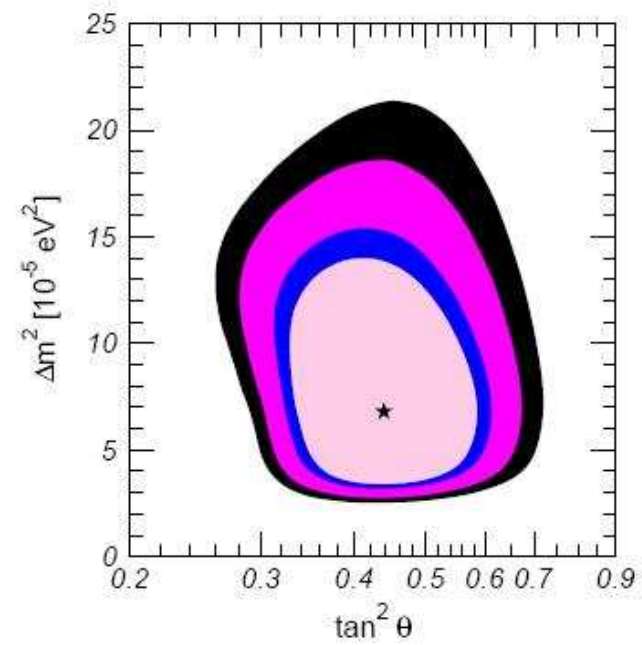

Figura 1.5: Região dos parâmetros de oscilação (com 90\%, 95\% e 99\% de confiança) obtida pela análise global dos dados de neutrinos solares [63].

A Fig.(1.6) mostra que a oscilação de antineutrinos é consistente com parâmetros da solução LMA. Assumindo que a simetria CPT é satisfeita, estes dados combinados com os dos experimentos de neutrinos solares podem gerar regiões compatíveis globais como vemos na Fig.(1.7).

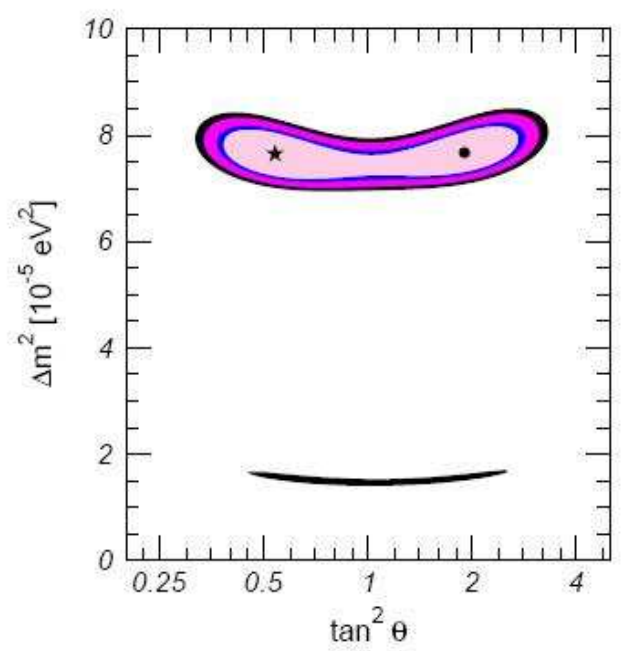

Figura 1.6: Região dos parâmetros de oscilação (com 90\%, 95\% e 99\% de confiança) obtida pela análise dos dados de KamLAND [63]. 


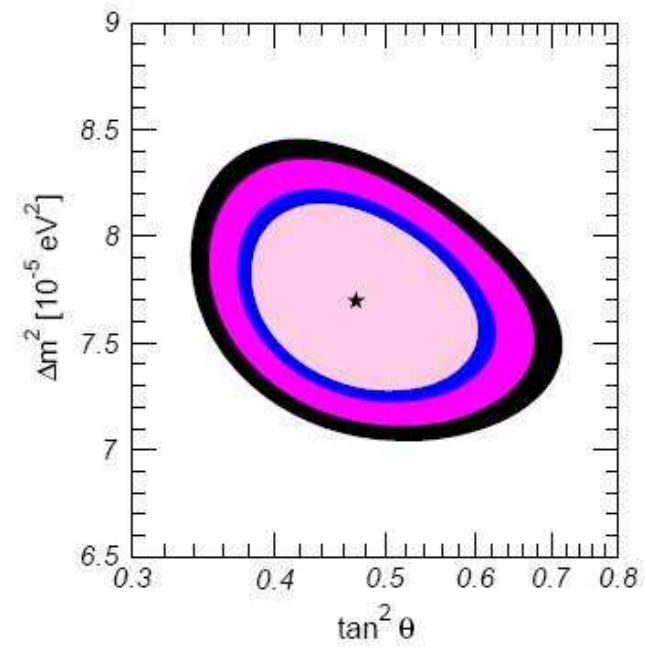

Figura 1.7: Região dos parâmetros de oscilação (com 90\%, 95\% e 99\% de confiança) obtidas pela análise combinada dos dados solares e KamLAND [63].

O melhor resultado atual para diferença de massa solar refere-se aos dados de KamLAND [66] enquanto que para ângulo de oscilaçào solar temos o resultado de SNO [67].

$$
\Delta m_{21}^{2}=7,59_{-0,21}^{+0,21} \cdot 10^{-5} \mathrm{eV}^{2} \mathrm{e} \mathrm{sen}^{2} \theta_{12}=0,32_{-0,04}^{+0,02}
$$

\subsection{Oscilação dominante em neutrinos atmosféricos}

Como vimos, os dados de neutrinos atmosféricos indicam que a oscilação dominante seja a do neutrino muônico. Na Fig. (1.8) vemos a região deteminada pela análise global dos dados atmosféricos. 


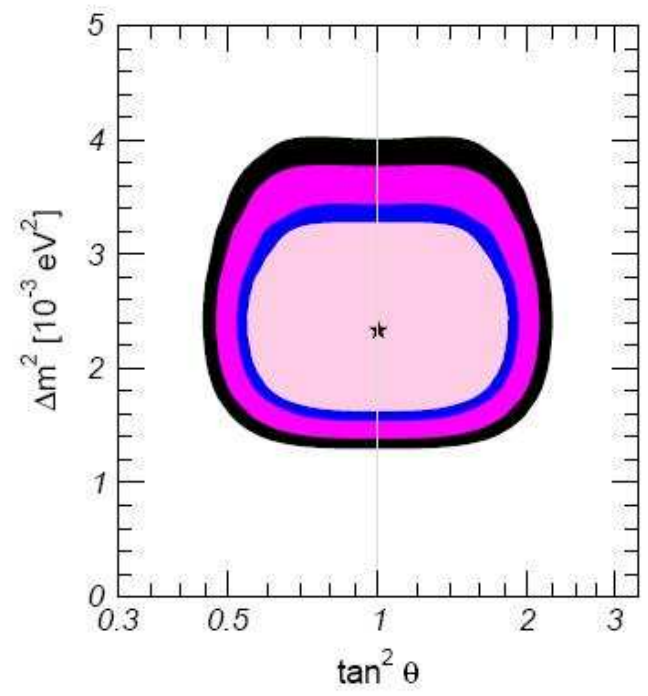

Figura 1.8: Região de parâmetros de oscilação (com 90\%, 95\%, 99\% e 99,73\% de confiança) obtida pela análise dos dados de neutrinos atmosféricos.

O canal de oscilação $\nu_{\mu} \rightarrow \nu_{e}$ já foi descartado. Isto porque os dados de SK para $\nu_{e}$ são muito bem descritos pelo modelo, e também porque se a transição $\nu_{\mu} \rightarrow \nu_{e}$ explicasse os dados atmosféricos, implicaria também na transição $\bar{\nu}_{\mu} \rightarrow \bar{\nu}_{e}$, havendo assim um déficit de $\bar{\nu}_{e}$ no experimento de reator CHOOZ, o que não é observado.

Desta forma, a melhor interpretação para os dados atmosféricos é a oscilação no canal $\nu_{\mu} \rightarrow \nu_{\tau}$, apesar de não observado o aparecimento de $\nu_{\tau}$. Como vimos, o experimento K2K e MINOS confirmam o déficit de neutrinos do múon para distâncias de centenas de kilômetros, assim como esperado para parâmetros atmosféricos. Como vemos na próxima figura, K2K e MINOS determinaram independentemente o $\Delta m^{2}$ relevante e compatível com os resultados atmosféricos. 

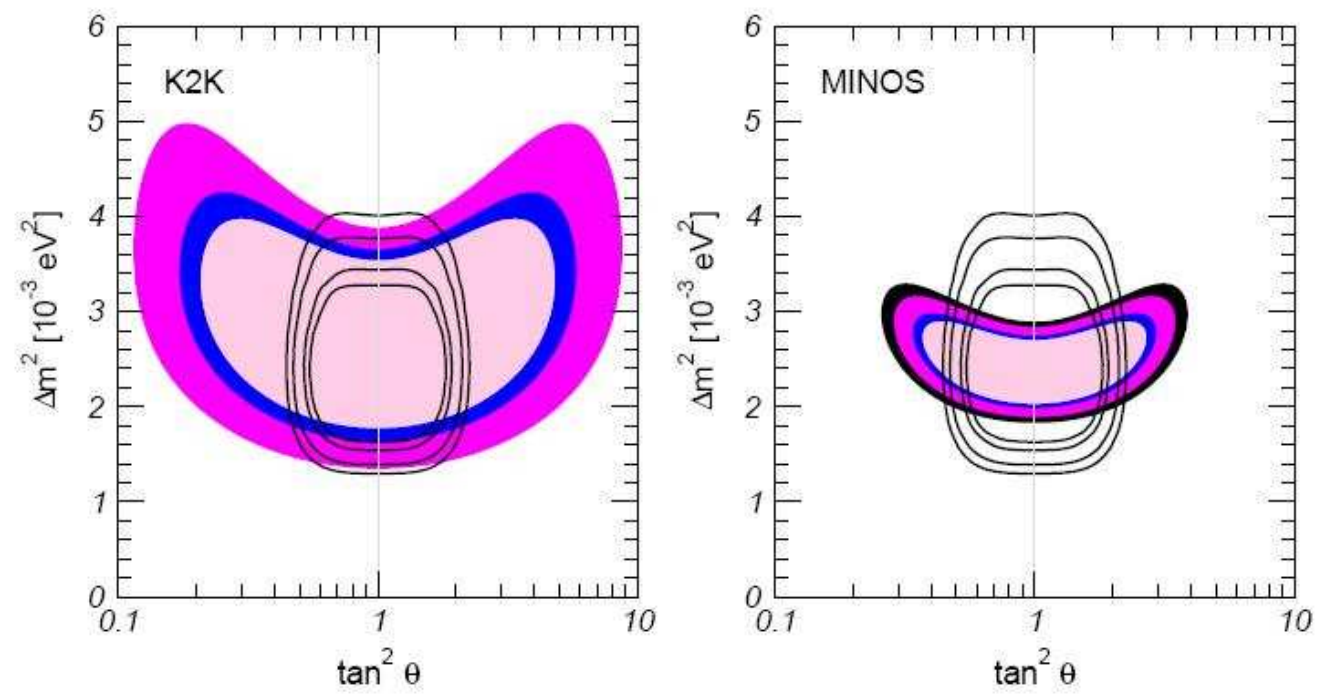

Figura 1.9: Região dos parâmetros de oscilação obtida pela análise dos dados de K2K (esquerda) e MINOS (direita), com 90\%, 95\% e 99\% de confiança. As linhas contínuas não preenchidas sobrepostas correspondem as mesmas regiões para dados atmosféricos [63].

Atualmente, o melhor resultado obtido para a diferença de massa atmosférica é referente ao experimento MINOS [68]. Neste experimento o feixe de neutrinos percorreu a distância de $734 \mathrm{~km}$, com resultado final que segue. Já o angulo de oscilação possui seu melhor resultado a partir da análise dos dados de neutrinos atmosféricos [39, 40].

$\left|\Delta m^{2}\right|=2,74_{-0,26}^{+0,44} \cdot 10^{-3} \mathrm{eV}^{2}$ e $\operatorname{sen}^{2} \theta>0,87$ com $68 \%$ de confiança




\section{Capítulo 2}

\section{Neutrinos de Supernova}

Simulações do colapso do núcleo estelar [69] têm acrescentado bastante ao conhecimento do mecanismo de explosão de supernovas, apesar de uma teoria completa ainda não ter sido desenvolvida. O aspecto da teoria ligado mais diretamente ao nosso estudo diz respeito a emissão de neutrinos durante uma explosão de supernova. Durante a explosão, cerca de $99 \%$ da energia liberada segue na forma de neutrinos, sendo apenas 1 a $2 \%$ liberada como ondas eletromagnéticas. Estes neutrinos carregam em seu espectro a chave da informação, não apenas da natureza do colapso estelar, mas também de propriedades importantes dos neutrinos ainda não exploradas em laboratório.

Veremos neste capítulo o que é conhecido sobre o mecanismo de explosão de supernovas, enfocando o papel que a produção e emissão de neutrinos têm neste mecanismo, assim como os principais resultados de simulações.

\subsection{Mecanismo de explosão de supernovas}

Supernovas (SN) são explosões que marcam o fim da evolução de algumas estrelas, podendo atingir uma luminosidade de $10^{10} \mathrm{~L} \odot$ (luminosidade solar) em poucas semanas. Possuem massa que varia tipicamente de 1 - $10 \mathrm{M}_{\odot}$ (massa solar).

Existem dois tipos principais de supernovas de acordo com o proposto em 1941 por Rudolph Leo Bernhard Minkowski [70]: as SN do tipo I, que não apresentam linhas de emissão e absorção de hidrogênio no espectro e as SN do tipo II, que apresentam linhas de emissão e absorção de hidrogênio no espectro, alargadas pela alta velocidade de ejeção do gás. Em galáxias espirais ocorrem aproximadamente uma SN tipo I a cada 100 anos e uma SN tipo II a cada 30 anos.

As SN tipo II ocorrem por implosão do núcleo de estrelas massivas e são observadas somente nos braços de galáxias espirais e em galáxias irregulares (regiões de formação estelar) e são um pouco menos luminosas que as SN tipo I. Estas últimas ocorrem tanto em galáxias espirais quanto elípticas. Algumas SN do tipo I foram descobertas em braços espirais, recebendo a denominação de tipo Ib, oriundas da queima explosiva do carbono ou colapso do núcleo de estrelas pobres em hidrogênio, enquanto as do tipo I clássicas são chamadas do tipo Ia, ocorrendo em sistemas binários. Nestes sistemas, a SN acontece quando uma estrela anã-branca com massa próxima a massa de 
Chandrasekhar $\left(M_{C} \approx 1,0 M_{\odot}\left(Y_{e} / 0,4\right)^{2}\right)$ [71] recebe massa da companheira em taxas tão altas que uma explosão de nova não ocorre [72, 73]. A origem da explosão reside na extrema sensibilidade à temperatura que a queima do carbono possui, o que resulta em uma explosão descontrolada que destroi completamente a anã-branca.

Supernovas do tipo II são de especial importância em nosso estudo devido à grande produção de neutrinos ao longo das reações nucleares que acarretam em sua explosão.

\subsubsection{Supernovas do tipo II}

Estrelas massivas $\left(M \geq 9,10 M_{\odot}\right)$ passam tranquilamente pelos estágios de queima de carbono, oxigênio e silício e, consequentemente, no início do colapso gravitacional seus núcleos são compostos basicamente de elementos pesados do grupo do ferro. A massa de seus núcleos são de aproximadamente $1,4 M_{\odot}$ (massa de Chandrasekhar), independentemente do total da massa estelar quando o colapso inicia. Zonas de convecção causadas pela queima de C, O e Si nos estágios anteriores fazem com que a composição química do núcleo seja homogênea antes do colapso, resultado este importante para a formação posterior de estrelas de nêutrons.

No início do colapso gravitacional a pressão do gás no núcleo é causada basicamente pelo gás de elétrons relativístico que aparece após as primeiras contrações. A explicação física para a instabilidade dinâmica é que a dissociação dos elementos do grupo do ferro remove a energia térmica do gás, reduzindo assim a pressão do gás. Enquanto a densidade do núcleo aumenta, as energias dos elétrons ficam maiores e elétrons são capturados por prótons dentro do núcleo, o que reduz ainda mais a pressão do gás, e o colapso se acentua. Este processo, chamado de fotodesitegração foi proposto em 1957 por William Alfred Fowler e Fred Hoyle [74].

Durante os primeiros estágios do colapso, neutrinos são emitidos pelo processo de captura de elétrons e escapam livremente da estrela. Alguns neutrinos são também emitidos pela aniquilação de elétrons e pósitrons e por reações do tipo URCA ${ }^{1}$. Com o aumento da densidade do núcleo $(\rho)$, a opacidade ao neutrino também aumenta, até que seu livre caminho médio se torne menor que o raio do núcleo. A energia dos neutrinos é tipicamente maior que a massa de repouso dos elétrons causando uma mudança significativa em seu espectro energético. Esta "armadilha" para os neutrinos ocorre a $\rho>3 \times 10^{11} \mathrm{~g} / \mathrm{cm}^{3}$, formando uma região conhecida como neutrinosfera. A partir daí, a mudança térmica dos neutrinos ocorre devido ao espalhamento com elétrons e processos de emissão e absorção.

Quando a densidade do núcleo fica acima da densidade nuclear, $\rho>2,8 \times 10^{14} \mathrm{~g} / \mathrm{cm}^{3}$, ocorre o colapso, fazendo com que o núcleo pulse e cause uma onda de choque. É nesta fase que a maior parte da energia da SN é liberada através dos neutrinos capturados pelo núcleo massivo. Essa energia é dada pela energia da estrela menos a energia da estrela de neutrons remanescente:

$$
\Delta E=\left[-\frac{G_{N} M^{2}}{R}\right]_{E}-\left[-\frac{G_{N} M^{2}}{R}\right]_{E N},
$$

\footnotetext{
${ }^{1} \mathrm{O}$ processo URCA de emissão de neutrinos consiste de uma captura de elétron seguida de um decaimento $\beta$ [75]:
}

$$
\begin{gathered}
e^{-}+(Z, A) \rightarrow(Z-1, A)+\nu_{e} \\
(Z-1, A) \rightarrow(Z, A)+e^{-}+\bar{\nu}_{e} .
\end{gathered}
$$


sendo $R_{E}$ aproximandamente $10^{10} \mathrm{~cm}$ e $R_{E N}$ alguns quilômetros, teremos $\Delta E \sim 10^{53} \mathrm{erg}$.

A idéia básica do transporte de neutrinos em supernovas foi sugerida nos anos 40 [76], mas foi aceita somente após a descoberta das correntes neutras fracas e sugestão de que a seção de choque de neutrinos em elementos pesados varia com $\mathrm{A}^{2}$, sendo $\mathrm{A}$ o número de nucleons dentro de um núcleo.

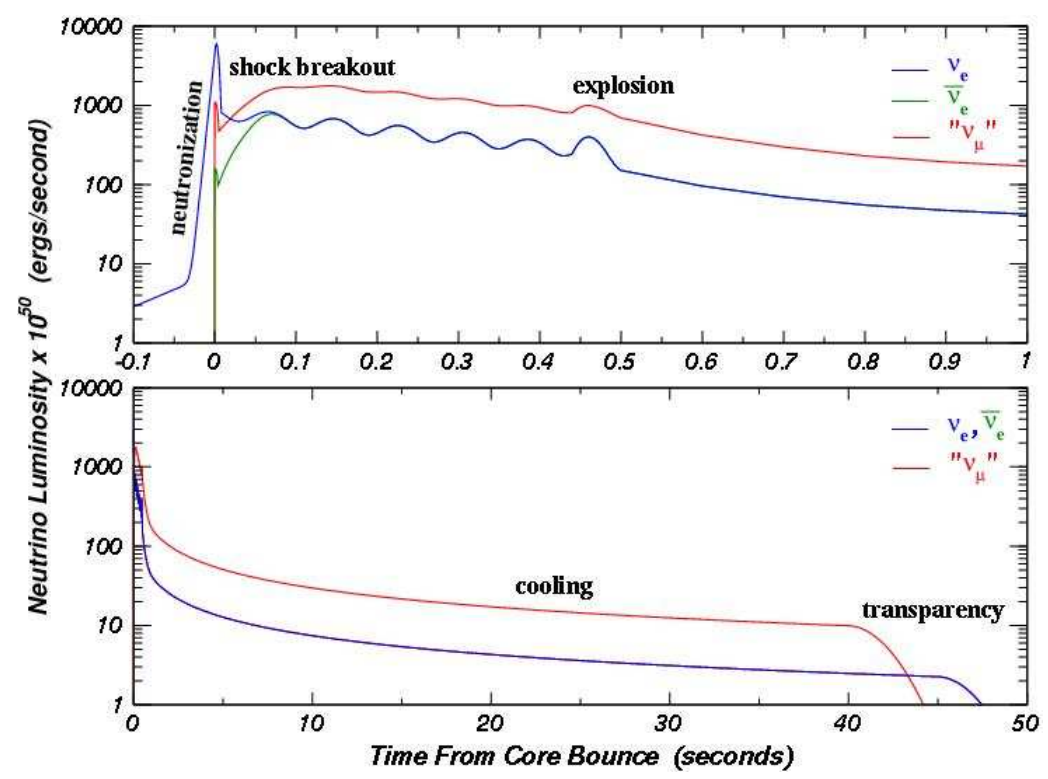

Figura 2.1: Luminosidade de neutrinos instantes após a explosão de uma supernova do tipo II. " $\nu_{\mu}$ " refere-se a (anti)neutrinos que não sejam do elétron [77].

Diferentes simulações da emissão de neutrinos durante a explosão de uma supernova levam a sinais com propriedades diversas. Contudo, existem alguns fatores que são comuns a todas, tais como:

- As luminosidades e fluxos evoluem no tempo de uma maneira razoavelmente bem conhecida. Nos primeiros milisegundos, o sinal esperado é dominado por neutrinos do elétron (pico de neutralização, veja Fig. (2.1), que são produzidos pela captura de elétrons por prótons e núcleos durante a passagem da onda de choque. Juntamente, há um aumento dos neutrinos de todos os sabores até se estabilizarem, iniciando assim um decaimento lento.

- O núcleo de uma supernova corresponde ao único local no universo atual em que pode-se considerar os neutrinos em equilíbrio térmico. Desta forma, esperamos a emissão de um espectro quasi-térmico de neutrinos.

\subsection{Evolução do fluxo de neutrinos}

A emissão temporal de neutrinos por uma supernova corresponde a característica melhor conhecida e com melhores resultados em simulações. Esta emissão é bem marcada por três fases distintas de evolução, sendo todas relacionadas ao processo físico do colapso do núcleo da estrela. 
No momento do colapso em que o núcleo atinge densidade maior que a nuclear, uma onda de choque hidrodinâmica se forma em direção à superfície da estrela. Após alguns milisegundos, a onda de choque atinge a região do núcleo em que os neutrinos estavam presos, liberando-os. Esta onda de choque aciona a emissão de neutrinos durante sua passagem pela neutrinosfera em menos de $1 \mathrm{~ms}$ [77], fase esta com o fluxo máximo de neutrinos, conhecida como breakout, ou erupção do choque.

Com o início da emissão de neutrinos, juntamente com a dissociação nuclear, há um maior consumo de energia da onda de choque, tornando-a uma frente de acreção quase estacionária antes mesmo de deixar o núcleo de ferro. Desta forma, a estrela de nêutrons em formação passa a emitir seu calor de forma constante através de neutrinos. Além disto, o material dissociado vindo juntamente com o choque estagnado produz neutrinos eletrônicos $\left(\nu_{e}\right)$ pela captura de elétrons em prótons. O material quente na frente de choque produz pares elétron-pósitron, e a captura de pósitrons por nêutrons gera um fluxo adicional de antineutrinos eletônicos $\left(\bar{\nu}_{e}\right)$. Esta fase de acreção e resfriamento do manto (accretion) é caracterizada pelo decaimento vagaroso do fluxo de neutrinos, até que uma nova explosão se inicia e a onda de choque renasce.

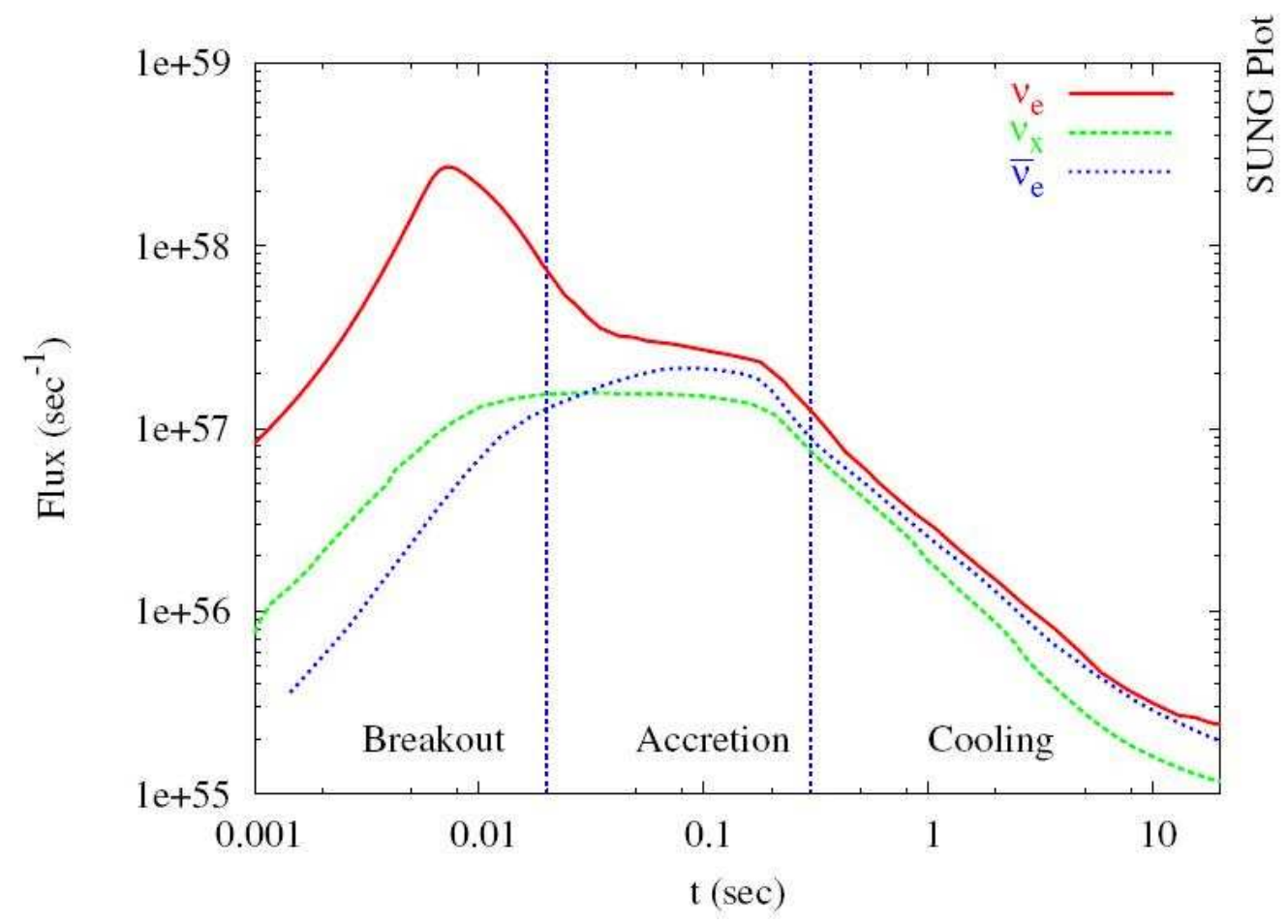

Figura 2.2: Três fases principais da evolução do fluxo de neutrinos: erupção do choque (crescimento rápido), acreção e resfriamento do manto [78].

Após a revitalização da onda de choque, a supernova inicia processos mais estáveis. Uma lei de potência decrescente rege o fluxo de neutrinos e a emissão de uma fração importante da energia de ligação da estrela de nêutrons. Esta fase de resfriamento (cooling) dura entre dezenas de segundos até minutos e termina quando o material da estrela de nêutrons torna-se transparente aos neutrinos [79]. 


\subsection{Espectro de neutrinos}

Neutrinos aprisionados no interior do núcleo da supernova são mantidos em equilíbrio térmico com o plasma através de espalhamento inelástico. Desta forma, é esperada a emissão de um espectro quasi-térmico a uma temperatura próxima à temperatura do plasma onde os neutrinos se desacoplam energeticamente. Contudo, a análise mais refinada do espectro no estágio de resfriamento mostra que este não é exatamente térmico. Uma vez que a seção de choque dos neutrinos aumenta com a energia, o raio da neutrinosfera também aumentará. Desta forma, mesmo se os neutrinos estiverem em equilíbrio térmico com a matéria, haverá uma distorção não-térmica. Veremos a seguir as principais características do espectro de neutrinos [80].

\subsubsection{Neutrinos e antineutrinos do elétron}

Devido a presença de elétrons e pósitrons, $\nu_{e}$ e $\bar{\nu}_{e}$ podem sofrer reações de CC (processos $\beta$, $e+p \leftrightarrow n+\nu_{e}$ e $e^{+}+n \leftrightarrow p+\bar{\nu}_{e}$ ) que estão praticamente ausentes para os outros sabores. Os processos $\beta$ dominam o transporte de $\nu_{e}$ e $\bar{\nu}_{e}$ no interior do núcleo da SN por ter seção de choque muito maior que os outros processos de espalhamento. A contínua criação e captura de neutrinos nestes processos mantem $\nu_{e}$ e $\bar{\nu}_{e}$ em um equilíbrio térmico local com o meio.

Sendo as seções de choque destes processos dependentes da energia, o raio da neutrinosfera e a temperatura característica de $\nu_{e}$ e $\bar{\nu}_{e}$ serão diferentes para neutrinos de diferentes energias: neutrinos mais energéticos se afastarão até raios maiores com menores temperaturas e neutrinos menos energéticos se manterão em raios menores com maior temperatura. Desta forma, a distribuição de energia não será exatamente térmica. O espectro de neutrinos será reduzido para altas e baixas energias devido a diferença de temperatura de onde os neutrinos são emitidos (distorção espectral ou pinching).

\subsubsection{Neutrinos não eletrônicos}

Para neutrinos não eletrônicos $\left(\nu_{x}\right)^{2}$, apenas as reações de CN participam do transporte. No interior do núcleo, $\nu_{x}$ são criados e aniquilados por três processos principais

- Bremsstrahlung nuclear: $N+N \leftrightarrow N+N+\nu+\nu$,

- Aniquilação elétron-pósitron: $e+e^{+} \leftrightarrow \nu_{x}+\nu_{x}$,

- Aniquilação neutrino-antineutrino: $\nu_{e}+\bar{\nu}_{e} \leftrightarrow \nu_{x}+\nu_{x}$,

mantendo-os em equilíbrio térmico local. Para um determinado raio, os processos de criação e aniquilação cessam, mantendo o número de neutrinos na região chamada "esfera de número". Fora da esfera de número, neutrinos ainda sofrem troca de energia com $e^{-}, e^{+}$e nucleons até que a temperatura abaixa e os neutrinos desacoplam energeticamente do meio circundante, local conhecido como "esfera de energia".

\footnotetext{
${ }^{2}$ Designamos genericamente por $\nu_{x}: \nu_{\mu}, \bar{\nu}_{\mu}, \nu_{\tau}, \bar{\nu}_{\tau}$
} 
$\mathrm{Na}$ determinação da distribuição de energia final de $\nu_{x}$ poderia-se imaginar que, pelo fato de o contato térmico acontecer apenas dentro da esfera de energia, a energia média do neutrino seria relacionada com a temperatura do plasma nesta camada. Contudo, o espalhamento do nucleon fora da esfera de número atua como filtro [81], abaixando a energia média para 50-60\% do seu valor.

\subsubsection{Distorção espectral (pinching)}

A natureza quase térmica do espectro do neutrino é uma caracterísica particular da emissão de neutrinos por supernovas.

Uma forma comum de ajustar o espectro de neutrinos de SN é usar um parâmetro de degenerescência efetivo $\eta_{i}$ na distribuição de Fermi-Dirac. Este parâmetro entra na distribuição como um potencial químico efetivo, a uma temperatura efetiva $T_{i}$ para cada espécie de neutrino $i$.

$$
F_{i}^{0}(E) \propto \frac{E^{2}}{\exp \left(E / T_{i}-\eta_{i}\right)+1}, i=e\left(=\nu_{e}\right), \bar{e}\left(=\bar{\nu}_{e}\right), x\left(=\nu_{x}\right)
$$

Os valores de $\eta_{i}$ são os mesmos para qualquer que seja $\nu_{x}$ e são em geral diferentes para $\nu_{e}$ e $\bar{\nu}_{e}$, e não precisam ser constantes ao longo da fase de resfriamento. Tipicamente:

$$
\begin{array}{ccc}
T_{e} \approx(3-4) \mathrm{MeV} & T_{\bar{e}} \approx(5-6) \mathrm{MeV} & T_{x} \approx(7-9) \mathrm{MeV} \\
\eta_{e} \approx 3-5 & \eta_{\bar{e}} \approx 2.0-2.5 & \eta_{x} \approx 0-2
\end{array}
$$

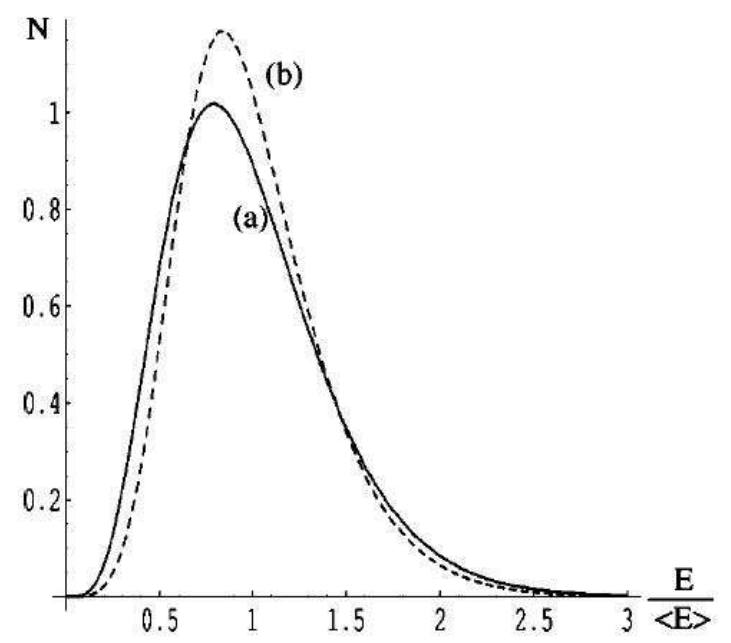

Figura 2.3: Distribuição do número $\mathrm{N}$ de eventos de $\mathrm{CC}$ para (a) um espectro térmico $(\mathrm{T}=3 \mathrm{MeV}$, $\eta=0)$, e (b) um espectro distorcido (pinched) $(\mathrm{T}=3 \mathrm{MeV}, \eta=3)$ [82].

O efeito de distorção não térmica também pode ser quantificado utilizando momentos de ordem superior da distribuição de energia. A introdução de um parâmetro de pinching [81] definido em termos da razão 


$$
p \equiv \frac{1}{a} \frac{\left\langle E^{2}\right\rangle}{\langle E\rangle}
$$

onde $a \simeq 1,3029$ é um peso constante igual a razão do segundo e primeiro momentos da distribuição de Fermi-Dirac com potencial químico zero. Na ausência de pinching, $p=1$. Um espectro com pinching será caracterizado por $p<1$. Já o aumento do espectro nas caudas corresponde a $p>1$.

\subsection{Hierarquia das energias médias}

Outra característica da emissão de neutrinos por supernovas é a hierarquia existente entre as energias médias dos diferentes sabores. Como visto, as propriedades do espectro de neutrinos de supernovas são determinadas pelo processo de transporte perto da neutrinosfera. No caso mais simples, as energias médias de $\nu_{e}$ e $\bar{\nu}_{e}$ são determinadas pela temperatura do plasma ao redor da neutrinosfera. Apesar da seção de choque nos processos $\beta$ possuirem a mesma dependência energética para $\nu_{e}$ e $\bar{\nu}_{e}$, a assimetria entre prótons e nêutrons no núcleo gera uma diferença na opacidade de neutrinos e antineutrinos. Sendo nêutrons mais abundantes, $\nu_{e}$ 's se desacoplam da matéria em raios maiores que $\bar{\nu}_{e}$ 's, tendo portanto energia média menor, $\left\langle E_{\nu_{e}}^{0}\right\rangle\left\langle\left\langle E_{\bar{\nu}_{e}}^{0}\right\rangle\right.$.

A magnitude desta diferença vai depender da estrutura do núcleo, que vai determinar a posição da neutrinosfera. Em simulações de SN, valores típicos variam de 30\% [83] a 50\% [84, 85] como mostram os quadros (a) e (b) da Fig. 2.4.

Já o transporte de $\nu_{x}$, que é dominado por reações de CN, possui taxas de interações muito menores que processos $\beta$. Assim, $\nu_{x}$ 's se desacoplam da matéria em raios menores, implicando uma energia média maior. Em suma, é observada nas simulações a seguinte hierarquia:

$$
\left\langle E_{\nu_{e}}^{0}\right\rangle<\left\langle E_{\bar{\nu}_{e}}^{0}\right\rangle<\left\langle E_{\nu_{x}}^{0}\right\rangle .
$$



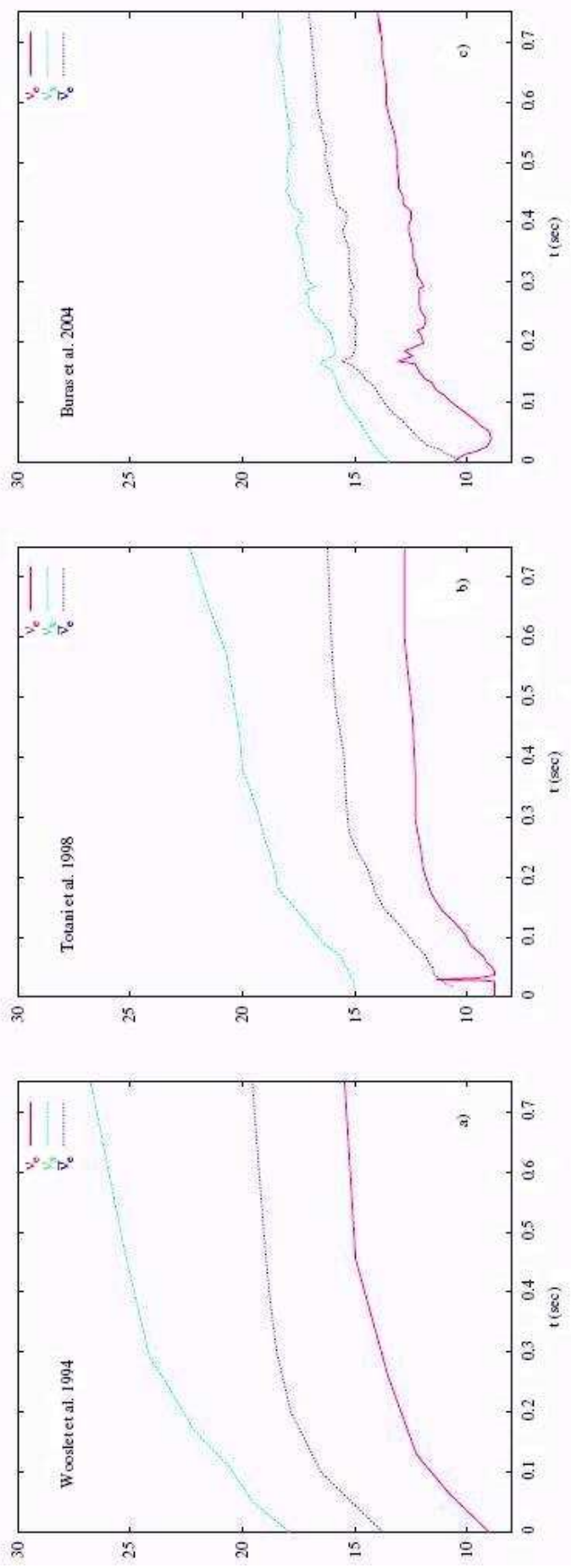

Figura 2.4: Resultados de diferentes simulações para evolução da energia média dos neutrinos [84, 85, 87]. Fica clara a diferença de resultados entre as simulações dos dois primeiros quadros (a) e (b), que utilizam descrição simplificada de $\nu_{x}$ para a do terceiro quadro (c), com descrição mais completa.

A determinação da magnitude entre as energias médias de $\nu_{x}$ e $\bar{\nu}_{e}$ requer mais atenção. Se o 
cálculo for feito a partir da temperatura espectral da esfera de energia, a diferença esperada é grande. Contudo, deve ser levado em consideração o efeito filtro das reações elásticas fora dessa esfera, que faz reduzir a energia média [86]. A diferença final vai depender de detalhes do transporte de $\nu_{x}$ nessas regiões. Simulações consistentes em geral usam descrições simplificadas de $\nu_{x}$ devido a sua baixa relevância na dinâmica da SN.

Simulações recentes de SN hidrodinâmica auto-consistentes $[88,89]$ sugerem que pode estar errada a descrição com grandes diferenças espectrais. Incluindo todas as reações relevantes no transporte de $\nu_{x}$, além de efeitos não considerados em simulações anteriores, a energia média de $\nu_{x}$ difere pouco da de $\bar{\nu}_{e}$, com diferenças não maiores que 20\%, como pode ser visto no quadro (c) da Fig. 2.4.

\subsection{Dinâmica de conversão}

\subsubsection{Regiões de conversão}

A conversão de neutrinos em SN ocorre principalmente nas camadas de ressonância MSW, cujas densidades devem ser (Eq. (1.54))

$$
\rho_{\text {res }} \approx \frac{1}{\sqrt{2} G_{F}} \frac{\Delta m^{2}}{2 E} \frac{m_{N}}{Y_{e}} \cos 2 \theta
$$

sendo $m_{N}$ a massa do nucleon, $Y_{e}$ a fração de elétrons e $E$ a energia do neutrino.

A partir da Eq. (2.7), sabemos que existem duas densidades de ressonância no interior da estrela e, desta forma, os neutrinos que se propagam devem passar por duas ressonâncias MSW:

- Ressonância H: ocorre a altas densidades,

$$
\rho_{\text {res }}^{H} \sim 10^{3}(10 \mathrm{MeV} / \mathrm{E}) \mathrm{g} / \mathrm{cm}^{3},
$$

e é governada pela diferença de massa atmosférica $\Delta m_{a t m}^{2} \equiv \Delta m_{32}^{2}$ e pelo ângulo $\theta_{13}$.

- Ressonância L: ocorre a baixas densidades

$$
\rho_{\text {res }}^{L} \sim(30-140)(10 \mathrm{MeV} / \mathrm{E}) \mathrm{g} / \mathrm{cm}^{3},
$$

sendo determinada pelos parâmetros solares $\Delta m_{\odot}^{2} \equiv \Delta m_{21}^{2}$ e $\theta_{12} \equiv \theta_{\odot}$.

\subsubsection{Espectro de massa}

Como vimos, o sistema de três neutrinos ativos $\nu_{\alpha} \equiv\left(\nu_{e}, \nu_{\mu}, \nu_{\tau}\right)$ se mistura no vácuo da seguinte forma:

$$
\nu_{\alpha}=U \nu_{i}
$$


$\operatorname{com} \nu_{i} \equiv\left(\nu_{1}, \nu_{2}, \nu_{3}\right)$ o vetor de autoestados de massa U a matriz de mistura. Temos que

$$
\Delta m_{32}^{2} \equiv m_{3}^{2}-m_{2}^{2}=\Delta m_{a t m}^{2}
$$

é a escala de diferença de massa que resolve o problema do neutrino atmosférico e que

$$
\Delta m_{21}^{2} \equiv m_{2}^{2}-m_{1}^{2}=\Delta m_{\odot}^{2},
$$

é a escala de diferença de massa que resolve o problema do neutrino solar, e $\Delta m_{\odot}^{2}$ é a solução de grande ângulo de mistura (LMA) para neutrinos solares.

Uma vez que $\nu_{\mu}$ e $\nu_{\tau}$ são indistinguíveis no estudo de neutrinos de supernovas, a transição de neutrinos é determinada apenas pela mistura com o neutrino do elétron, ou seja, os elementos $U_{e i}$.

O sistema é determinado por dois pares de parâmetros:

$$
\begin{array}{r}
\left(\Delta m_{L}^{2}, \sin ^{2} 2 \theta_{L}\right) \cong\left(\Delta m_{\odot}^{2}, \sin ^{2} 2 \theta_{\odot}\right), \\
\left(\Delta m_{H}^{2}, \sin ^{2} 2 \theta_{H}\right) \cong\left(\Delta m_{a t m}^{2}, \sin ^{2} 2 \theta_{13}\right) .
\end{array}
$$

Porém, os dados de oscilação atuais não determinam o espectro de massa e sabor por completo. Por exemplo, há uma ambiguidade no sinal de $\Delta m_{32}^{2}$ (e $\left.\Delta m_{31}^{2}\right)$ pois existem duas possibilidades para a hierarquia de massa do neutrino:

- $\Delta m_{32}^{2}>0$ para $m_{3}>m_{2}, m_{1}$ que corresponde a hierarquia de massa normal,

- $\Delta m_{32}^{2}<0$ para $m_{2}, m_{1}>m_{3}$ que corresponde a hierarquia de massa inversa.

Se a hierarquia é normal, as duas ressonâncias ocorrem no setor de neutrinos. Por outro lado, se a hierarquia é inversa, a ressonância-H ocorre no setor de antineutrinos e a ressonância-L ocorre no setor de neutrinos (Eq. (1.54)).
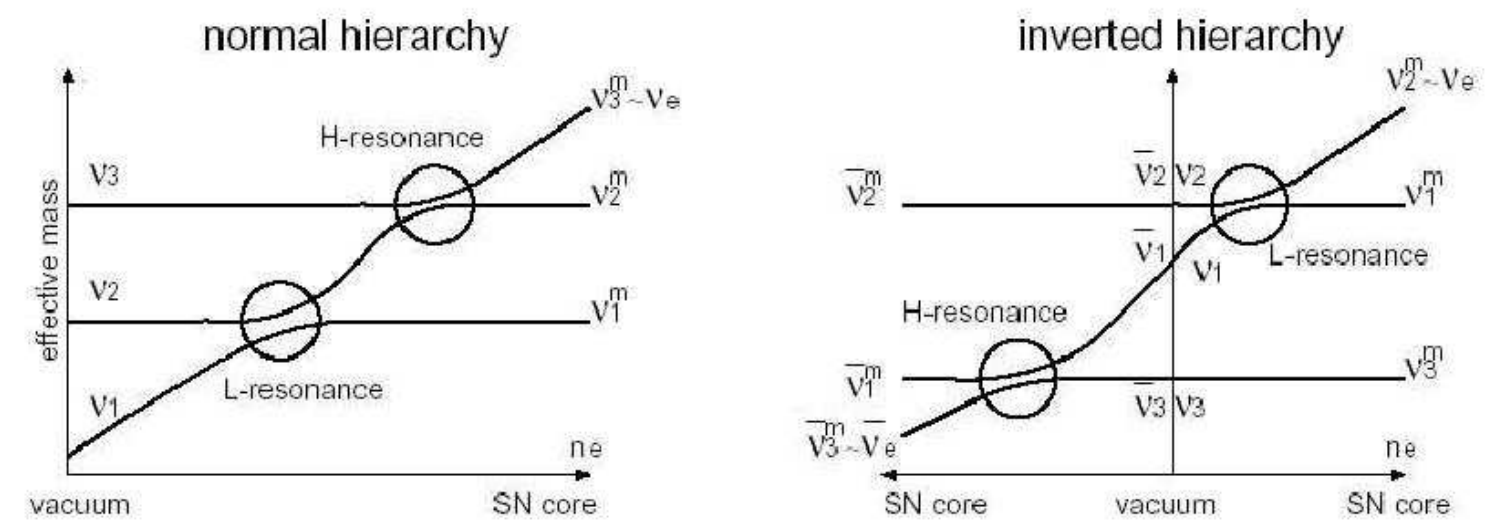

Figura 2.5: Diagrama de ressonâncias para hierarquias normal e inversa. 


\subsubsection{Fatorização da dinâmica}

A natureza hierárquica das diferenças de massa quadradas produzem um desacoplamento natural da dinâmica de conversão em dois canais de ressonância na supernova.

A altas densidades (ressonância $\mathrm{H}$ ), as transições vão afetar apenas os autoestados mais pesados e pode-se reduzir a uma descrição de dois sabores $\left(\nu_{1}, \nu_{3}\right)$. Já nas densidades baixas (ressonância L), apenas o primeiro e segundo autoestados se misturam, reduzindo o problema novamente a dois sabores $\left(\nu_{1}, \nu_{2}\right)$.

Desta forma, a probabilidade de sobrevivência total pode ser fatorizada [63]:

$$
P \approx P_{H} \times P_{L} .
$$

\subsubsection{Limites de adiabaticidade}

As probabilidades de troca fornecidas pela fórmula de Landau - Zener (Eq. (1.60)) podem ser usadas em condições típicas encontradas no manto de supernovas. Contornos de igual probabilidade para diferentes energias de neutrinos podem ser encontrados na Figura (2.6).

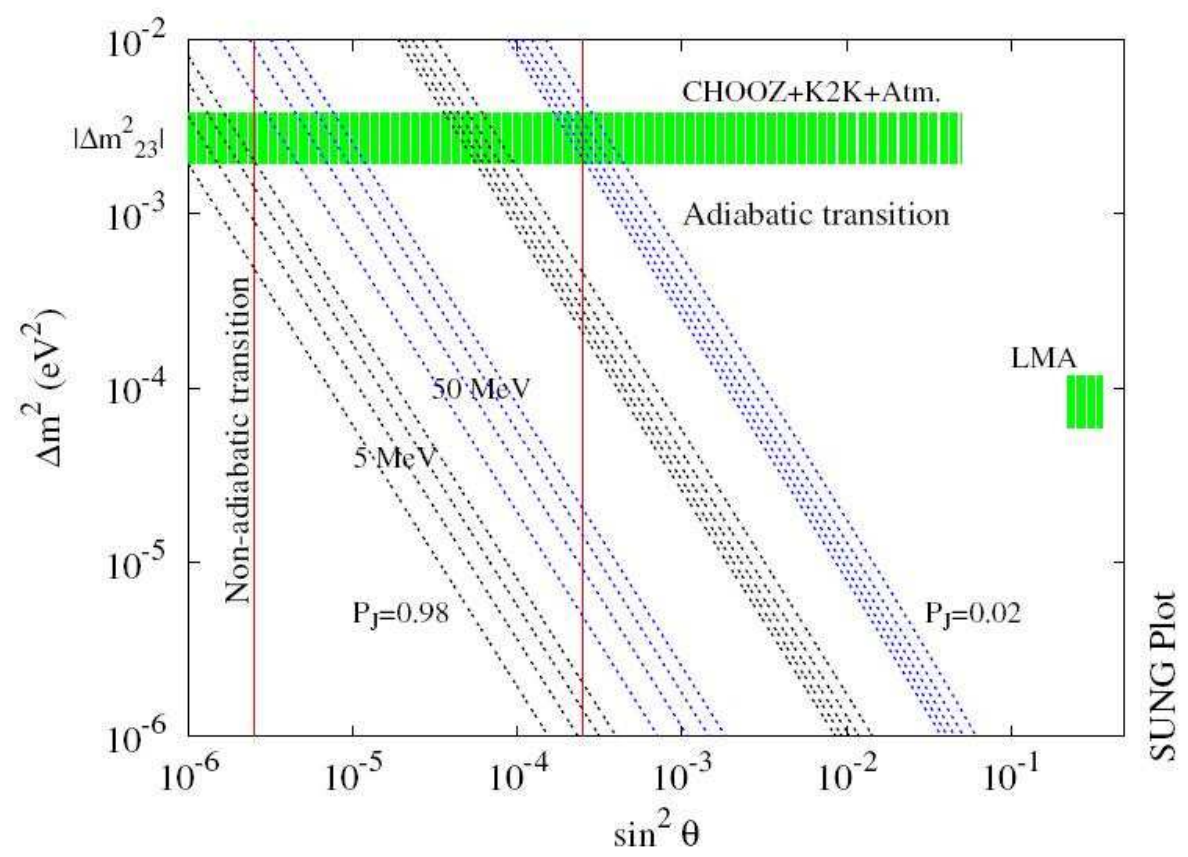

Figura 2.6: Contornos de igual probabilidade de transição $\left(P_{J}\right)$ através de regiões de ressonância com diferentes parâmetros de mistura $\left(\Delta m^{2}, \theta\right)$. Contornos da direita vão de $P_{J}=0,02$ a 0,1 e da esquerda de $P_{J}=0,9$ a 0,98 . Estas probabilidades de transição foram obtidas para um perfil de densidades simples: $\rho(r) Y_{e}=2 \times 10^{4} \mathrm{~g} / \mathrm{cm}^{3}\left(r / 10^{9} \mathrm{~cm}\right)^{3}$ [77]. 
Nesses contornos, vemos que transições governadas pelos parâmetros de mistura solares são completamente adiabáticos, ou seja, $P_{L}=0$. Para ressonâncias envolvendo o ângulo de mistura $\theta_{13}$, a adiabacidade não existe necessariamente. Podemos analizar dois casos extremos:

- $\operatorname{sen}^{2} \theta_{13}>10^{-4}$. Neste caso, adiabaticidade é satisfeita para qualquer energia.

- $\operatorname{sen}^{2} \theta_{13}<10^{-6}$. Agora a adiabaticidade é fortemente violada, implicando que $P_{H} \sim 1$ para quase toda a faixa de energia de neutrinos de supernovas.

- Contornos entre os valores acima apresentam comportamento intermediário, com probabilidade de troca que varia de 0,1 a 0,9 .

Estas conclusões se aplicam também para antineutrinos na hierarquia inversa, sendo que, $\bar{P}_{H}$ será idêntica a $P_{H}$.

No próximo capítulo discutiremos um cálculo analítico aproximado da probabilidade de sobrevivência na condição de máxima violação de adiabaticidade, utilizado na descrição de neutrinos de supernovas.

\subsection{Supernova 1987A}

Em 23 de fevereiro de 1987, um grupo de 11 neutrinos em um intervalo de tempo de 12,4 segundos foi detectado pelo detector Kamiokande II (K2)[90] e outros 8 neutrinos foram observados pelo experimento IMB [91] em um intervalo de 5 segundos. O experimento russo Baksan [92] também detectou 5 eventos, contudo, um forte ruido de fundo no detector pode ter encoberto o sinal. Os eventos detectados encontram-se na Fig. (2.8).

Estes eventos coincidem temporalmente com a supenova 1987A, que foi observada apenas algumas horas depois no ótico, localizada a uma distância de $50 \mathrm{kpc}$ da Terra, na grande Nuvem de Magalhães.

Após resultados de experimentos com neutrinos solares, principalmente dos experimentos SuperKamiokande e SNO, assim como os resultados do experimento com neutrinos de reator, KamLAND, pode-se dizer que os neutrinos da SN 1987A oscilaram até a sua detecção. A transformação de sabor influenciou os sinais observados e, estes efeitos foram levados em consideração nos diversos estudos de análise dos dados feitos $[93,105]$ e na determinação das propriedadess do fluxo original.

Inicialmente foi sugerido que a diferença dos sinais detectados por K2 e IMB poderia ser parcialmente explicada pela oscilação de neutrinos na matéria através da Terra, uma vez que a distância percorrida pelos neutrinos até estes detectores foi diferente (Fig. 2.7). Esta sugestão implicava em um grande ângulo de mistura, que, até então, não era tido como uma idéia favorável. Anos depois, cálculos detalhados [101] mostraram que a LMA é de fato a solução que melhor explica o problema do neutrino solar. Além disso, as características dos neutrinos detectados como a energia, número de eventos e duração da emissão estão de acordo com as predições teóricas da emissão de neutrinos por um colapso estelar. 


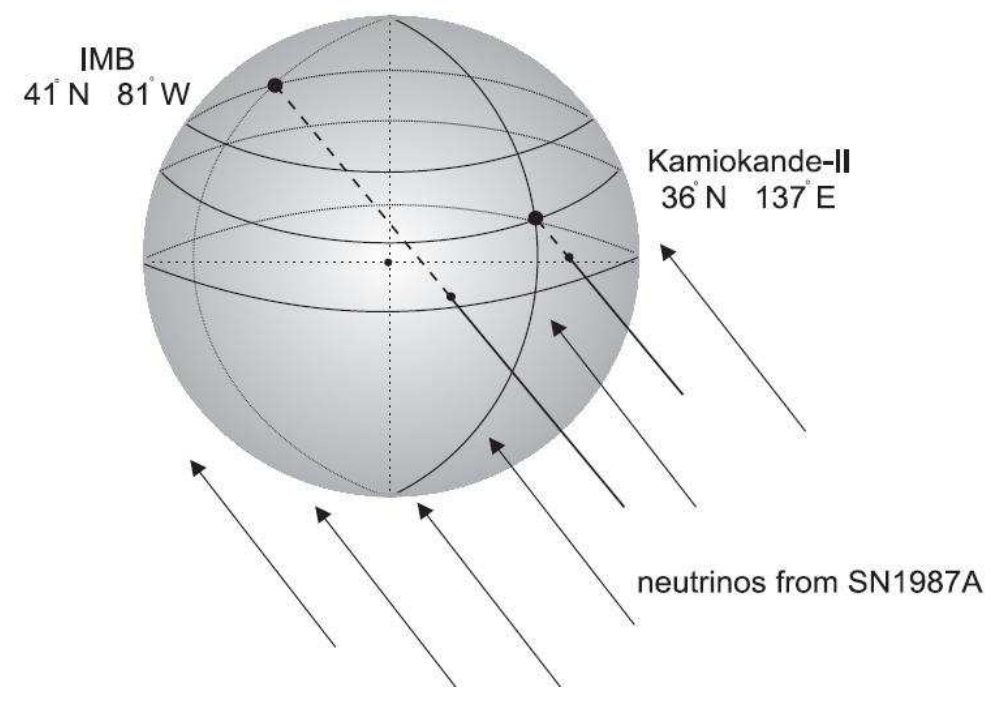

Figura 2.7: Disposição dos detectores Kamiokande II e IMB na Terra no momento do sinal da SN $1987 \mathrm{~A}$.

Outras fontes de deteç̧ão confirmaram os principais aspectos dos modelos de supernova e do cenário atual de processos complexos que ocorrem durante uma explosão. Contudo, a pequena quantidade de neutrinos detectados tem que ser levada em conta com cautela. A pouca estatística, além de fornecer quantidade insatisfatória de características do processo, deixa questões em aberto. A aparente incompatibilidade dos sinais de K2 e IMB será real? A distribuição que se esperava isotrópica apresenta realmente um pico como os dados sugerem? O gap de 7 segundos entre a detecção do oitavo e nono evento no Kamiokande II é real? 


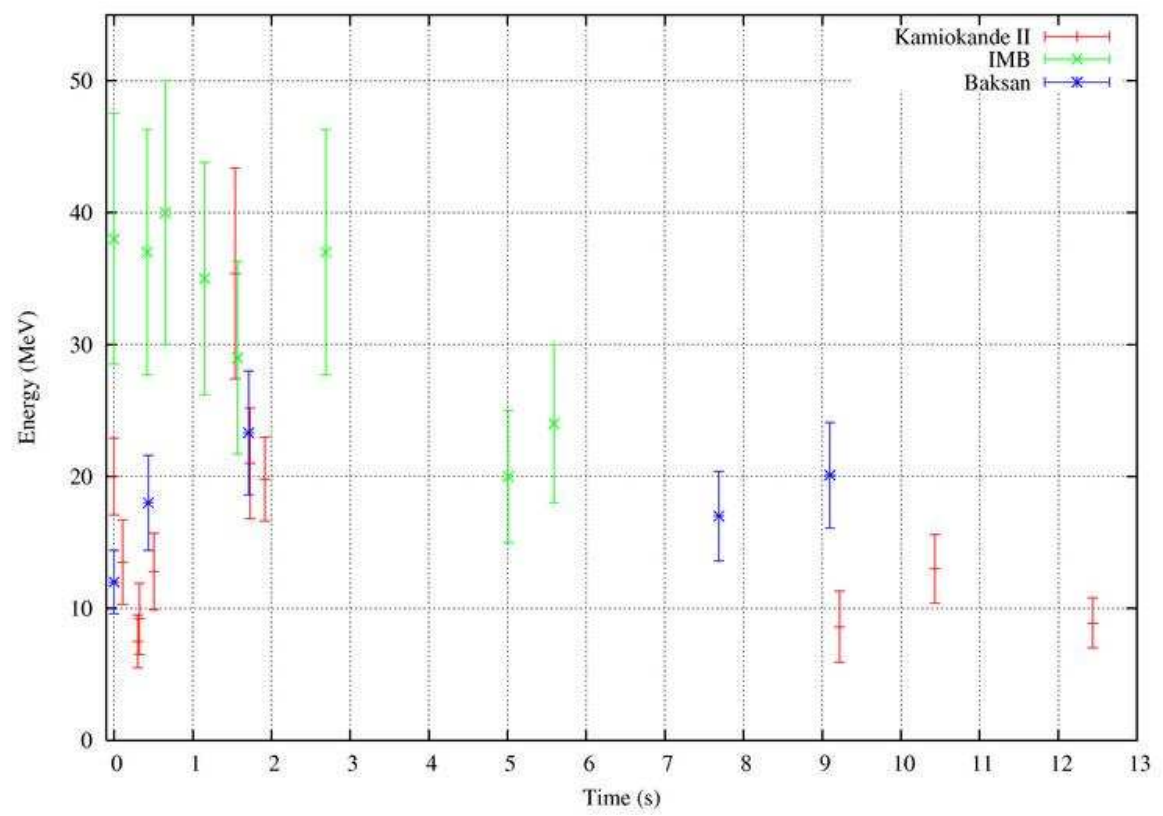

Figura 2.8: Eventos detectados por K2, IMB e Baksan. Nesta montagem, o primeiro neutrino detectado em cada experimento foi condiderado com $t=0$.

Apesar disso é válido tentar realizar a melhor análise possível dos dados da SN 1987A para que possamos tentar determinar o espectro original, não apenas para entender o que aconteceu em 1987, mas para comparar com resultados de futuras detecções. Neutrinos de supernova são eventos raros e cada supernova é única. Sendo a massa da estrela progenitora, luminosidade, rotação, campo magnético, composição química, entre outros aspectos, diferentes, as propriedades do fluxo de neutrinos pode variar, de forma que futuras detecções com maior estatístitica não irão reproduzir as mesmas características da SN 1987A, mas gerarão informação complementar. A comparação de sinais de neutrinos de diferentes supernovas poderá trazer informações importantes para o futuro entendimento dos últimos estágios da evolução estelar, dinâmica do colapso do núcleo e explosão, permitindo avaliar a real importância dos neutrinos em todos estes mecanismos. 


\section{Capítulo 3}

\section{Oscilação de neutrinos em supernovas}

O estudo de oscilação de neutrinos vindos de uma supernova pode se mostrar bastante importante para o entendimento de problemas ainda atuais, como o espectro de massa e mistura dos neutrinos, assim como instrumento para entender a dinâmica da explosão. Isto porque esta fornece situação física única no universo com relação aos efeitos de matéria.

Aproximações para a probabilidade de sobrevivência de neutrinos eletrônicos, $P_{e e}$, tem sido propostas na literatura, em geral, utilizando a aproximação adiabática ou a condição de ressonância [106, 107, 8, 108, 109], com implicações relacionadas apenas com a escala de aplicabilidade. Uma abordagem unificada, não númerica, válida para qualquer parâmetro de oscilação e para um potencial genérico de supernova precisa ser desenvolvida.

Vamos descrever aqui uma abordagem analítica aproximada para o cálculo de $P_{e e}$ baseada em uma probabilidade de transição de dupla exponencial $[110,111]$ e condição de máxima violação de adiabaticidade $[112,113]$.

\subsection{Perfil de densidades}

As oscilações no vácuo podem ser descritas em termos dos caminhos médios percorridos $(x)$ e, sendo $E$ a energia do neutrino, do número de onda de oscilação

$$
k=\frac{\Delta m^{2}}{2 E}
$$

Na matéria, essa dinâmica também dependerá da interação com um potencial efetivo,

$$
V(x)=\sqrt{2} G_{F} N_{e}(x) .
$$

, que como já vimos (Eq. (1.46)) é proporcional a densidade de matéria no meio $\rho(x)$. 
Em geral, a densidade de matéria em uma supernova pode ser aproximadamente descrita por $\rho(x) \approx x^{-3}$ [114]. Trabalharemos aqui com potenciais parametrizados como:

$$
V(x)=V_{0}\left(\frac{x}{R_{\odot}}\right)^{-n}
$$

com $n=3$ e distâncias em unidades do raio solar, $R_{\odot}=6,96 \times 10^{8} \mathrm{~m}$. Vamos usar um exemplo realístico deste potencial [115], vamos supor que a massa ejetada da supernova é $M=14,6 M_{\odot}$ e $V_{0}=1,5 \times 10^{-8} \mathrm{eV}^{2} / \mathrm{MeV}$.

\subsection{Transição entre duas famílias}

Para o caso de transições entre duas famílias $\nu_{e} \rightarrow \nu_{a}$, com autoestados de massa $\nu_{1}$ e $\nu_{2}$, a diferença de massa considerada será $\Delta m^{2}=m_{2}^{2}-m_{1}^{2}>0$.

De acordo com [54], a probabilidade de sobrevivência $P_{e e}$ do estado inicial $\nu_{e}$ na matéria para o estado final $\nu_{e}$ no vácuo pode ser escrita como

$$
P_{e e}(\nu)=(1,0)\left(\begin{array}{cc}
\cos ^{2} \theta & \operatorname{sen}^{2} \theta \\
\operatorname{sen}^{2} \theta & \cos ^{2} \theta
\end{array}\right)\left(\begin{array}{cc}
1-P_{c}(\nu) & P_{c}(\nu) \\
P_{c}(\nu) & 1-P_{c}(\nu)
\end{array}\right)\left(\begin{array}{cc}
\cos ^{2} \theta_{m} & \operatorname{sen}^{2} \theta_{m} \\
\operatorname{sen}^{2} \theta_{m} & \cos ^{2} \theta_{m}
\end{array}\right)\left(\begin{array}{l}
1 \\
0
\end{array}\right),
$$

sendo $P_{c}(\nu)$ a probabilidade de troca ou de salto entre 2 autoestados de massa para neutrinos ${ }^{1}$ $\left[P_{c}(\nu)=P\left(\nu_{2 m} \rightarrow \nu_{1 m}\right)\right]$ e $\theta_{m}$ o ângulo de mistura na matéria, definido como

$$
\begin{aligned}
\operatorname{sen} 2 \theta_{m} & =\frac{\operatorname{sen} 2 \theta}{\sqrt{(\cos 2 \theta-V / k)^{2}+\operatorname{sen}^{2} 2 \theta}}, \\
\cos 2 \theta_{m} & =\frac{\cos 2 \theta-V / k}{\sqrt{(\cos 2 \theta-V / k)^{2}+\operatorname{sen}^{2} 2 \theta}} .
\end{aligned}
$$

A alta densidade do núcleo da supernova implica que $\frac{V}{k}>>1$, de forma que $\operatorname{sen} 2 \theta_{m} \simeq 0$ e $\cos 2 \theta_{m} \simeq-1$. Desta forma, a probabilidade de sobrevivência será:

$$
P_{e e}(\nu)=\cos ^{2} \theta P_{c}(\nu)+\operatorname{sen}^{2} \theta\left(1-P_{c}(\nu)\right) .
$$

Existem resultados para o cálculo de $P_{c}(\nu)$ baseados na integração numérica das equações de evolução do neutrino através do perfil do potencial da supernova $[116,117,118,119]$. Contudo, estes cálculos, que são exatos em princípio, são computacionalmente trabalhosos e não representam uma aproximação eficiente para o caso de supernovas.

\footnotetext{
${ }^{1}$ Os casos $P_{c} \sim 0$ e $P_{c} \neq 0$ correspondem aos casos adiabático e não adiabático respectivamente.
} 
Optamos então por uma aproximação analítica dos resultados numéricos para $P_{c}(\nu)$, aplicável em todo o espaço de parâmetros. Tal aproximação deriva de um aperfeiçoamento na compreensão de transições não adiabáticas.

Nossa base analítica partirá da identificação de um ponto $x_{p}$ onde o potencial se iguala ao número de onda k, pois neste ponto haverá a potencialização dos efeitos de matéria [57, 58]

$$
V\left(x_{p}\right)=k \text {. }
$$

Para este ponto foi calculada a caracterização realística da mudança da densidade de elétrons ao longo da trajetória de neutrinos, chamada fator de escala de densidade

$$
r=\left|\frac{1}{V(x)} \frac{d V(x)}{d x}\right|_{x=x_{p}}^{-1},
$$

que foi inserido na parametrização de dupla-exponencial para $P_{c}(\nu)$ :

$$
P_{c}(\nu)=\frac{\exp \left(2 \pi r k \cos ^{2} \theta\right)-1}{\exp (2 \pi r k)-1}
$$

Os resultados encontram-se no gráfico 3.1. Esta parametrização foi inicialmente derivada no contexto de neutrinos solares [110,111], e tem sido aplicada a transições de neutrinos de altas energias provenientes do decaimento de partículas massivas hipotéticas presas no Sol [120] e também a neutrinos de supernovas [121, 122, 123]. Este, por sua vez, além de ser um bom Ansatz para perfis de densidade genéricos, é válido para qualquer ângulo de mistura, reproduz o limite de extrema não adiabaticidade para pequenos $k$ 's e também reproduz a função de uma exponencial do limite Landau-Zener para pequenos $\theta$ 's.

Uma vez calculado $P_{c}(\nu)$, obtemos então $P_{e e}$ através da Eq. (3.7). Esta descrição analítica é aplicável para qualquer potencial $V(x)$ na forma dada pela Eq. (3.3). 

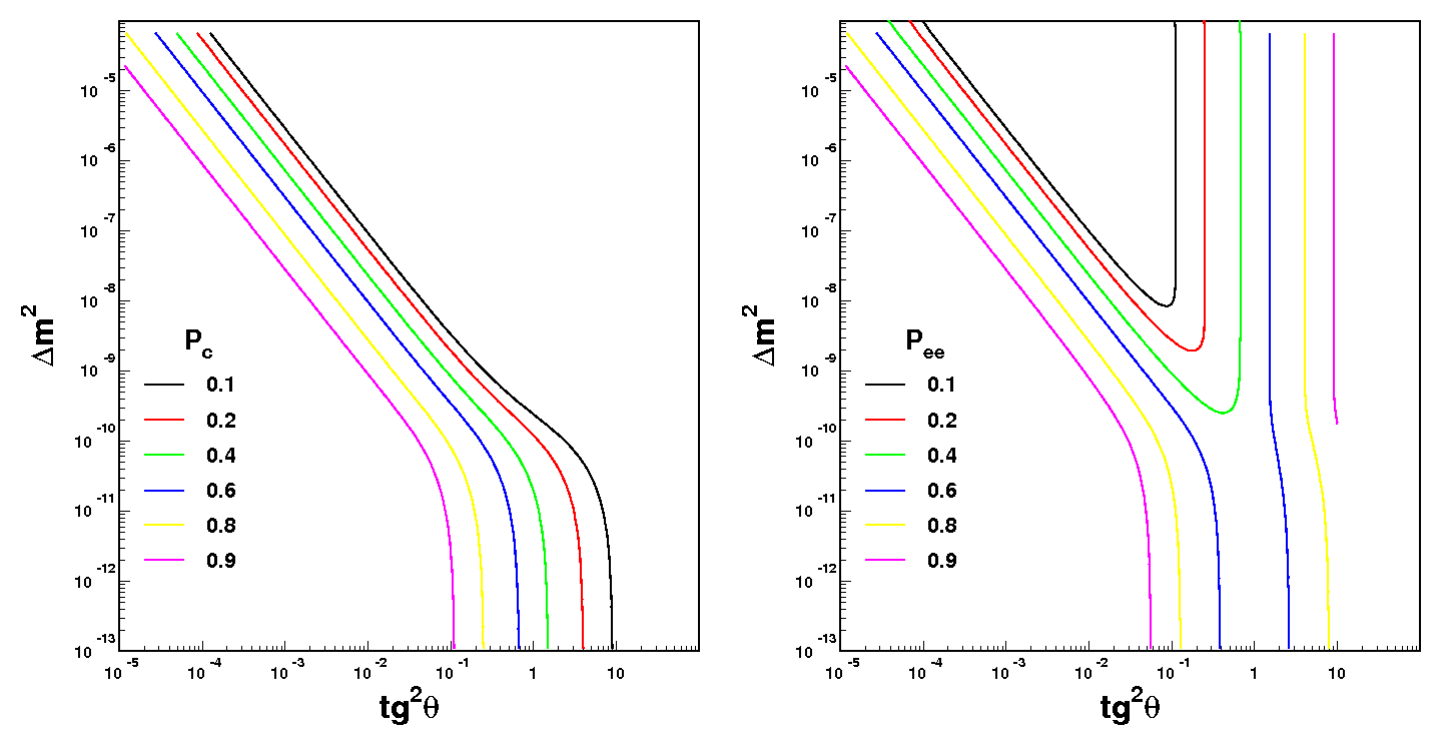

Figura 3.1: Curvas de nível mostram a dependência da probabilidade de transição do neutrino, $P_{c}$, e da probabilidade de sobrevivência, $P_{e e}$, com os parâmetros de mistura $\left(\Delta m^{2}, \tan ^{2} \theta\right)$ para transições entre dois sabores e energia do neutrino $E=15 \mathrm{MeV}$.

A extensão do modelo analítico para antineutrinos pode ser obtido através da mudança $V / k \rightarrow$ $-V / k$ em $P_{e e}$. Por convenção, mantendo $V>0$, isso implica que

$$
P_{\bar{e} \bar{e}}\left(+\Delta m^{2}\right) \equiv P_{e e}\left(-\Delta m^{2}\right) \equiv P_{e e}\left(+\Delta m^{2}\right)_{1 \leftrightarrow 2},
$$

que corresponde a $U_{e 1}^{2} \leftrightarrow U_{e 2}^{2}$ e $\operatorname{sen}^{2} \theta \leftrightarrow \cos ^{2} \theta$.

Teremos então que

$$
P_{e e}(\bar{\nu}) \equiv P_{e e}(\nu)_{1 \leftrightarrow 2}=\operatorname{sen}^{2} \theta P_{c}(\bar{\nu})+\cos ^{2} \theta\left(1-P_{c}(\bar{\nu})\right),
$$

e

$$
P_{c}(\bar{\nu}) \equiv P_{c}(\nu)_{1 \leftrightarrow 2}=\frac{\exp \left(2 \pi r k \operatorname{sen}^{2} \theta\right)-1}{\exp (2 \pi r k)-1} .
$$

As isolinhas de $P_{c}(\bar{\nu})$ são imagens espelhadas em torno do eixo $\tan ^{2} \theta=1$ das obtidas para $P_{c}(\nu)$ na Figura (3.1). 


\subsection{Transição entre três famílias}

A generalização dos resultados de transições entre dois sabores para três é importante pois os neutrinos de supernova podem fornecer testes peculiares de oscilação em três sabores, devido a ampla dinâmica de $V(x)$ nas estrelas em colapso.

Assumimos mistura de três neutrinos ativos $\left(\nu_{e}, \nu_{\mu}, \nu_{\tau}\right)$ e três autoestados de massa $\left(\nu_{1}, \nu_{2}\right.$, $\nu_{3}$ ) através da matriz de mistura U. Os elementos de matriz $U_{e i}$ são parametrizados em termos dos ângulos de mistura $\theta_{12}$ e $\theta_{13}$,

$$
\begin{array}{r}
U_{e 1}^{2}=\cos ^{2} \theta_{13} \cos ^{2} \theta_{12}, \\
U_{e 2}^{2}=\cos ^{2} \theta_{13} \operatorname{sen}^{2} \theta_{12}, \\
U_{e 3}^{2}=\operatorname{sen}^{2} \theta_{13} .
\end{array}
$$

Os parâmetros ficam completos incluindo as duas diferenças de massa $\Delta m_{21}^{2}$ e $\Delta m_{31}^{2} \simeq \Delta m_{32}^{2}$ e fixando um potencial $V(x)$. Os parâmetros espaciais $S_{3 \nu}$ para descrever uma supernova são:

$$
S_{3 \nu}=\left(\Delta m_{21}^{2}, \Delta m_{32}^{2}, \theta_{12}, \theta_{13}, V\right) \text {. }
$$

Como consequência da separação hierárquica assumida nas Eqs. (2.13)-(2.16), a dinâmica de três sabores pode ser reduzida a duas dinâmicas de dois sabores, low $(\mathrm{L})$ e high $(\mathrm{H})$. A fatorização dos parâmetros espaciais fica assim:

$$
S_{3 \nu} \cong L_{2 \nu} \otimes H_{2 \nu}=\left(\Delta m_{21}^{2}, \theta_{12}, V \cos ^{2} \theta_{13}\right) \otimes\left(\Delta m_{32}^{2}, \theta_{13}, V\right)
$$

Teremos então os números de onda definidos por:

$$
k_{L}=\frac{\Delta m_{21}^{2}}{2 E}
$$

e

$$
k_{H}=\frac{\Delta m_{32}^{2}}{2 E} .
$$

\subsubsection{Transições com hierarquia normal para neutrinos}

Para a hierarquia normal, utilizando a fatorização da Eq. (3.18), a probabilidade de sobrevivência será [54] 
$P_{e e}(\nu)=\left(U_{e 1}^{2}, U_{e 2}^{2}, U_{e 3}^{2}\right)\left(\begin{array}{ccc}1-P_{L}(\nu) & P_{L}(\nu) & 0 \\ P_{L}(\nu) & 1-P_{L}(\nu) & 0 \\ 0 & 0 & 1\end{array}\right)\left(\begin{array}{ccc}1 & 0 & 0 \\ 0 & 1-P_{H}(\nu) & P_{H}(\nu) \\ 0 & P_{H}(\nu) & 1-P_{H}(\nu)\end{array}\right)\left(\begin{array}{c}U_{e 1, m}^{2} \\ U_{e 2, m}^{2} \\ U_{e 3, m}^{2}\end{array}\right)$,

sendo $P_{L}$ e $P_{H}$ as probabilidades de troca para transições low e high respectivamente, e os elementos $U_{e i, m}^{2}$ como nas Eqs. (3.14)-(3.17) mas para ângulos de mistura na matéria, que são dados por

$$
\begin{aligned}
\operatorname{sen} 2 \theta_{12, m} & =\frac{\operatorname{sen} 2 \theta_{12}}{\sqrt{\left(\cos 2 \theta_{12}-\cos ^{2} \theta_{13} V / k_{L}\right)^{2}+\operatorname{sen}^{2} 2 \theta_{12}}}, \\
\cos 2 \theta_{12, m} & =\frac{\cos 2 \theta_{12}-V / k_{L}}{\sqrt{\left(\cos 2 \theta_{12}-\cos ^{2} \theta_{13} V / k_{L}\right)^{2}+\operatorname{sen}^{2} 2 \theta_{12}}}
\end{aligned}
$$

e

$$
\begin{aligned}
\operatorname{sen} 2 \theta_{13, m} & =\frac{\operatorname{sen} 2 \theta_{13}}{\sqrt{\left(\cos 2 \theta_{13}-V / k_{H}\right)^{2}+\operatorname{sen}^{2} 2 \theta_{13}}}, \\
\cos 2 \theta_{13, m} & =\frac{\cos 2 \theta_{13}-V / k_{H}}{\sqrt{\left(\cos 2 \theta_{13}-V / k_{H}\right)^{2}+\operatorname{sen}^{2} 2 \theta_{13}}} .
\end{aligned}
$$

A condição inicial, considerando altíssimas densidades, $V / k_{L, H}>>1$ leva a $\cos 2 \theta_{13, m} \simeq-1 \simeq$ $\cos 2 \theta_{12, m}$ e para a expressão

$$
P_{e e}(\nu)=U_{e 1}^{2} P_{L}(\nu) P_{H}(\nu)+U_{e 2}^{2}\left[1-P_{L}(\nu)\right] P_{H}(\nu)+U_{e 3}^{2}\left[1-P_{H}(\nu)\right] .
$$

$P_{L}(\nu)$ e $P_{H}(\nu)$ podem ser expressos analiticamente como

$$
P_{L}(\nu)=\frac{\exp \left(2 \pi r_{L} k_{L} \cos ^{2} \theta_{12}\right)-1}{\exp \left(2 \pi r_{L} k_{L}\right)-1}
$$

e

$$
P_{H}(\nu)=\frac{\exp \left(2 \pi r_{H} k_{H} \cos ^{2} \theta_{13}\right)-1}{\exp \left(2 \pi r_{H} k_{H}\right)-1}
$$

sendo

$$
r_{L, H}=-\left[\frac{1}{V(x)} \frac{d V(x)}{d x}\right]_{x=x_{L}, x_{H}}^{-1},
$$

com os pontos $x_{L}$ e $x_{H}$ definidos a partir da condição

$$
V\left(x_{L, H}\right)=k_{L, H}
$$




\subsubsection{Transições com hierarquia normal para antineutrinos}

O caso para antineutrinos é obtido de forma análoga, apenas substituindo $V / k_{L, H} \rightarrow-V / k_{L, H}$. Os ângulos de mistura, neste caso, serão:

$$
\begin{aligned}
\operatorname{sen} 2 \bar{\theta}_{12, m} & =\frac{\operatorname{sen} 2 \theta_{12}}{\sqrt{\left(\cos 2 \theta_{12}+\cos ^{2} \theta_{13} V / k_{L}\right)^{2}+\operatorname{sen}^{2} 2 \theta_{12}}}, \\
\cos 2 \bar{\theta}_{12, m} & =\frac{\cos 2 \theta_{12}+V / k_{L}}{\sqrt{\left(\cos 2 \theta_{12}+\cos ^{2} \theta_{13} V / k_{L}\right)^{2}+\operatorname{sen}^{2} 2 \theta_{12}}}
\end{aligned}
$$

e analogamente para $\bar{\theta}_{13, m}$. A condição inicial de altíssimas densidades leva : $\cos 2 \bar{\theta}_{13, m} \simeq+1 \simeq$ $\cos 2 \bar{\theta}_{12, m}$, e portanto:

$$
P_{\bar{e} \bar{e}}=U_{e 1}^{2}\left[1-P_{L}(\bar{\nu})\right]+U_{e 2}^{2} P_{L}(\bar{\nu}),
$$

A forma analítica de $P_{L}(\bar{\nu})$ é obtida, após as mudanças necessárias, da Eq. (3.13),

$$
P_{L}(\bar{\nu})=\frac{\exp \left(2 \pi r_{L} k_{L} \operatorname{sen}^{2} \theta_{12}\right)-1}{\exp \left(2 \pi r_{L} k_{L}\right)-1}
$$

\subsubsection{Transições com hierarquia inversa para neutrinos}

Como vimos, para um espectro de massa e ângulos de mistura fixos, as probabilidades de neutrinos e antineutrinos são convertidas umas nas outras trocando o sinal de $V / k_{L, H}$, o que equivale a trocar o sinal da diferença de massa. Da mesma forma, a troca do sinal da diferença de massa $\Delta m_{32}^{2}$ equivale a uma mudança de hierarquia. Devido as propriedades de simetria, teremos:

$$
P_{e e}\left(+\Delta m_{21}^{2},-\Delta m_{32}^{2}\right) \equiv P_{\bar{e} \bar{e}}\left(-\Delta m_{21}^{2},+\Delta m_{32}^{2}\right) \equiv P_{\bar{e} \bar{e}}\left(+\Delta m_{21}^{2},+\Delta m_{32}^{2}\right)_{1 \leftrightarrow 2} .
$$

Vemos então que a probabilidade de sobrevivência para o neutrino do elétron na hierarquia inversa é igual a probabilidade de sobrevivência para o antineutrino do elétron na hierarquia normal com a inversão $U_{e 1}^{2} \leftrightarrow U_{e 2}^{2}$.

$$
P_{e e}=U_{e 2}^{2}\left[1-P_{L}(\nu)\right]+U_{e 1}^{2} P_{L}(\nu),
$$

sendo $P_{L}(\nu)$ definido na Eq. (3.27). 


\subsubsection{Transições com hierarquia inversa para antineutrinos}

Analogamente ao caso de neutrinos, é fácil ver que

$$
P_{\bar{e} \bar{e}}\left(+\Delta m_{21}^{2},-\Delta m_{32}^{2}\right) \equiv P_{e e}\left(-\Delta m_{21}^{2},+\Delta m_{32}^{2}\right) \equiv P_{e e}\left(+\Delta m_{21}^{2},+\Delta m_{32}^{2}\right)_{1 \leftrightarrow 2},
$$

que aplicada à equação (3.26) fica

$$
P_{\bar{e} \bar{e}}=U_{e 2}^{2} P_{L}(\bar{\nu}) P_{H}(\nu)+U_{e 1}^{2}\left[1-P_{L}(\bar{\nu})\right] P_{H}(\nu)+U_{e 3}^{2}\left[1-P_{H}(\nu)\right]
$$

com $P_{L}(\bar{\nu})$ e $P_{H}(\nu)$ definidos nas Eqs. (3.34) e (3.28), respectivamente.

É importante notar que a mudança $1 \leftrightarrow 2$ não faz diferença em $P_{H}$, que é definido em termos de $m_{3}^{2}-m_{1,2}^{2}$ e de $U_{e 3}^{2}$, de forma que

$$
P_{H}(\bar{\nu})=P_{H}(\nu) \equiv P_{H}
$$

\subsection{Transformação de sabor coletiva em neutrinos de supernova}

Além de oscilações que geram troca de sabor por efeito MSW, transições de sabor dentro de uma SN podem ocorrer por transformação de sabor coletiva.

A probabilidade de troca de sabor não depende mais apenas do perfil de densidade do meio, mas também da intensidade do fluxo de neutrinos. Interações neutrino-neutrino $(\nu-\nu)$ geram termos não lineares nas equações de movimento $[124,125]$ que geram transformações de sabor coletivas $[126,127,128,129,130,131,132,133,134,135,136,137,138]$. Este tipo de interação ocorre em densidades muito grandes de neutrinos, como por exemplo no universo primordial, objetos muito compactos e após o colapso gravitacional, em particular, o núcleo de prótons-nêutrons resultante de uma SN [139, 132, 131].

Em um caso típico de fluxos transformados numericamente, o fluxo primário de $\nu_{e}$ abaixo de uma energia divisória (split energy $E_{\text {split }}$ ) emerge da região de alta densidade de neutrinos em seu sabor original, enquanto que acima desta energia $E_{\text {split }}$, ele é completamente transformado em $\nu_{x}$. A energia $E_{\text {split }}$ é fixada pela chamada conservação do número leptônico de sabor. Para um ângulo de mistura suficientemente pequeno as transformações de sabor coletivas têm a propriedade de conservar o número de sabor, pois apenas pares $\nu_{e} \bar{\nu}_{e}$ são transformados em pares $\nu_{x} \bar{\nu}_{x}$, fazendo com que o excesso do fluxo seja conservado [128]. 


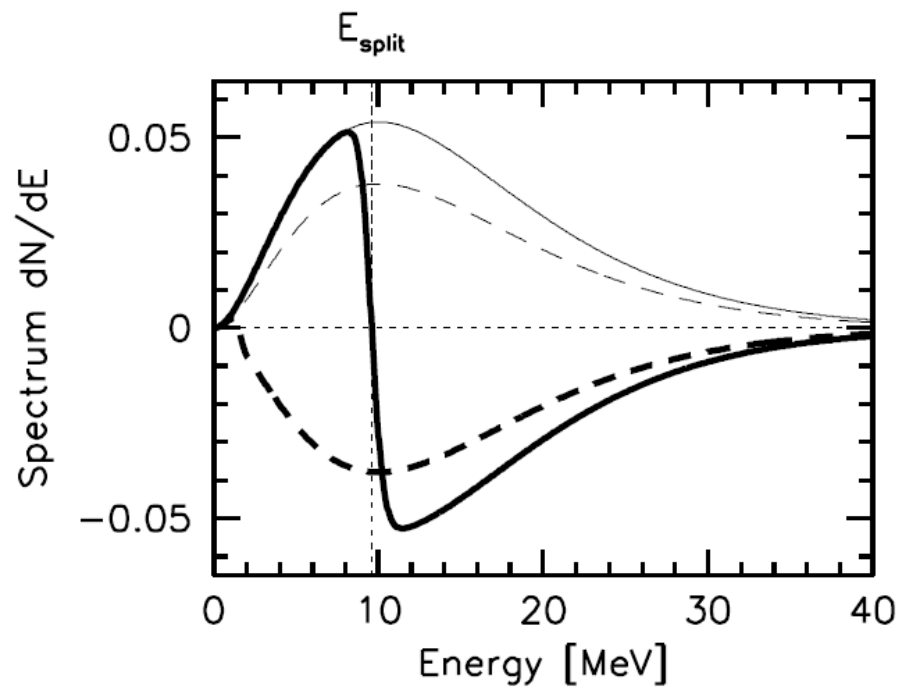

Figura 3.2: Espectro de neutrinos na neutrinosfera (linhas finas) e após atravessarem a região densa (linhas grossas). As linhas sólidas representam neutrinos e as pontilhadas os antineutrinos. Espectro positivo corresponde a (anti)neutrinos eletrônicos e espectro negativo corresponde a (anti)neutrinos $x[128]$.

Este fenômeno fica mais claro quando o espectro é representado em termos do número de onda de oscilação $k=\Delta m^{2} / 2 E$ [131]. Nas equações de evolução a única diferença entre neutrinos e antineutrinos aparece no sinal negativo de $k$, levando a pensar em um espectro contínuo $-\infty<k<$ $+\infty$.

O fenômeno de divisão espectral não chega a ser um efeito puramente novo, mas uma nova forma de olhar para o usual efeito MSW expressando sua dependência energética em termos do número de onda de oscilação $k$ e identificando antineutrinos com frequências negativas.

Estes efeitos, embora interessantes, não foram considerados em nosso trabalho. 


\section{Capítulo 4}

\section{Estudo de oscilação de neutrinos para supernovas galáticas com um potencial estático}

Uma vez que ainda existem muitas questões em aberto sobre a dinâmica da supernova, uma futura explosão pode trazer novas informações principalmente referentes a parâmetros que refletem a dinâmica da explosão assim como parâmetros de oscilação de neutrinos. Fizemos aqui cálculos para avaliar o potencial de uma possível detecção de neutrinos provenientes de uma supernova galática por um detector de água baseado no efeito Cherenkov. O processo de detecção é o decaimento beta inverso. Trabalharemos nos casos limites de total adiabaticidade e não adiabaticidade, cada um em hierarquia normal e inversa.

O propósito deste capítulo é investigar com algum detalhe a dependência energética do espectro de neutrinos para uma supernova de potencial estático. Queremos também avaliar a possibilidade de se obter limites para os parâmetros de supernova e oscilação de neutrinos a partir da detecção de antineutrinos eletrônicos de uma futura explosão de supernova.

\subsection{Definições iniciais}

Nosso objetivo aqui é calcular o número esperado de neutrinos, emitidos durante a fase de resfriamento de uma supernova tipo II, que poderiam ser observados por um detector Cherenkov com 540 kton de água, correspondente ao esperado para o Hyper-Kamiokande [140]. Vamos estudar apenas a observação de antineutrinos eletrônicos através da reação de decaimento beta inverso

$$
\bar{\nu}_{e}+p \rightarrow n+e^{+}
$$

A luminosidade total $E_{b}$ esperada nesta fase é estimada entre $(1-5) \times 10^{53} \mathrm{ergs}$ [8]. Assumindo $x=\nu_{\mu}, \nu_{\tau}, \overline{\nu_{\mu}}, \overline{\nu_{\tau}}$ indistinguíveis, temos $E_{b}=L_{e}+L_{\bar{e}}+4 L_{x}[141,142]$. Sendo $L_{e}, L_{\bar{e}}$ e $L_{x}$ as luminosidades da supernova para cada espécie de neutrino. 
Para o caso mais simples, a luminosidade será [143]

$$
L(t)=\frac{E_{b}}{6 \tau} \exp (-t / \tau)
$$

sendo $\tau=3$ s o tempo de decaimento [149].

Para o espectro de energia de neutrinos emitidos pela SN, usamos a distribuição de Fermi-Dirac distorcida ("pinched") [8]

$$
\phi_{i}^{0}(E)=\frac{1}{F_{3}\left(\eta_{i}\right) T_{i}^{4}} \frac{E^{2}}{\exp \left(E / T_{i}-\eta_{i}\right)}, i=e, \bar{e}, x
$$

sendo $F_{3}$ uma constante de normalização de valor final $F_{3}=5,68$ [142], $T_{i}$ a temperatura para a espécie $i$, que está relacionada com a energia média $<E_{i}>$ por uma constante $\left(T_{i}=\frac{<E_{i}>}{3,15}\right)$ [142], e $\eta_{i}$ o parâmetro de distorção, que vamos considerar nulo em nosso estudo.

Em geral, a emissão da SN no óptico é absorvida pela poeira e ofuscada pela luz do centro da galáxia de forma que a distância D desta à Terra será, com grande probabilidade, incerta. Por isso, usou-se o parâmetro $E_{b} / D^{2}$ que pode ser obtido após a deteç̧ão. Desta forma, o fluxo inicial, sem oscilação, será:

$$
F_{\nu_{i}}^{0}=\frac{L(t)}{4 \pi D^{2}} \phi_{i}^{0}(E)=\frac{L(t)}{4 \pi} \frac{E_{b} / D^{2}}{E_{b}} \phi_{i}^{0}(E)
$$

Como vimos, para uma supernova, apenas os parâmetros $\Delta m_{31}^{2}$ e $\theta_{13}$ são relevantes para a ressonância [77]. Neste caso, a ressonância H vai ocorrer para neutrinos (antineutrinos) na hierarquia normal (invertida), e haverá uma probabilidade $P_{H}$ de transição entre os autoestados de massa. Já a probabilidade $P_{L}$ que está relacionada com os parâmetros $\Delta m_{21}^{2}$ e $\theta_{12}$ será sempre $P_{L} \sim 0$, ou seja, estaremos sempre no caso adiabático. A probabilidade de transição foi parametrizada como [140]

$$
P_{H}=\exp \left[-\operatorname{sen}^{2} \theta_{13}\left(\frac{1,08 \times 10^{7}}{E}\right)^{2 / 3}\left(\frac{\left|\Delta m_{31}^{2}\right|}{10^{-3}}\right)^{2 / 3}\right]
$$

com $E$ em $\mathrm{MeV}$ e $\Delta m_{31}^{2} \mathrm{em} \mathrm{eV}^{2}$. Esta relação foi obtida para o caso de um potencial estático do tipo $x^{-n}$ com $n=3$ (Eq. (3.4)).

Mostramos as probabilidades de sobrevivência $P_{e e}$ e $P_{\bar{e} \bar{e}}$ de $\nu_{e}$ e $\overline{\nu_{e}}$ para a hierarquia normal e inversa nos gráficos das Figuras (4.1) e (4.2). Para Hierarquia Normal (HN)

$$
\begin{array}{r}
P_{\bar{e} \bar{e}}=\cos ^{2} \theta_{12} \cos ^{2} \theta_{13} \\
P_{e e}=P_{H} \operatorname{sen}^{2} \theta_{12} \cos ^{2} \theta_{13}+\left(1-P_{H}\right) \operatorname{sen}^{2} \theta_{13},
\end{array}
$$

e para Hierarquia Inversa(HI) 


$$
\begin{array}{r}
P_{\bar{e} \bar{e}}=P_{H} \cos ^{2} \theta_{12} \cos ^{2} \theta_{13}+\left(1-P_{H}\right) \operatorname{sen}^{2} \theta_{13} \\
P_{e e}=\operatorname{sen}^{2} \theta_{12} \cos ^{2} \theta_{13} .
\end{array}
$$

Para o caso em que estamos interessados, detecção de $\overline{\nu_{e}}$, o fluxo que chega a Terra será, não considerando efeitos de matéria na Terra,

$$
F_{\overline{\nu_{e}}}=F_{\overline{\nu_{e}}}^{0} P_{\bar{e} \bar{e}}+F_{\overline{\nu_{x}}}^{0}\left(1-P_{\bar{e} \bar{e}}\right)
$$

para ambas hierarquias.

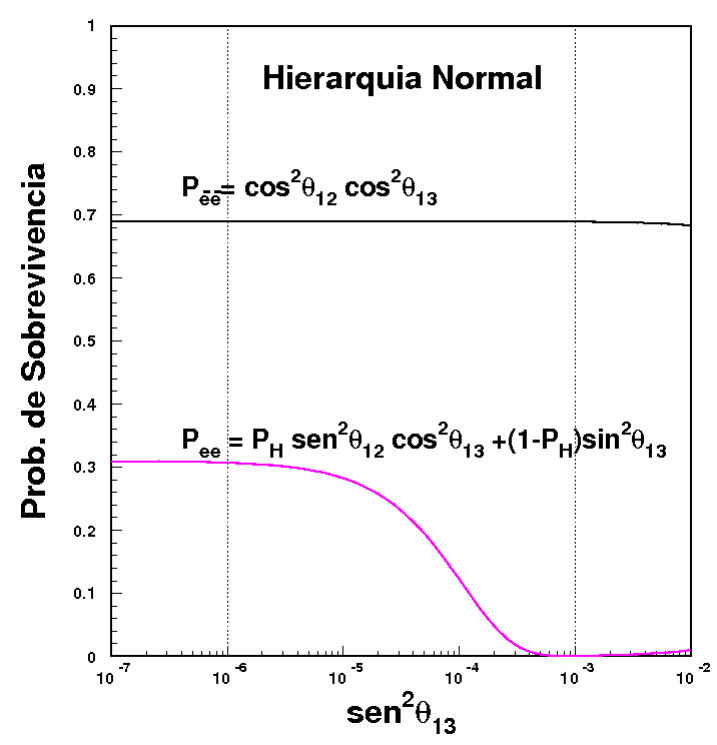

Figura 4.1: Probabilidade de sobrevivência de neutrinos $\left(P_{e e}\right)$ e antineutrinos $\left(P_{\overline{e e}}\right)$ do elétron para hierarquia normal. O cálculo foi feito para energia $E=60 \mathrm{MeV}$. Consideramos aqui $\operatorname{sen}^{2} \theta_{12}=0,31$, $\Delta m_{21}^{2}=8,0 \times 10^{-5} \mathrm{eV}^{2}$ e $\left|\Delta m_{31}^{2}\right|=3,0 \times 10^{-3} \mathrm{eV}^{2}[140]$. 


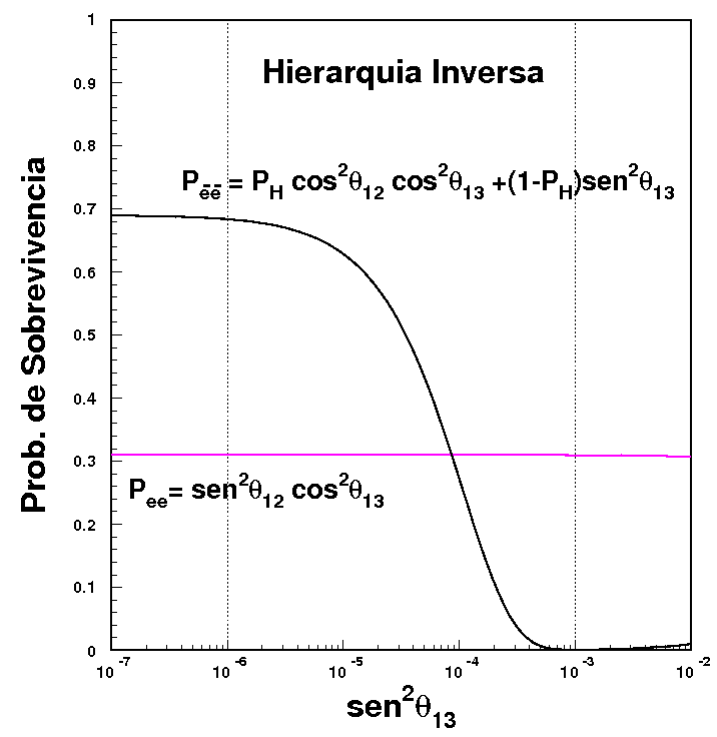

Figura 4.2: Análogo à Fig. (4.1) mas para hierarquia inversa.

\subsection{Número de eventos total e espectro energético esperado}

Discutiremos aqui o método e os parâmetros utilizados para o estudo de neutrinos de uma supernova próxima, na nossa galáxia.

O fluxo de neutrinos de uma SN (Eq. (4.10)) pode ser parametrizado por quatro variáveis que descrevem a $\mathrm{SN},\left\langle E_{e}\right\rangle,\left\langle E_{\bar{e}}\right\rangle,\left\langle E_{x}\right\rangle$ e $E_{b} / D^{2}$, além de dois parâmetros relativos à oscilação, $\operatorname{sen}^{2} \theta_{13},\left|\Delta m_{31}^{2}\right|$, e a hierarquia.

A seção de choque para a reação $\overline{\nu_{e}}+p \rightarrow e^{+}+n$ [144] foi calculada até primeira ordem de teoria de perturbação. Até primeira ordem de $1 / M, \operatorname{com} M$ a massa do nucleon, a energia e momento do pósitron é

$$
\begin{array}{r}
E_{e}^{(0)}=E_{\nu}-\Delta, \Delta=M_{n}-M_{p} ; \\
E_{e}^{(1)}=E_{e}^{(0)}\left[1-\frac{E_{\nu}}{M}\left(1-v_{e}^{(0)} \cos \theta\right)\right]-\frac{\left(\Delta^{2}-M_{e}^{2}\right)}{2 M} ; \\
p_{e}^{(i)}=\sqrt{E_{e}^{(i) 2}-M_{e}^{2}} ; \\
v_{e}^{(i)}=\frac{p_{e}^{(i)}}{E_{e}^{(i)}}, \quad i=0,1 ;
\end{array}
$$

sendo $E_{\nu}$ a energia do antineutrino, $M_{n}, M_{p}$ e $M_{e}$ as massas do nêutron, próton e elétron respectivamente. 
Desta forma, podemos representar a seção de choque final em ordem zero e em primeira ordem, negligenciando os termos quadráticos como [144]

$$
\begin{array}{r}
\sigma_{T O T}^{(0)}=\sigma_{0}\left(f^{2}+3 g^{2}\right) E_{e}^{(0)} p_{e}^{(0)} \\
\sigma_{T O T}^{(1)}=\frac{\sigma_{0}}{2} E_{e}^{(0) 2}\left[2\left(f^{2}+3 g^{2}\right)-\frac{1}{M} \Lambda\right] \\
\Lambda=4\left(f+f_{2}\right) g\left(2 E_{e}^{(0)}+\Delta\right)-4 \Delta g^{2}+4\left(E_{e}^{(0)}+\Delta\right)\left(f^{2}+5 g^{2}\right)
\end{array}
$$

com os parâmetros:

$$
\begin{array}{r}
f=1 \\
f_{2}=3,706 \\
g=1,26 \\
\theta_{C}=0,974 \\
\sigma_{0}=1,024 \frac{G_{F}^{2} \cos ^{2} \theta_{C}}{\pi} .
\end{array}
$$

Na Fig. (4.3) apresentamos o comportamento dessa seção de choque em função da energia do neutrino.

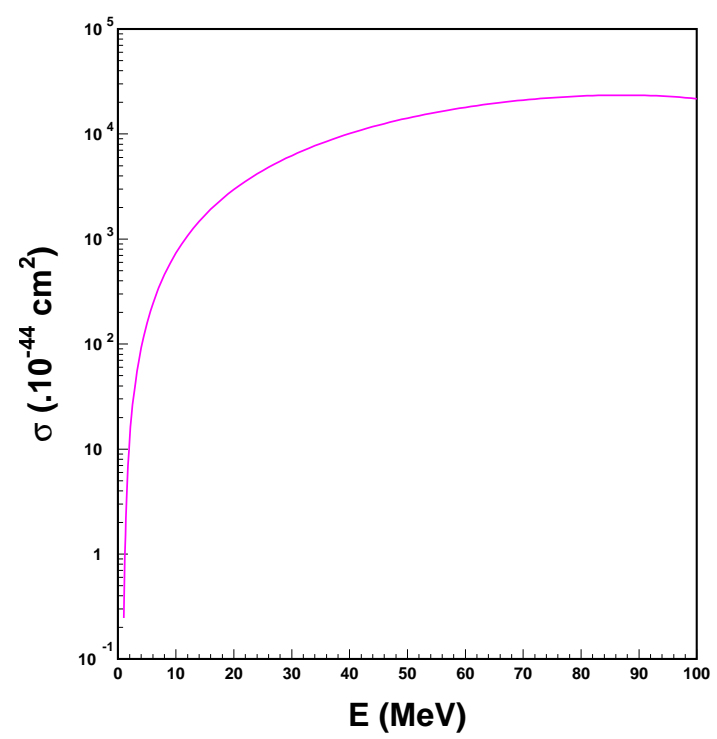

Figura 4.3: Seção de choque para o decaimento beta inverso até primeira ordem de teoria de perturbação [144].

Assumimos uma eficiência $(\epsilon)$ de $100 \%$ acima do limiar de energia de $5 \mathrm{MeV}$. Este percentual foi considerado por estarmos simulando possíveis experimentos que ainda não possuem uma eficiência 
característica conhecida. A distribuição energética e o número total de antineutrinos do elétron detectados na Terra, $d N / d E$ e $N_{T O T}$, podem ser calculados como

$$
\begin{gathered}
d N / d E\left(\left\langle E_{e}\right\rangle,\left\langle E_{\bar{e}}\right\rangle,\left\langle E_{x}\right\rangle, E_{b} / D^{2}, \operatorname{sen}^{2} \theta_{13},\left|\Delta m_{31}^{2}\right|\right)=n_{p} \epsilon \sigma(E) \int_{t} F_{\overline{\nu_{e}}}(E, t) d t, \\
N_{T O T}\left(\left\langle E_{e}\right\rangle,\left\langle E_{\bar{e}}\right\rangle,\left\langle E_{x}\right\rangle, E_{b} / D^{2}, \operatorname{sen}^{2} \theta_{13},\left|\Delta m_{31}^{2}\right|\right)=n_{p} \epsilon \int_{E} \int_{t} F_{\overline{\nu_{e}}}(E, t) \sigma(E) d E d t,
\end{gathered}
$$

sendo $n_{p}$ o número de prótons no volume fiducial do detector Cherenkov e ficando implicita a dependência do fluxo com parâmetros de SN e oscilação. O intervalo de tempo $t$ considerado para observação foi de 12 segundos. Para tempos superiores o número de neutrinos e o fluxo caem substancialmente de forma que praticamente não serão identificáveis. Pelo mesmo motivo, o intervalo de energia considerado foi de $5 \mathrm{MeV}$ até $80 \mathrm{MeV}$.

Os dados foram simulados para detectores Cherenkov, que tem obtido bastante sucesso nesse tipo de medição. Existe uma proposta de substituição do detector Super-Kamiokande por um detector maior, Hyper-Kamiokande [145], que terá uma massa em torno de uma megatonelada. Além desta, existem outras propostas bastante semelhantes como o detector UNO, e o MEMPHYS [146, 147], todos com sensibilidade semelhante. Assumiremos aqui um volume efetivo de 540 kton e detecção através do decaimento beta inverso.

\subsection{Dependência do número de eventos com os parâmetros da su- pernova}

Uma vez calculado o espectro esperado, é possível simular espectros experimentais para valores determinados dos parâmetros de oscilação de SN. Assim, será possível estudar a capacidade do detector de determinar alguns destes parâmetros.

A partir do espectro obtido são definidos intervalos de energia e, para cada um, é calculado um $\chi^{2}$ de forma que possamos definir intervalos de confiança.

A função $\chi^{2}$ é construida de forma a obtermos um limite na incerteza experimental com relação as variáveis associadas ao valor teórico da função resposta. No caso em estudo esta função pode ser tanto o número total de eventos $N_{T O T}$ quanto sua variação na energia $d N / d E$. Assim, a função resposta será:

$$
\begin{gathered}
d N / d E_{T O T}^{\text {teor }}=d N / d E^{\text {teor }}\left(\operatorname{sen}^{2} \theta_{13},\left\langle E_{\bar{e}}\right\rangle,\left\langle E_{x}\right\rangle, E_{b} / D^{2}, \operatorname{sign}\left|\Delta m_{31}^{2}\right|\right) . \\
N_{T O T}^{\text {teor }}=N^{\text {teor }}\left(\operatorname{sen}^{2} \theta_{13},\left\langle E_{\bar{e}}\right\rangle,\left\langle E_{x}\right\rangle, E_{b} / D^{2}, \operatorname{sign}\left|\Delta m_{31}^{2}\right|\right) .
\end{gathered}
$$

O cálculo do $\chi^{2}$ é feito em cada intervalo de energia $d E$ do espectro 


$$
\chi_{i}^{2}=\frac{\left(N_{i}^{\text {teor }}\left(\left\langle E_{\bar{e}}\right\rangle,\left\langle E_{x}\right\rangle\right)-N_{T O T i}^{e x p}\right)^{2}}{N_{i}^{\text {teor }}\left(\left\langle E_{\bar{e}}\right\rangle,\left\langle E_{x}\right\rangle\right)},
$$

e posteriormente é somado de forma a fornecer uma região de incerteza em torno do ponto teórico

$$
\chi^{2}=\sum_{i} \chi_{i}^{2}
$$

Desta forma, definidos os parâmetros de SN que entrarão como variáveis no cálculo do número de eventos experimental, obteremos como este número de eventos depende de cada um dos parâmetros.

\subsection{Casos estudados}

Tendo em vista o desenvolvimento feito até aqui, é possível simular o espectro e o número de eventos esperado na Terra vindos de uma SN galática. O intervalo de tempo e energia observados, além do detector proposto, estão descritos em seções anteriores. Vamos considerar que os parâmeros de SN estejam nos intervalos previstos pelas simulações [148].

$$
\begin{gathered}
<E_{e}>\in[9-15] \mathrm{MeV}, \\
<E_{\bar{e}}>\in[12-17] \mathrm{MeV}, \\
<E_{x}>\in[15-30] \mathrm{MeV}, \\
E_{b} / D^{2} \in(2,0-4,0) \times 10^{51} \mathrm{ergs} / \mathrm{kpc}^{2} .
\end{gathered}
$$

Na Tabela 4.1 definimos um ponto no espaço de parâmetros de SN para ser utilizado como referência.

\begin{tabular}{|c|c|c|c|}
\hline$<E_{e}>(\mathrm{MeV})$ & $<E_{\bar{e}}>(\mathrm{MeV})$ & $<E_{x}>(\mathrm{MeV})$ & $E_{b} / D^{2}\left(\mathrm{ergs} / \mathrm{kpc}^{2}\right)$ \\
\hline 12 & 15 & 18 & $3,0 \times 10^{51}$ \\
\hline
\end{tabular}

Tabela 4.1: Parâmetros da SN utilizados para as simulações.

Vamos estudar aqui apenas dois casos extremos para valores de $\theta_{13}$ (Fig. 4.1). Estes dois extremos correspondem a $P_{H}=0$ e $P_{H}=1$, além de considerar as duas hierarquias possíveis. Desta forma, teremos quatro cenários possíveis:

1. Hierarquia inversa e $P_{H} \simeq 0\left(\operatorname{sen}^{2} \theta_{13}=10^{-3}, \theta_{13}\right.$ grande $)$;

2. Hierarquia normal e $P_{H} \simeq 0\left(\operatorname{sen}^{2} \theta_{13}=10^{-3}, \theta_{13}\right.$ grande $)$;

3. Hierarquia inversa e $P_{H} \simeq 1\left(\operatorname{sen}^{2} \theta_{13}=10^{-6}, \theta_{13}\right.$ pequeno);

4. Hierarquia normal e $P_{H} \simeq 1\left(\operatorname{sen}^{2} \theta_{13}=10^{-6}, \theta_{13}\right.$ pequeno $)$. 

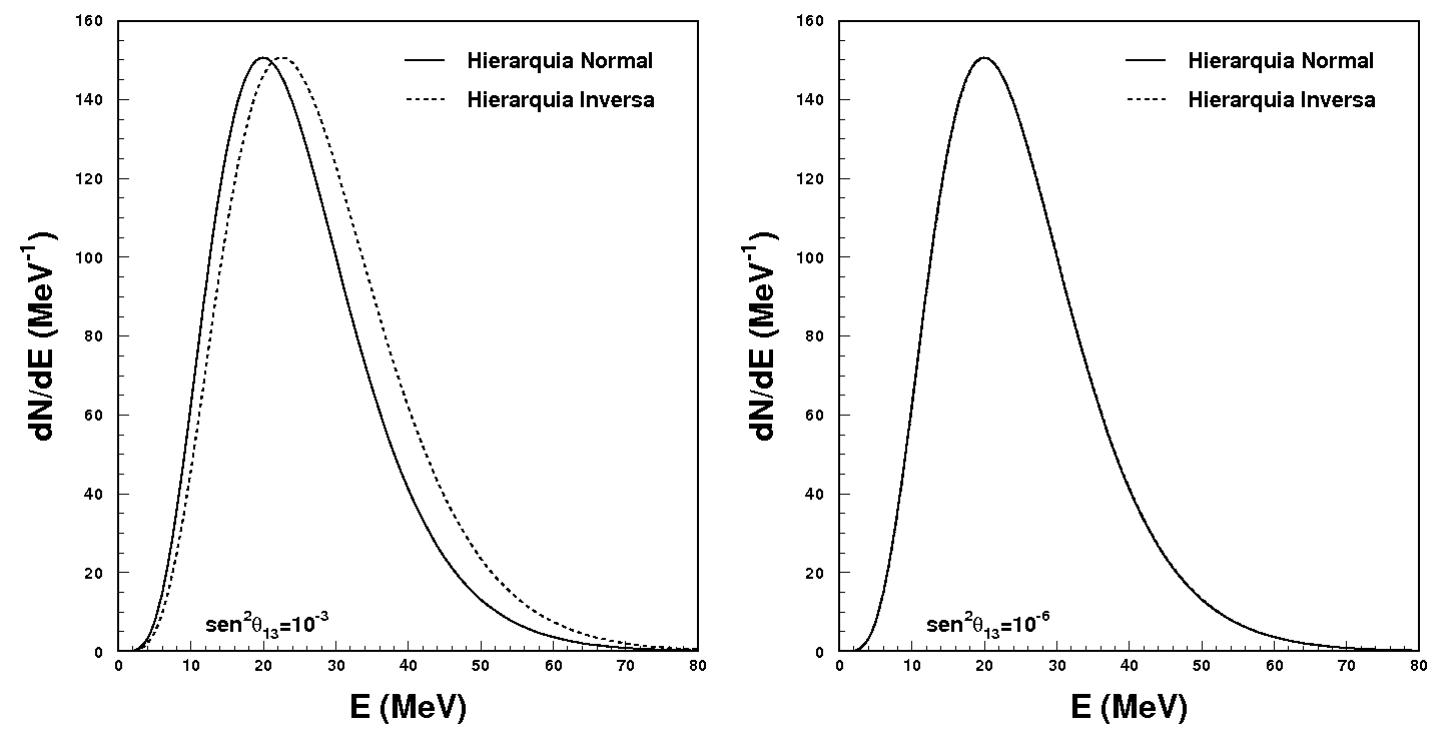

Figura 4.4: Espectro de energia de antineutrinos que se espera observar em um detector Cherenkov de 540 kton e eficiência de $100 \%$ para observação do neutrino através da reação de decaimento beta inverso no intervalo de tempo de $6 \pm 0,25 \mathrm{~s}$. O gráfico a esquerda corresponde ao ângulo de mistura $\operatorname{sen}^{2} \theta_{13}=10^{-3}$ e o gráfico a direita a $\operatorname{sen}^{2} \theta_{13}=10^{-6}$. Ambos mostram os casos de hierarquia normal e inversa.

O espectro ne neutrinos esperado para cada um dos casos pode ser visto na Fig. (4.4), enquanto os resultados obtidos para o número de eventos encontram-se na Tabela 4.2.

\begin{tabular}{|c|c|}
\hline Caso & $N_{T O T}$ \\
\hline$\theta_{13}$ grande e HI & 178151 \\
\hline$\theta_{13}$ grande e HN & 160969 \\
\hline$\theta_{13}$ pequeno e HI & 161053 \\
\hline$\theta_{13}$ pequeno e HN & 160952 \\
\hline
\end{tabular}

Tabela 4.2: Número esperado de eventos de supernova em um detector Cherenkov de 540 kton para reação de decaimento beta inverso para o ponto no espaço de parâmetros definido na Tabela (4.1).

Os resultados obtidos para os casos 3 e 4 ( $\theta_{13}$ pequeno) devem ser estatisticamente iguais pois, tendo $P_{H} \simeq 1, P_{\bar{e} \bar{e}}(\mathrm{HN}) \simeq P_{\bar{e} \bar{e}}(\mathrm{HI})$ (Eqs. (4.6) - (4.8)), como mostra a Fig.(4.4) e a tabela (4.2). Desta forma, podemos considerar que, para o caso em estudo, os resultados para o ângulo $\theta_{13}$ pequeno independem da hierarquia. 


\subsection{Estudo preliminar da dependência do número total de eventos com os parâmetros de supernova}

Após obtermos a distribuição em energia dos eventos correspondentes a antineutrinos para valores fixos dos parâmetros, podemos agora analisar como esse número de eventos varia quando fixamos todos os parâmetros com excessão de um deles.
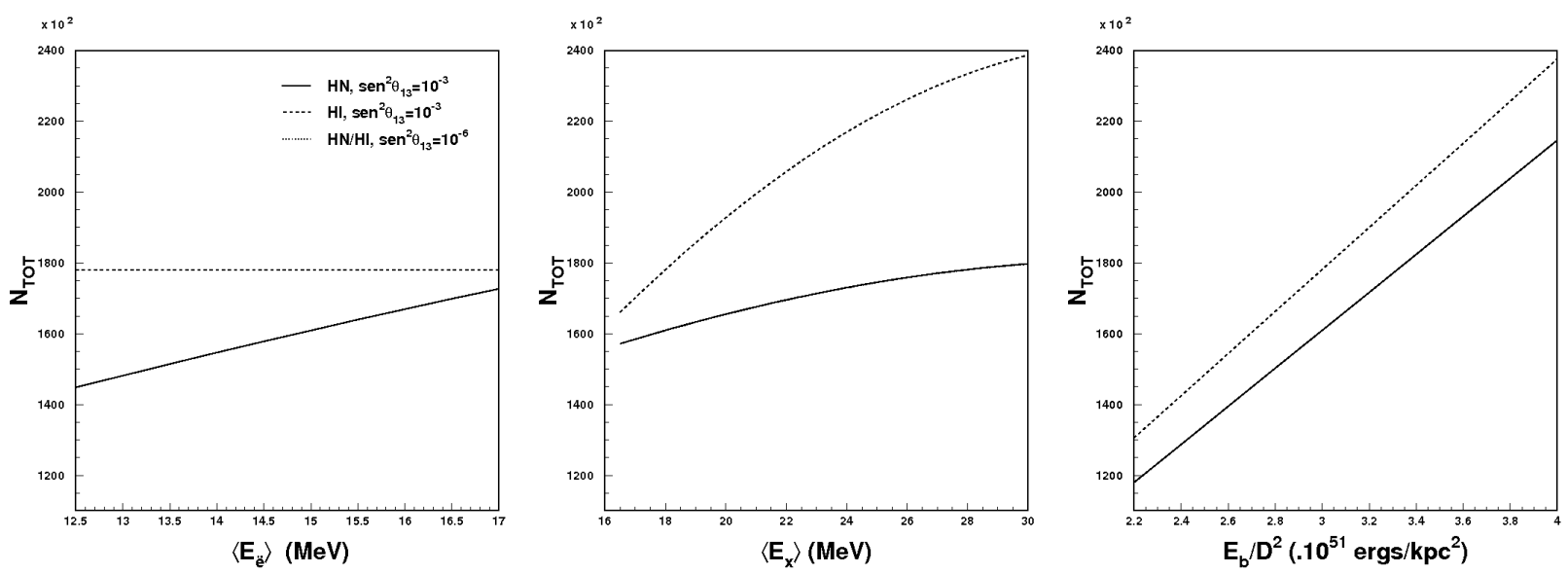

Figura 4.5: Variação do número de eventos $N_{T O T}$ como função das energias médias do antineutrino do elétron dos neutrinos não-eletrônicos e de $E_{b} / D^{2}$, respectivamente. Estamos considerando hierarquia normal e ângulo de mistura grande e os parâmetros da SN fixos segundo a Tabela (4.1).

Em primeiro lugar notamos que a dependência do número total de eventos com as energias médias e $E b / D^{2}$ é exatamente igual para o caso de $\mathrm{HN}$ com ângulo de mistura grande ou pequeno. Isto acontece porque nestes casos a probabilidade de sobrevivência $P_{\bar{e} \bar{e}}$ é idêntica, Eq. (4.1), gerando um fluxo 4.10 de antineutrinos eletrônicos equivalente. Esta igualdade será mantida ao longo de toda esta análise.

Outra observação importante refere-se ao $N_{T O T}$ constante para qualquer $<E_{\bar{e}}>$ no caso de HI e ângulo de mistura grande. Uma vez que neste ponto $P_{\bar{e} \bar{e}}=0$, o fluxo final não terá dependência com o fluxo de $\bar{\nu}_{e}$, de forma que sua energia média não entre como variável. Nos demais casos, o número total de eventos $N_{T O T}$ terá seu comportamento de acordo com a Eq. (4.4).

A partir dos dados simulados é possível obter regiões de maior probabilidade para cada um destes parâmetros. Para tal, vamos dividir os eventos em intervalos de energia de $10 \mathrm{MeV}$. Para cada intervalo de energia com número de eventos $N_{i}$, a incerteza estatística associada é $\sqrt{N_{i}}$. 

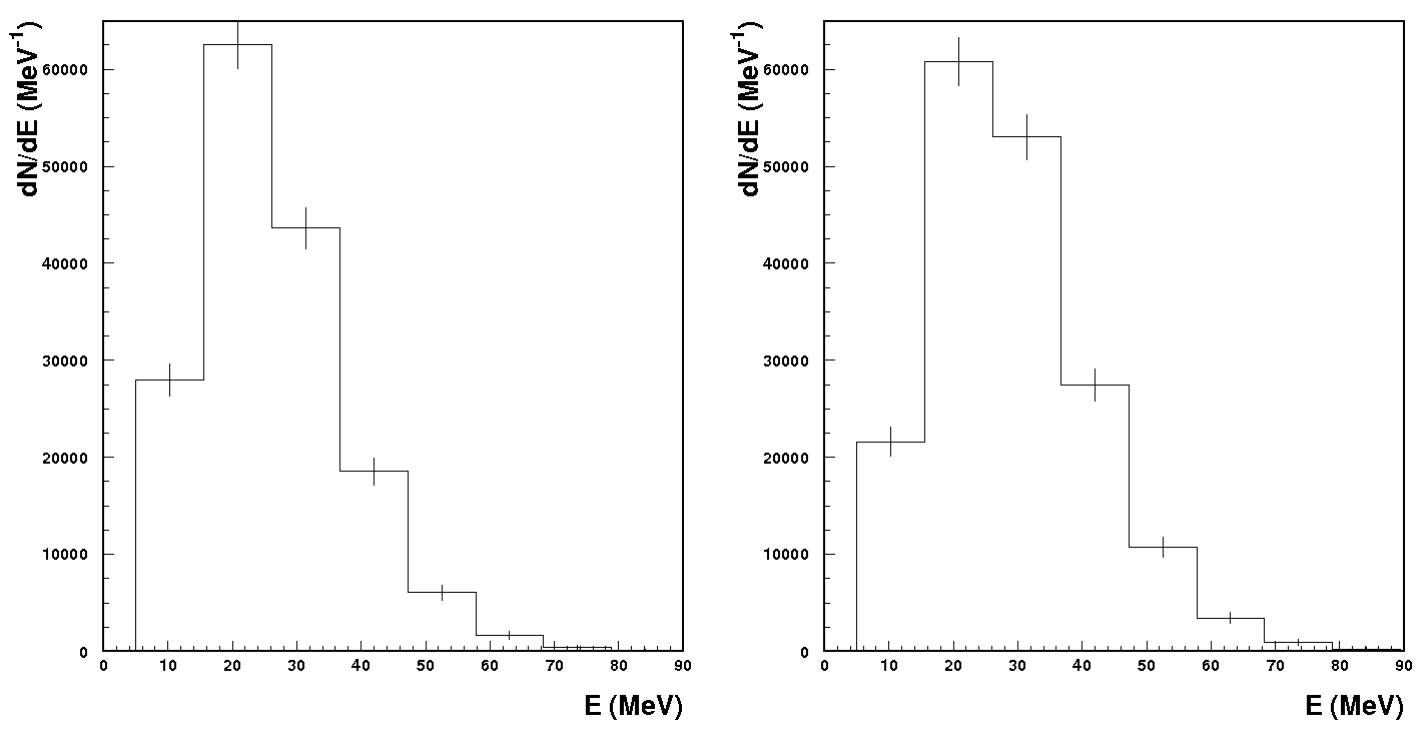

Figura 4.6: Distribuição do número de eventos de $\bar{\nu}_{e}$ para intervalos de energia de $10 \mathrm{MeV}$. O primeiro caso corresponde a ângulo de mistura grande, $\operatorname{sen}^{2} \theta_{13}=10^{-3}$, e HN (caso equivalente a ângulo de mistura pequeno, $\operatorname{sen}^{2} \theta_{13}=10^{-6}$. O segundo caso se refere tambem a ângulo de mistura grande com HI, A barra de erro apresentada refere-se apenas à incerteza estatística multiplicada por 10. Os parâmetros fixos são os mesmos da Fig. (4.5)

A soma do $\chi^{2}$ associado a cada intervalo fornece a dependência do número de eventos para cada par de parâmetros que caracterizam a SN. Caso um detector realize uma medida $N_{T O T} \pm \sqrt{N_{T O T}}$ eventos, tornará possível limitar os valores destes parâmetros. Vamos considerar inicialmente os parâmetros de SN fixos nos valores da tabela (4.1) e calcular a variação de pares destes parâmetros, posteriormente graficados. Segue, nas figuras, a região permitida do plano em $1 \sigma, 2 \sigma$ e $3 \sigma$, para cada caso. 

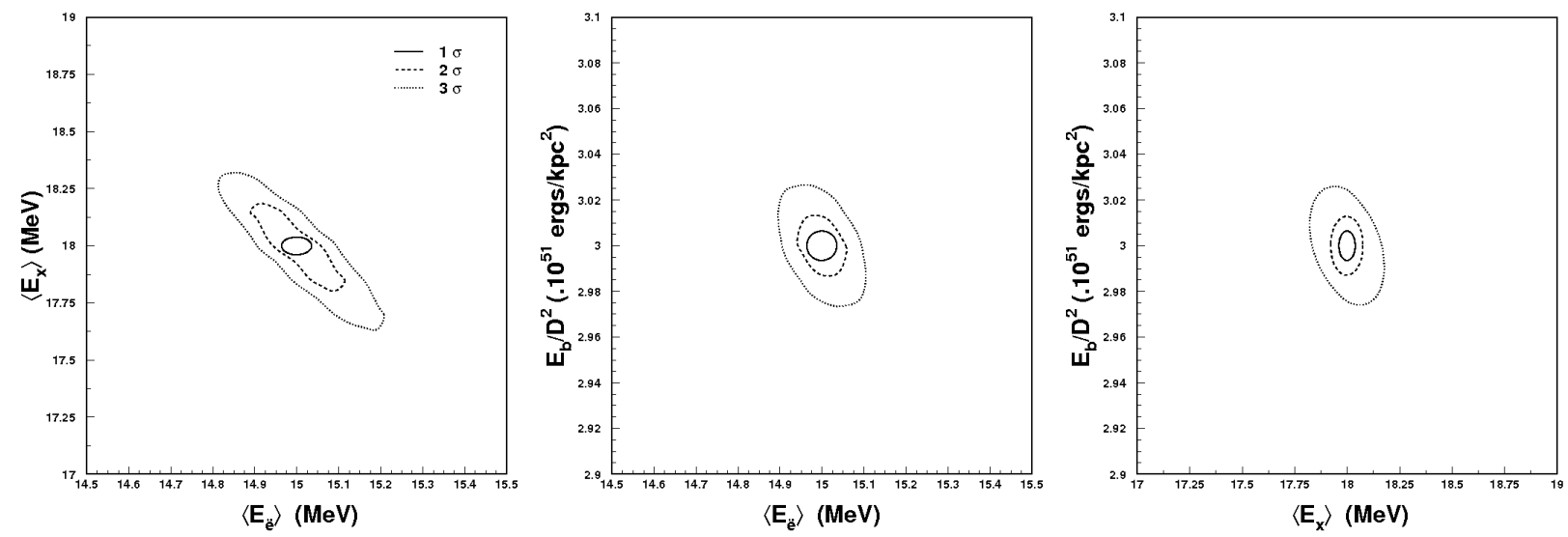

Figura 4.7: Sensibilidade do detector para os parâmetros $\left\langle E_{\bar{e}}\right\rangle,\left\langle E_{x}\right\rangle$ e $E_{b} / D^{2}$ de supernova dois a dois em hierarquia normal e ângulo de mistura grande, $\operatorname{sen}^{2} \theta_{13}=10^{-3}$

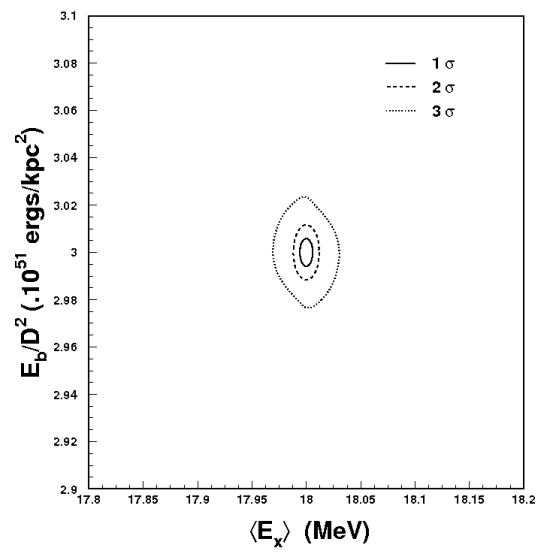

Figura 4.8: Sensibilidade do detector para os parâmetros $\left\langle E_{x}\right\rangle$ e $E_{b} / D^{2}$ de supernova em hierarquia inversa e ângulo de mistura grande, $\operatorname{sen}^{2} \theta_{13}=10^{-3}$

Como já citamos, o caso de ângulo de mistura pequeno é equivalente ao de hierarquia normal e ângulo de mistura grande. Desta forma, consideramos ambos como mostra a Fig. (4.7). Como vimos na Fig. (4.5), o número de eventos não varia com $\left\langle E_{\bar{e}}\right\rangle$ no caso de HI e ângulo de mistura grande. Desta forma, não existe sensibilidade da detecção com relação a este parâmetro. É possível observar um limite apenas com relação a $\left\langle E_{x}\right\rangle$ e $E_{b} / D^{2}$, visto na Fig. (4.8). 


\subsection{Estudo da avaliação da precisão esperada para determinação dos parâmetros de supernova}

Agora o cálculo do $\chi^{2}$ será feito com a variação dos 3 parâmetros de forma a refinar o resultado anteriormente apresentado. Usamos novamente intervalos de energia de $10 \mathrm{MeV}$ cada a partir de 5 $\mathrm{MeV}$. Este cálculo foi feito buscando o valor mínimo do $\chi^{2}$ na variação da dimensão suprimida no gráfico.
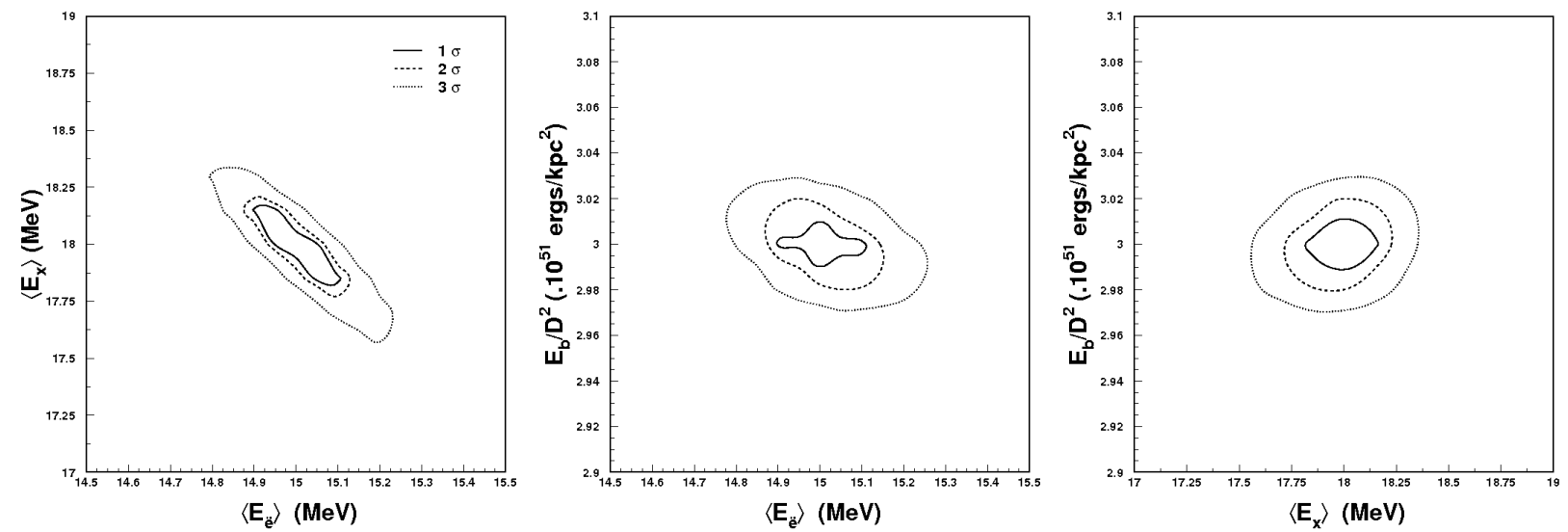

Figura 4.9: Sensibilidade do detector para os parâmetros $\left\langle E_{\bar{e}}\right\rangle,\left\langle E_{x}\right\rangle$ e $E_{b} / D^{2}$ de supernova em hierarquia normal e ângulo de mistura grande, $\operatorname{sen}^{2} \theta_{13}=10^{-3}$

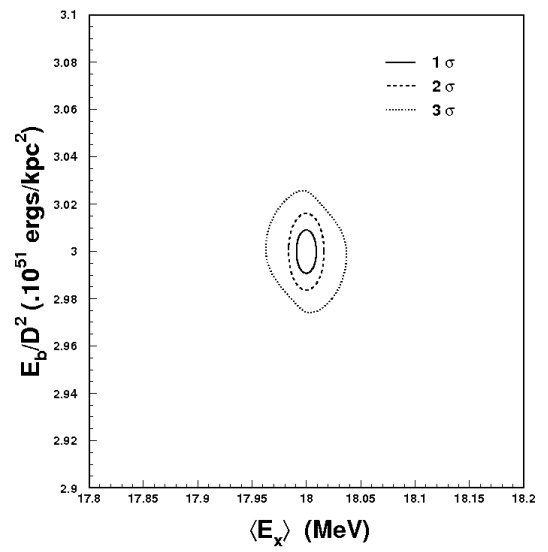

Figura 4.10: Sensibilidade do detector para os parâmetros $\left\langle E_{x}\right\rangle$ e $E_{b} / D^{2}$ de supernova em hierarquia inversa e ângulo de mistura grande, $\operatorname{sen}^{2} \theta_{13}=10^{-3}$

Novamente não haverá dependência com $\left\langle E_{\bar{e}}\right\rangle$ para hierarquia inversa. Em todos os outros casos mostrados, é possível definir limites inferiores e superiores dos parâmetros, se considerarmos que os 
parâmetros de oscilação tenham já sido definidos por experimentos. Vemos também que a sensibilidade em $\left\langle E_{x}\right\rangle$ é muito mais forte que nos demais casos, foenecendo uma maior precisão neste parâmetro. 


\section{Capítulo 5}

\section{Estudo do efeito de uma onda de choque em supernovas galáticas no espectro dos neutrinos observados na Terra}

Uma onda de choque representa uma maior perturbação na densidade da pré-supernova e também na fração de elétrons $\left(Y_{e}\right)$ no meio. Esta onda aparece na região acima da estrela de nêutrons, onde os neutrinos têm energia típica de $E_{\nu} \approx 10 \mathrm{MeV}$ e terão ressonância por volta de $t_{p b} \approx 0,1-15 \mathrm{~s}$. É neste período que o fluxo de neutrinos será grande o suficiente potencializando a sua detecção por um experimento terrestre [77].

Uma vez que a frente de onda é bem acentuada, a conversão de neutrinos pode ser interrompida enquanto essa frente passa pela região de ressonância MSW. Estes sinais podem ser detectados na Terra através de distorções no espectro de neutrinos em detectores terrestres.

O cálculo anteriormente feito será repetido agora considerando uma supernova de potencial variável conforme a passagem de uma onda de choque, com o objetivo de verificar se, para a sensibilidade dos detectores sob estudo, este refinamento será ou não relevante.

\subsection{Perfil do potencial}

Novamente, o valor referente ao $\operatorname{sen}^{2} \theta_{21}$ será, em primeira aproximação, irrelevante para neutrinos de supernova pois correspondem a transições não observáveis. Desta forma, trabalharemos com transições de ressonância H, com número de onda segundo a Eq. (3.20).

Os efeitos de matéria se potencializarão quando $k_{H}$ tiver valores próximos ao potencial $\mathrm{V}$ (Eq. (1.54)), sendo este descrito pela Eq. (3.2), que em unidades apropriadas fica:

$$
\frac{V(x)}{\mathrm{eV}^{2} / \mathrm{MeV}}=3,8 \times 10^{-8} \frac{\rho(x)}{\mathrm{g} / \mathrm{cm}^{3}}
$$

sendo $\rho(x)$ a densidade radial de massa. 
Como já vimos, este potencial é aproximado pela lei de potencia $V(x) \approx x^{-3}$. Contudo, na presença da onda de choque, este perfil estático será modificado [149]. A onda de choque, enquanto se propaga a velocidade supersônica, deixa uma zona de rarefação caracterizada por uma região de alta densidade e outra de densidade abaixo da estática. O ponto de descontinuidade no potencial será o raio da frente de onda, $x_{s}$. Definimos os seguintes potenciais:

$$
\begin{aligned}
V^{+} & =\lim _{x \rightarrow x_{s}^{-}} V(x), \\
V^{-} & =\lim _{x \rightarrow x_{s}^{+}} V(x),
\end{aligned}
$$

com uma razão típica

$$
\epsilon=\frac{V^{+}}{V^{-}} \cong 10
$$

Devido a esta descontinuidade, discutiremos expressões analíticas para a probabilidade de transição nas posições $x=x_{s}$ e $x \neq x_{s}$ separadamente.

\subsection{Parametrização empírica do perfil de densidades}

Apresentaremos aqui uma parametrização empírica do perfil de densidades $\rho(x)$ que reproduz o potencial proposto [149], Fig. (5.1), sendo contínuo em $x$ e $t$, com excessão do ponto $x(t)=x_{s}(t)$.

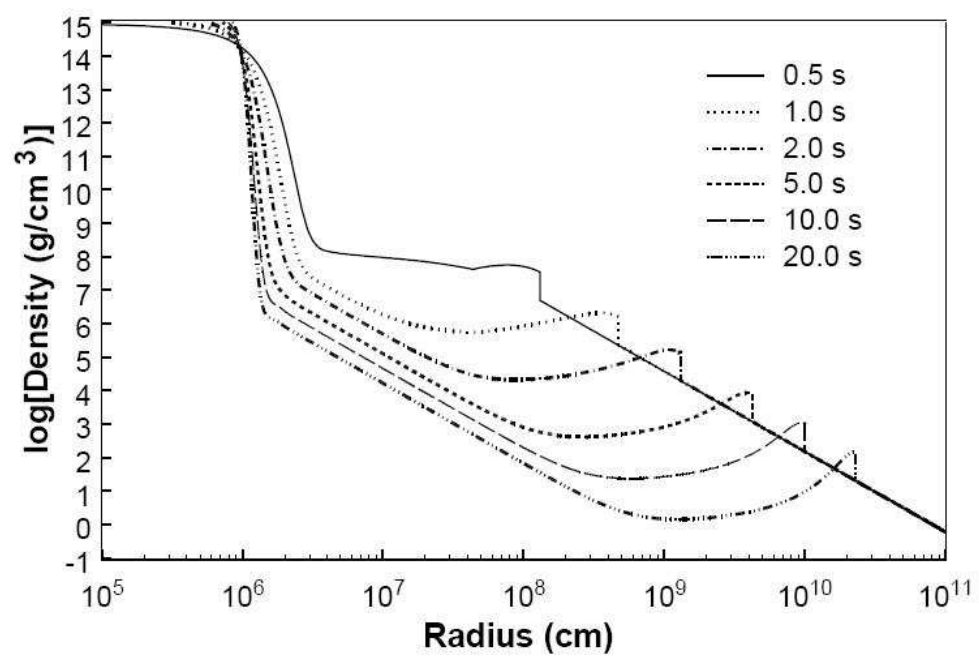

Figura 5.1: Perfil de densidade simulado para uma supernova com onda de choque como função do raio para diferentes tempos [149] 
Para tempos $t<1 \mathrm{~s}$ após a explosão, os efeitos da onda de choque $[149,150]$ aparecerão apenas para $V>k_{H}$ e , portanto, não afetarão o sub-sistema H. Este potencial poderá ser aproximado no limite estático como [149]

$$
t<1 \mathrm{~s} \rightarrow \frac{\rho_{0}(x)}{\mathrm{g} / \mathrm{cm}^{3}} \simeq 10^{14}\left(\frac{x}{\mathrm{~km}}\right)^{-2,4} .
$$

Com a passagem da onda de choque este potencial será descrito em termos do ponto da frente de onda $x_{s}$ e da perturbação $f(x)$ que esta causará ao perfil, apenas para $x \leq x_{s}$.

$$
t>1 \mathrm{~s} \rightarrow \rho(x) \cdot \begin{cases}\epsilon \cdot f(x) & , x \leq x_{s} \\ 1 & , x>x_{s}\end{cases}
$$

com $\epsilon$ definido na Eq. (5.4).

Nesta equação, a função $f(x)$ parametriza a zona de rarefação após a passagem da onda de choque. Esta parametrização pode ser definida como [143]

$$
\ln f(x)=\left[0,28-0,69 \ln \left(x_{s} / \mathrm{km}\right)\right] \cdot\left[\arcsin \left(1-x / x_{s}\right)\right]^{1,1} .
$$

Assuminos que a evolução da posição da frente de onda no tempo será a seguinte:

$$
x_{s}(t)=x_{s}^{0}+v_{s} t+\frac{1}{2} a_{s} t^{2},
$$

com parâmetros aproximadamente dados por:

$$
\begin{gathered}
x_{s}^{0}=-4,6 \times 10^{3} \mathrm{~km} \\
v_{s}=11,3 \times 10^{3} \mathrm{~km} / \mathrm{s} \\
a_{s}=0,2 \times 10^{3} \mathrm{~km} / \mathrm{s}^{2} .
\end{gathered}
$$

O potencial referente a parametrização escolhida [ Eqs. (5.5) - (5.9)] segue no gráfico (5.2). 


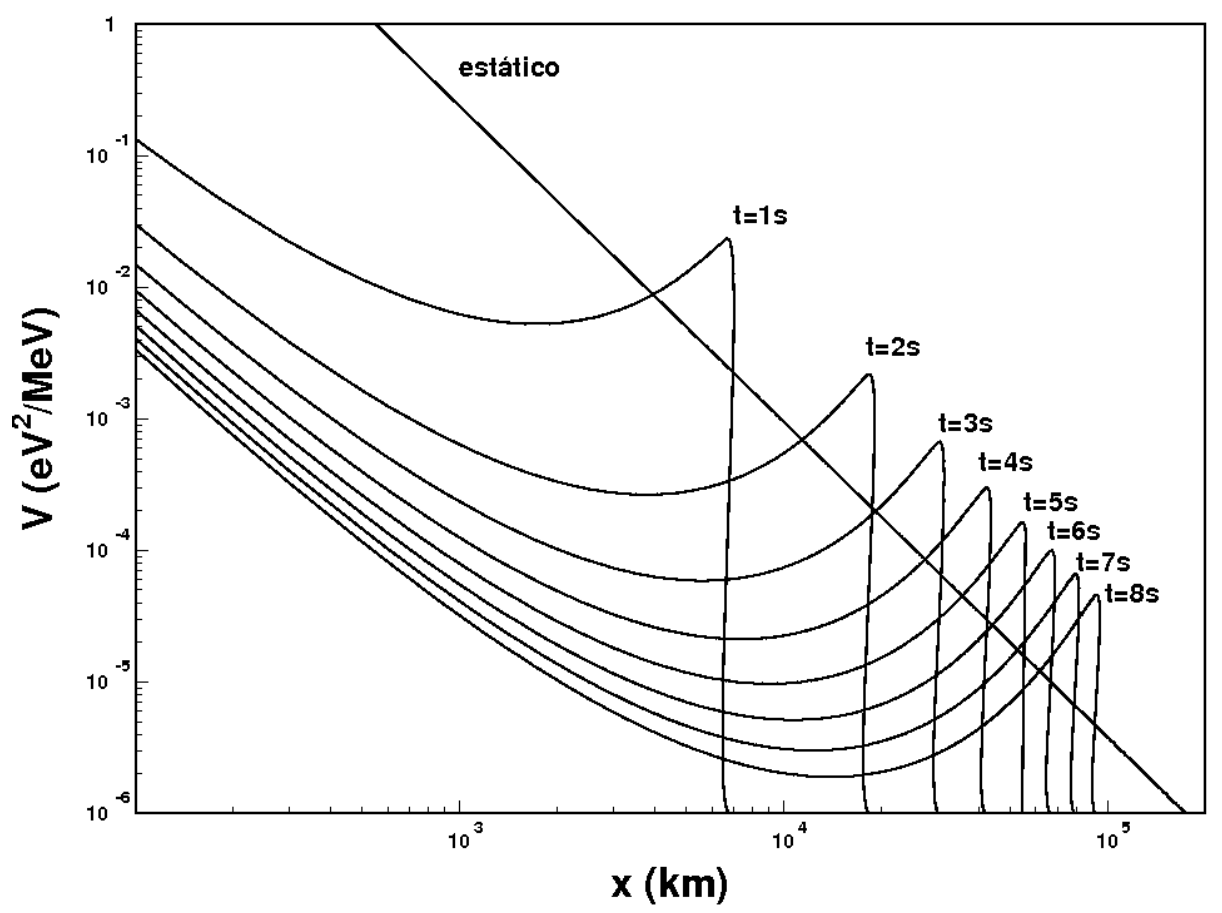

Figura 5.2: Potencial de matéria em função da distância radial do centro da supernova para tempos diferentes $(\mathrm{t}=1-8 \mathrm{~s}$ de cima para baixo). É fácil perceber que podem existir até três pontos com o mesmo potencial $\mathrm{V}$, pois há uma queda no potencial no ponto em que se encontra a frente de onda.

Neste potencial haverá três pontos que satisfazem as condições de ressonância. Dois deles correspondem à região de rarefação $\left(x_{1}<x_{2}<x_{s}\right)$, sendo $x_{s}$ o raio da frente de onda que evolui com o tempo e outro à região estática $\left(x_{3}>x_{s}\right)$. Estes três pontos, mais a frente de onda, devem ser levados em consideração no cálculo da probabilidade de transição [143].

Uma descrição detalhada do potencial $V(x)$ na frente de onda é muito pouco conhecido, sendo que a descontinuidade em $x=x_{s}$ é colocada a mão como correção aos potenciais numéricos em que há uma continuidade suave neste ponto.

Além disto, é esperado que haja uma turbulência na zona de rarefação após a passagem da frente de onda, induzindo variações na densidade local e levando a mais que três soluções de condição de ressonância em alguns casos. Estes pontos não foram levados em consideração no trabalho mas devem ser avaliados em simulações mais refinadas.

\subsection{Probabilidades de transição}

a) Para $x \neq x_{s}$

Quando a condição 


$$
V\left(x_{H}\right)=k_{H},
$$

for satisfeita, haverá um maior efeito de matéria no ponto $x_{H}$, e uma probabilidade finita $P_{H}$ de transição entre os autoestados de massa do subsistema H. Esta probabilidade é dada em boa aproximação pela Eq. (3.11):

$$
P_{H}=\frac{\exp \left(2 \pi r_{H} k_{H} \cos ^{2} \theta_{13}\right)-1}{\exp \left(2 \pi r_{H} k_{H}\right)-1}
$$

para neutrinos e antineutrinos [106] com

$$
r_{H}=\left|\frac{d \ln V(x)}{d x}\right|_{x=x_{H}}^{-1} .
$$

Para os potenciais $V(x)$ e valores de $\operatorname{sen}^{2} \theta_{13}$ utilizados nesta dissertação, a equação se reduz ao limite de Landau-Zener

$$
P_{H} \simeq-\exp \left(2 \pi r_{H} k_{H} \operatorname{sen}^{2} \theta_{13}\right)
$$

Em princípio a condição $V\left(x_{L}\right)=k_{L}$ poderia também acontecer para algum ponto da supernova. Contudo, para o ângulo de mistura solar compatível com os resultados experimentais, $\operatorname{sen}^{2} \theta_{12}=$ 0,315, estas transições tornam-se adiabáticas, ou seja,

$$
P_{L}(\nu) \simeq 0 \simeq P_{L}(\bar{\nu})
$$

para todo $x \neq x_{s}$.

b) Para $x=x_{s}$

Neste ponto, a probabilidade de transição será determinada pela conservação de sabor através da descontinuidade de $V(x)$ [151]

$$
P_{s}=\operatorname{sen}^{2}\left(\theta_{m}^{+}-\theta_{m}^{-}\right)
$$

sendo $\theta_{m}^{+}$e $\theta_{m}^{-}$os ângulos de mistura efetivos na matéria imediatamente antes $\left(V=V^{+}\right)$e depois $\left(V=V^{-}\right)$da frente de onda. Estes ângulos são definidos, para transições $\mathrm{H}$,

$$
\cos 2 \theta_{m}^{ \pm}=\frac{\cos 2 \theta_{13}-V^{ \pm} / k_{H}}{\sqrt{\left(\cos 2 \theta_{13}-V^{ \pm} / k_{H}\right)^{2}+\left(\operatorname{sen} 2 \theta_{13}\right)^{2}}}
$$

para neutrinos e antineutrinos. Para $\theta_{13}$ pequenos, $P_{s} \simeq 1$ para $k_{H} \in\left[V_{-}, V_{+}\right]$e $P_{s} \simeq 0$ nos outros casos. 
A probabilidade de transição no setor L pode ser facilmente obtida pelas substituições $\theta_{13} \rightarrow \theta_{12} \mathrm{e}$ $k_{H} \rightarrow k_{L}$. O caráter não adiabático destas transições podem levar a um $P_{L} \neq 0$ em alguns intervalos do espaço de parâmetros. Contudo terão pouca relevância fenomenológica.

\section{c) Expressão analítica para múltiplas transições}

Para o potencial estudado, a condição de ressonância pode ser satisfeita em até três pontos do interior da supernova, isto é

$$
V\left(x_{i}\right)=k_{H}(i=1,2,3) .
$$

No caso mais geral, dois destes pontos pertencem a zona de rarefação $\left(x_{1}<x_{2}<x_{s}\right)$ e um à região de potencial estático, em raios maiores que o da frente de onda $\left(x_{3}>x_{s}\right)$. Além destes, temos a probabilidade de transição relativa ao ponto de descontinuidade $x_{s}$. Assim, as probabilidades de transição serão $P_{1}, P_{2}, P_{s}$ e $P_{3}$, para os pontos $x_{1}<x_{2}<x_{s}<x_{3}$ respectivamente.

Assumindo que pode ser feita uma média com a contribuição de cada uma, a probabilidade de transição geral pode ser definida pela equação matricial

$$
\left(\begin{array}{cc}
1-P_{H} & P_{H} \\
P_{H} & 1-P_{H}
\end{array}\right)=\prod_{i=1,2, s, 3}\left(\begin{array}{cc}
1-P_{i} & P_{i} \\
P_{i} & 1-P_{i}
\end{array}\right)
$$

que tem como solução

$$
\begin{array}{r}
P_{H}=P_{1}+P_{2}+P_{s}+P_{3}-2\left(P_{1} P_{2}+P_{1} P_{s}+P_{1} P_{3}+P_{2} P_{s}+P_{2} P_{3}+P_{s} P_{3}\right)+ \\
4\left(P_{1} P_{2} P_{s}+P_{1} P_{2} P_{3}+P_{1} P_{s} P_{3}+P_{2} P_{s} P_{3}\right)-8 P_{1} P_{2} P_{s} P_{3} .
\end{array}
$$

$\mathrm{Na}$ análise da probabilidade de transição final podem aparecer alguns pontos críticos nos casos:

(1) atrás da zona de rarefação $\left(x_{r}\right)$ quando $x_{1}=x_{2}$,

(2) na frente de onda $\left(x_{s}\right)$ quando $x_{2}=x_{s}$ para $V^{+}=k_{H}$ ou $x_{3}=x_{s}$ para $V^{-}=k_{H}$.

Estes pontos serão limítrofes do intervalo de contribuição das probabilidades dominantes, de forma que $P_{H}$ seja contínua e terão grande importância na forma final da probabilidade de transição, como veremos na próxima seção.

\subsubsection{Dependência energética da probabilidade de transição}

Na Fig.(5.3) mostramos o resultado do cálculo da probabilidade de transição $P_{H}(E)$ para cinco valores diferentes de $\operatorname{sen}^{2} \theta_{13}$ em um tempo fixo $t=4 \mathrm{~s}$ após a explosão. Em cada figura aparece também a contribuição particular de cada $P_{i}(E)(i=1,2, s, 3)$. 

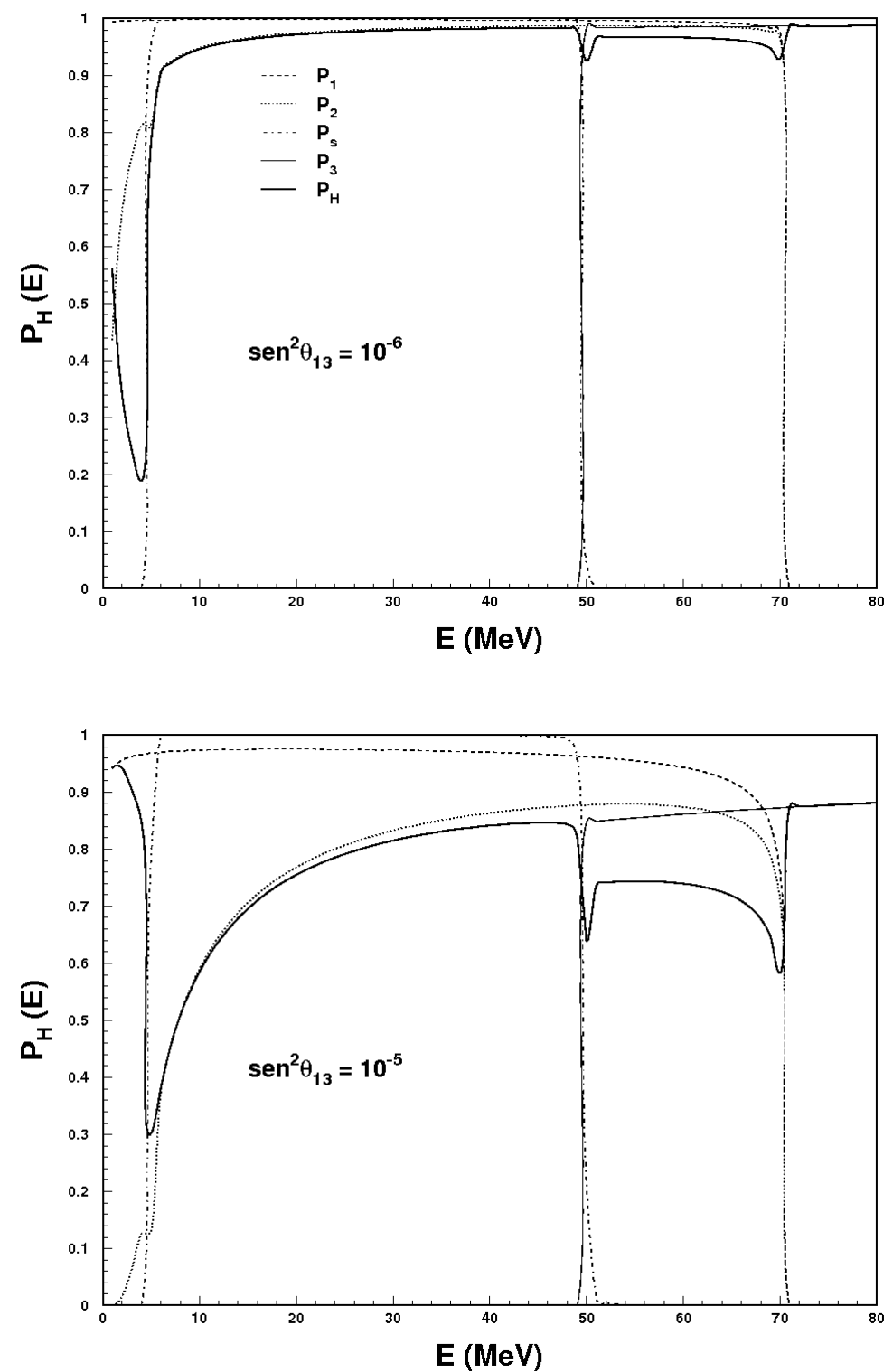

Figura 5.3: Probabilidades de transição $P_{H}(E)$ após t=4 s da explosão e diferentes ângulos de mistura. Em cada quadro estão representadas também as componentes $P_{1,2, s, 3}$. 

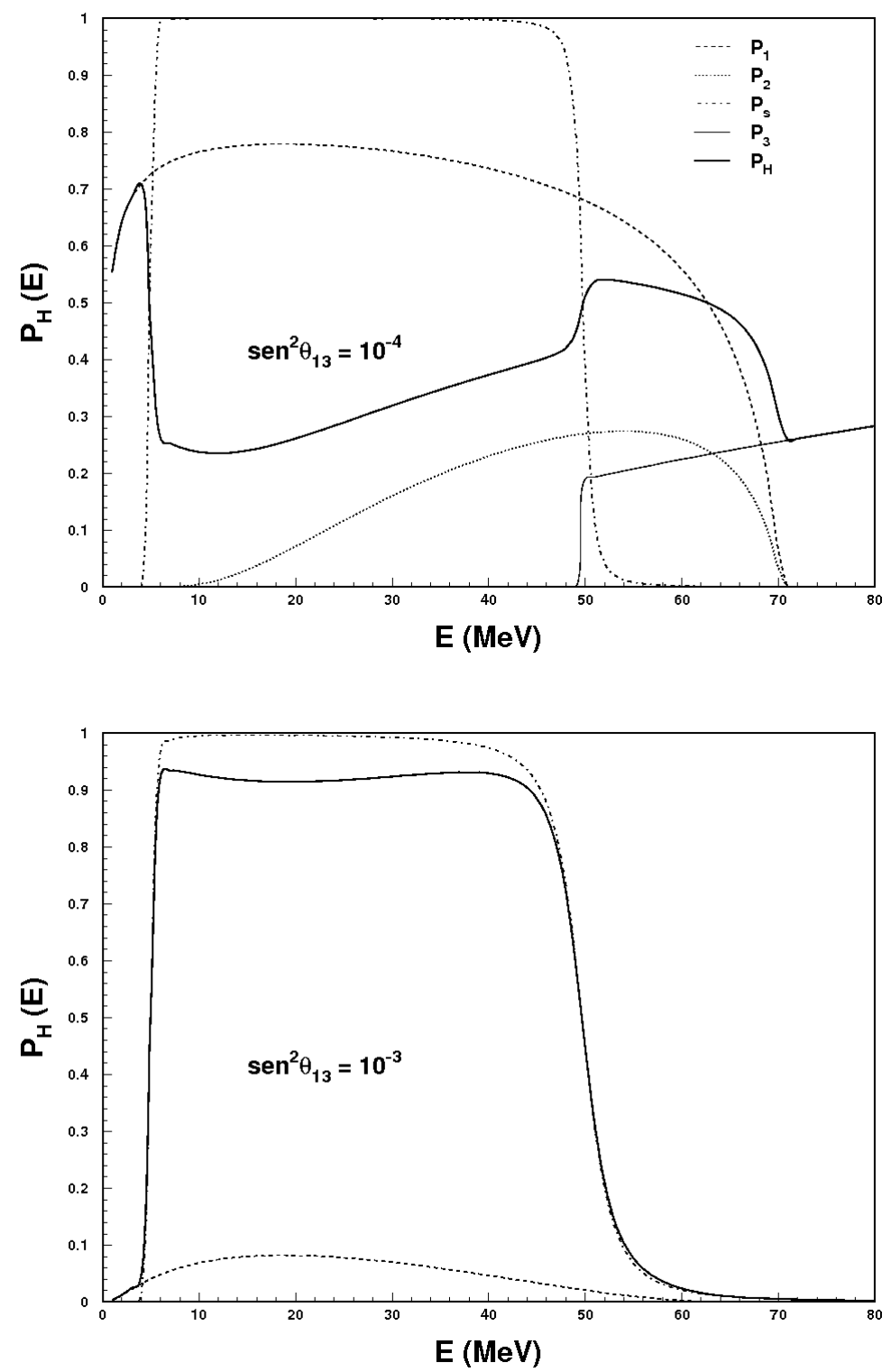

Figura 5.4: Probabilidades de transição $P_{H}(E)$ após t=4 s da explosão e diferentes ângulos de mistura. Em cada quadro estão representadas também as componentes $P_{1,2, s, 3}$. 


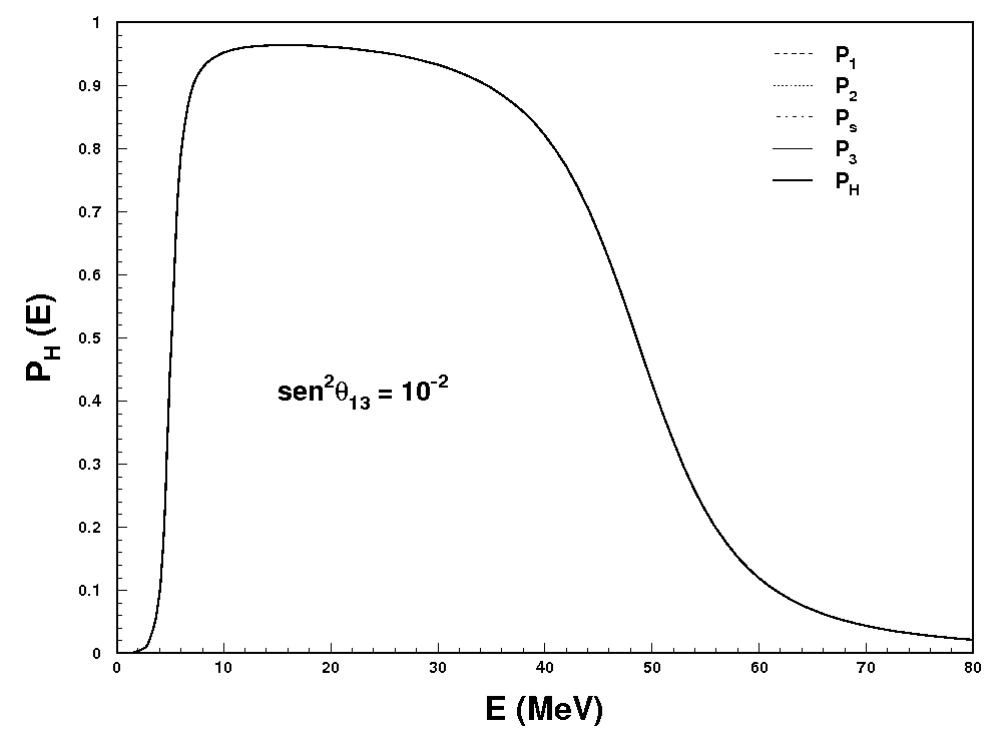

Figura 5.5: Probabilidades de transição $P_{H}(E)$ após t=4 s da explosão e ângulo de mistura indicado. Estão representadas também as componentes $P_{1,2, s, 3}$.

Logo em uma primeira observação é possível verificar que $P_{s}$ (probabilidade de transição na frente de onda) tem um comportamento de "cartola", ou seja, cai rapidamente de $\sim 1$ a 0 para $k_{H}$ fora do intervalo $\left[V^{-}, V^{+}\right]$, ou $\mathrm{V}$ fora do intervalo $[5,50] \mathrm{MeV}$, tendo um comportamento que depende suavemente de $\operatorname{sen}^{2} \theta_{13}$.

Já as probabilidades de Landau-Zener (LZ) $P_{1,2,3}$ são suprimidas exponencialmente com o aumento de $\operatorname{sen}^{2} \theta_{13}$, que é o que acontece quando fazemos uma análise sem onda de choque. Desta forma, para maiores valores de $\operatorname{sen}^{2} \theta_{13}, P_{H}(E)$ é dominado por $P_{s}$, com contribuições quase insignificantes de $P_{1,2,3}$.

Ao contrário, para $\operatorname{sen}^{2} \theta_{13}$ pequenos, como $10^{-6}$, as probabilidades de $P_{1,2,3}$ se tornam próximas a 1. Para diferentes intervalos de energia, que se determinam a partir das energias típicas dos pontos de ressonância, a probabilidade de transição terá diferentes componentes dominantes:

- Para $\mathrm{E} / \mathrm{MeV}<5$, apenas $P_{1}$ é ativo, ou seja, há apenas 1 transição exatamente após a frente de onda.

- Para $5<\mathrm{E} / \mathrm{MeV}<50$, além de $P_{s}, P_{1}$ e $P_{2}$ também são próximos a 1 , tendo assim duas transições na zona de rarefação.

- Para $50<\mathrm{E} / \mathrm{MeV}<67, P_{3}$ também fica ativo e $P_{s}$ cai a zero.

- Para E/MeV $>67$, apenas $P_{3}$ continua significante, mantendo assim apenas as transições antes da passagem da onda de choque.

Para valores de $\operatorname{sen}^{2} \theta_{13}$ intermediários, a análise fica mais complicada pois as probabilidades de transição tomam valores intermediários. No caso de $\operatorname{sen}^{2} \theta_{13}=10^{-5}$, vemos que $P_{1}$ e $P_{3}$ são 
fortemente não adiabáticos $(\sim 1)$ nos extremos de energia, de forma que para baixas e altas energias constituem as únicas contribuições para transição respectivamente, temos $P_{H} \sim 1$. Vemos assim que $P_{H}(E)$ passa pelos valores de $P_{1}, P_{2}$ e $P_{3}$ enquanto a energia passa pelos valores críticos. Já para $\operatorname{sen}^{2} \theta_{13}=10^{-4}$ temos uma análise parecida, ressaltando que neste caso $P_{2}$ pequeno implica que o ponto de rarefação após a frente de onda não é muito relevante no cálculo geral.

Em suma, para um potencial fixo, variações em $\theta_{13}$ modificam significantemente as contribuições de $P_{1}, P_{2}$ e $P_{3}$. Apenas $P_{s}$ se mantem estável e não adiabático. Com uma futura determinação de $\theta_{13}$ será possível estimar a importância relativa de cada contribuição as transições na supernova.

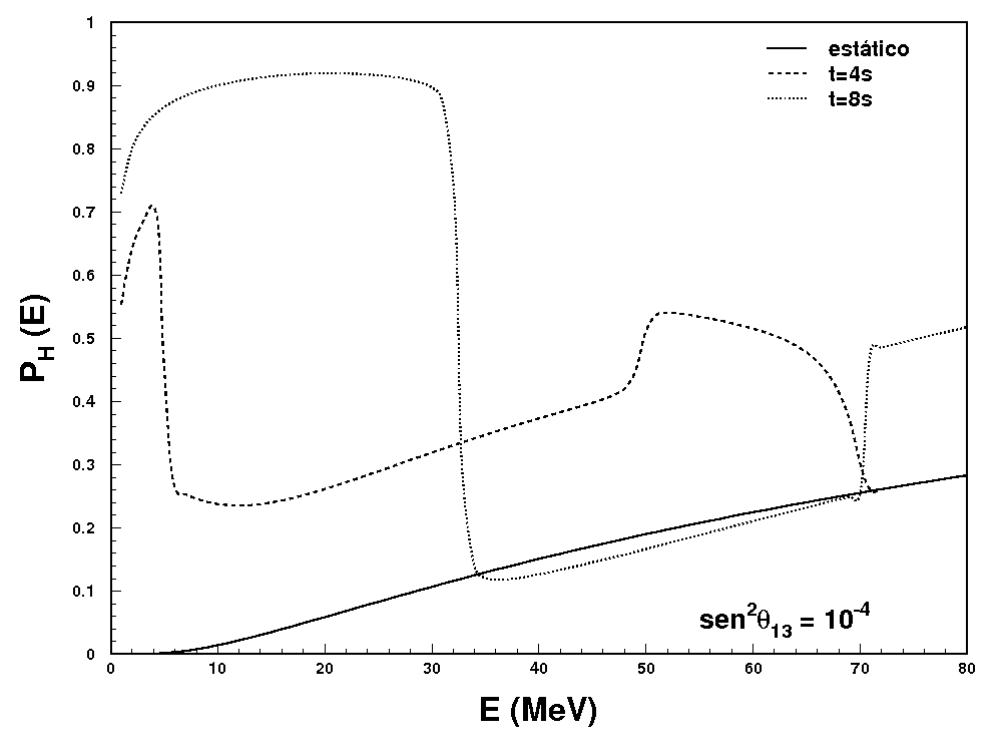

Figura 5.6: $P_{H}(E)$ após $t=0,4$ e $8 \mathrm{~s}$ para $\operatorname{sen}^{2} \theta_{13}=10^{-4}$.

Também podemos olhar para a função $P_{H}(E)$ em tempos diferentes. Na Fig. (5.6) vemos um exemplo para $t=0,4$ e $8 \mathrm{~s} \mathrm{e} \operatorname{sen}^{2} \theta_{13}=10^{-4}$.

Para tempos muito pequenos, a forma de $P_{H}(E)$ é simples, sendo governada pela transição de LZ para um potencial estático. Para tempos maiores, as energias críticas $k_{H}=V^{+}, V^{-}$e $V\left(x_{s}\right)$ avançam e parcialmente saem fora do intervalo de energias relevante para neutrinos de supernovas. Desta forma, a não adiabaticidade referente a transição em $x_{1}$ toma conta de aproximadamente a primeira metade do intervalo de energia, caindo rapidamente quando a transição $P_{2}$ se torna mais relevante. Vemos assim que valores de $P_{H}$ possuem uma estrutura temporal interessante, como será visto com mais detalhes adiante.

\subsubsection{Dependência temporal da probabilidade de transição}

Neste ponto, a energia do neutrino será fixada em $\mathrm{E}=30 \mathrm{MeV}$ e acompanharemos o comportamento de $P_{H}$ em tempos diferentes para os mesmos valores de $\theta_{13}$ utilizados anteriormente (Fig. 
(5.7)). Analogamente ao comportamento com a energia, a estrutura de $P_{H}$ é caracterizada por diferentes regimes ao longo do tempo. Os tempos críticos, que separam cada um destes regimes são facilmente identificados, sendo que seus valores serão diferentes para $E \neq 30 \mathrm{MeV}$.

Novamente as probabilidades $P_{1,2, s, 3}$ desempenharão contribuições diferentes no valor final de $P_{H}(t)$. Para $t<3,2 \mathrm{~s}$ o perfil de densidades é essencialmente estático, $P_{H} \simeq P_{3} \propto$ const $^{\mathrm{sen}^{2} \theta_{13}}$, assim como para $t>7,7 \mathrm{~s}, P_{H} \simeq P_{1} \propto$ const $^{\operatorname{sen}^{2} \theta_{13}}$. Em tempos intermediários as componentes se misturam, tornando a análise menos óbvia. Para $t \simeq 3,2-3,3 \mathrm{~s}$, as probabilidades de transição $P_{2} \mathrm{e}$ $P_{s}$ saem do zero sucessivamente, levando a uma estrutura local complexa. No intervalo $3,3<t / s<$ 7,7, $P_{s}$ é sempre não adiabático, $P_{3}$ pode ser desconsiderado e $P_{1}$ e $P_{2}$ possuem maior relevância para $\operatorname{sen}^{2} \theta_{13}$ pequeno. Os casos mais complicados ocorrem para tempos intermediários e ângulos de mistura $\theta_{13}$ intermediários, dependendo sensivelmente dos valores de $P_{1}$ e $P_{2}$ na zona de rarefação, uma vez que $P_{s} \sim 1$. Os resultados confirmam que variações em $\theta_{13}$ podem causar diferenças grandes na probabilidade de transição como função da energia e do tempo. 

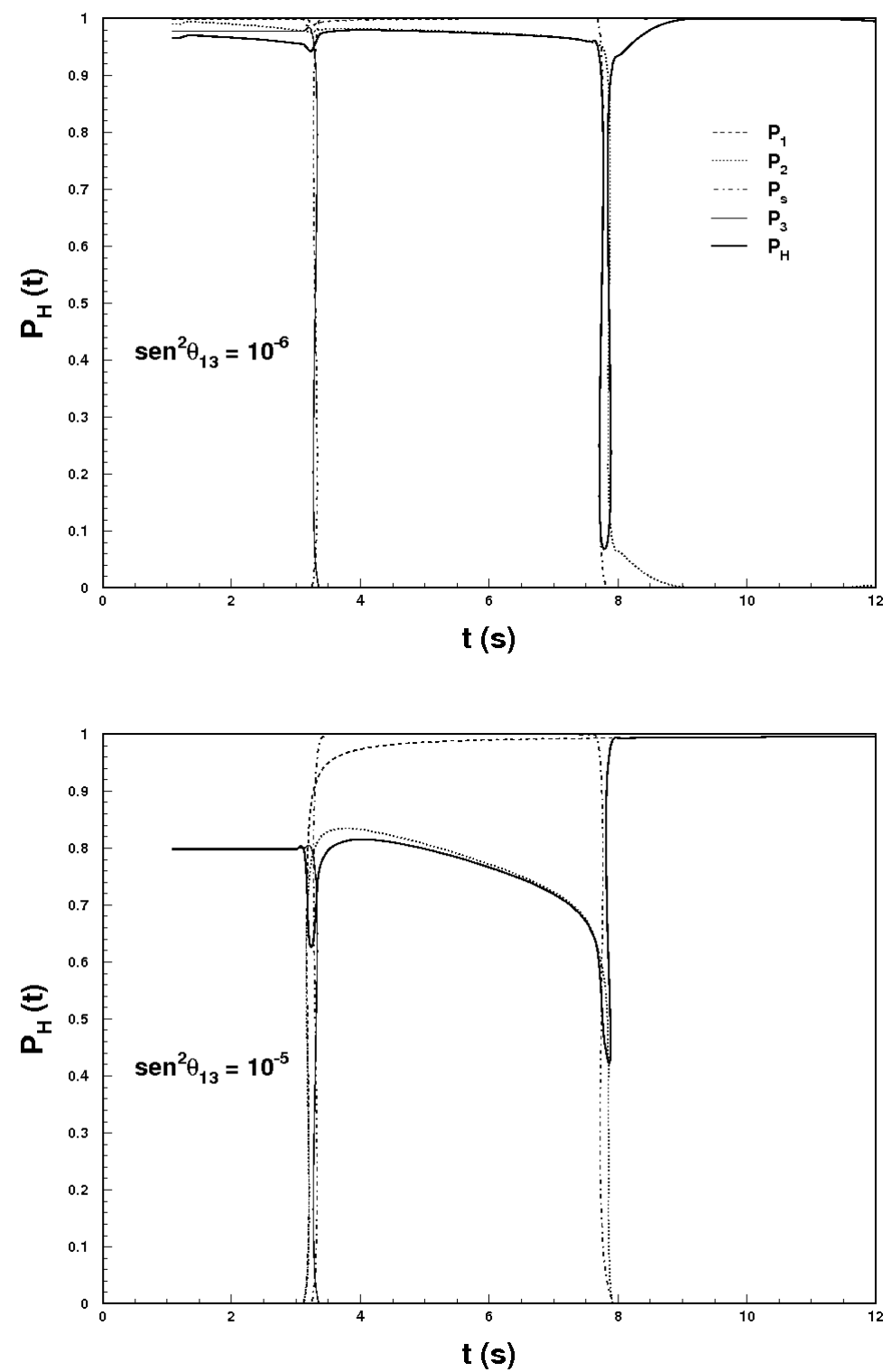

Figura 5.7: Probabilidades de transição $P_{H}(t)$ para $\mathrm{E}=30 \mathrm{MeV}$ e diferentes ângulos de mistura. Em cada quadro estão representadas também as componentes $P_{1,2, s, 3}$. 

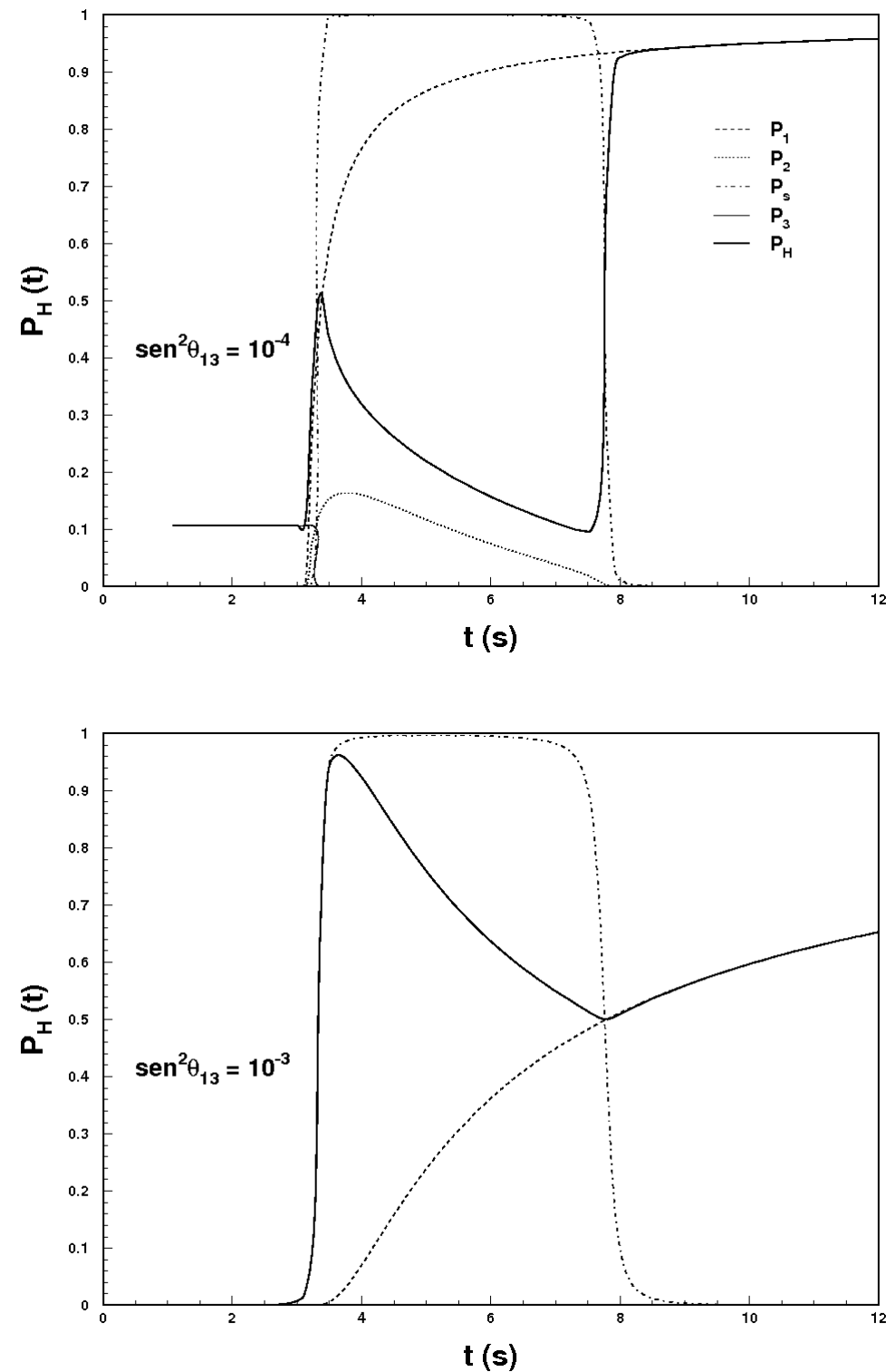

Figura 5.8: Probabilidades de transição $P_{H}(t)$ para $\mathrm{E}=30 \mathrm{MeV}$ e diferentes ângulos de mistura. Em cada quadro estão representadas também as componentes $P_{1,2, s, 3}$. 


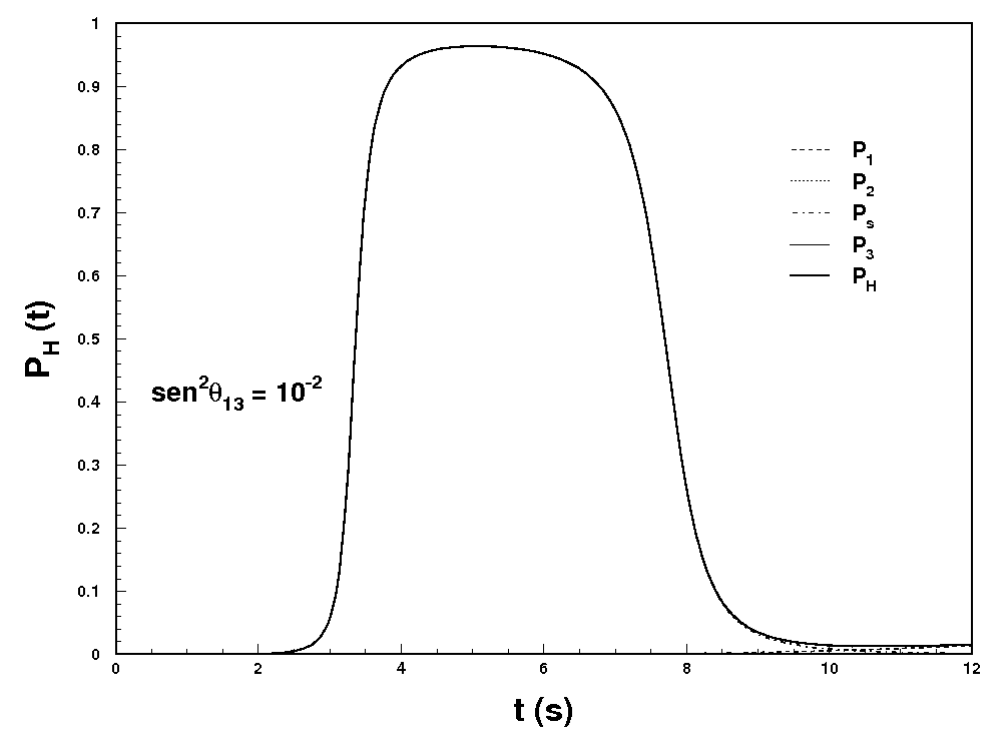

Figura 5.9: Probabilidades de transição $P_{H}(t)$ para $\mathrm{E}=30 \mathrm{MeV}$ e ângulos de mistura indicado. Estão representadas também as componentes $P_{1,2, s, 3}$.

\subsection{Espectro observado}

Assim como no capítulo anterior, no qual foi feita a simulação do espectro e do número de eventos para o caso de uma supernova sem onda de choque, será feita aqui a mesma simulação para hierarquia normal e inversa considerando a probabilidade de transição de neutrinos de supernova com o potencial não estático já descrito. Os intervalos de parâmetros, fluxo e luminosidade, seção de choque e dados do detector serão mantidos para fim de comparação de resultados.

Na Fig. (5.10) vemos o espectro de neutrinos para as transições calculadas em hierarquia normal e inversa, já com o potencial não estático. Juntamente estão os casos extremos sem oscilação, $P_{\bar{e}}=1$, e oscilação total, $P_{\bar{e}}=0$. Nas transições parciais o espectro é basicamente uma combinação linear dos dois casos extremos. Para hierarquia normal, os coeficientes da combinação não dependem da energia, Eq. (4.5), ao contrário do que acontece na hierarquia inversa, Eq. (4.7), em que há uma dependência temporal e energética através de $P_{H}$. Nesta figura, como o tempo foi fixado na integração em um intervalo $[t=6 \pm 0,25 \mathrm{~s}$ ], é a dependência energética que domina o espectro.

No espectro de hierarquia inversa aparecem algumas deformações que se acentuam para ângulos de mistura intermediários, de perfil adiabático. Contudo quando trabalhamos em regiões fortemente não adiabáticas, $\operatorname{sen}^{2} \theta_{13}=10^{-6}$, estas deformações desaparecem, pois sendo $P_{H} \simeq 1$, não se diferenciam do potencial estático. 

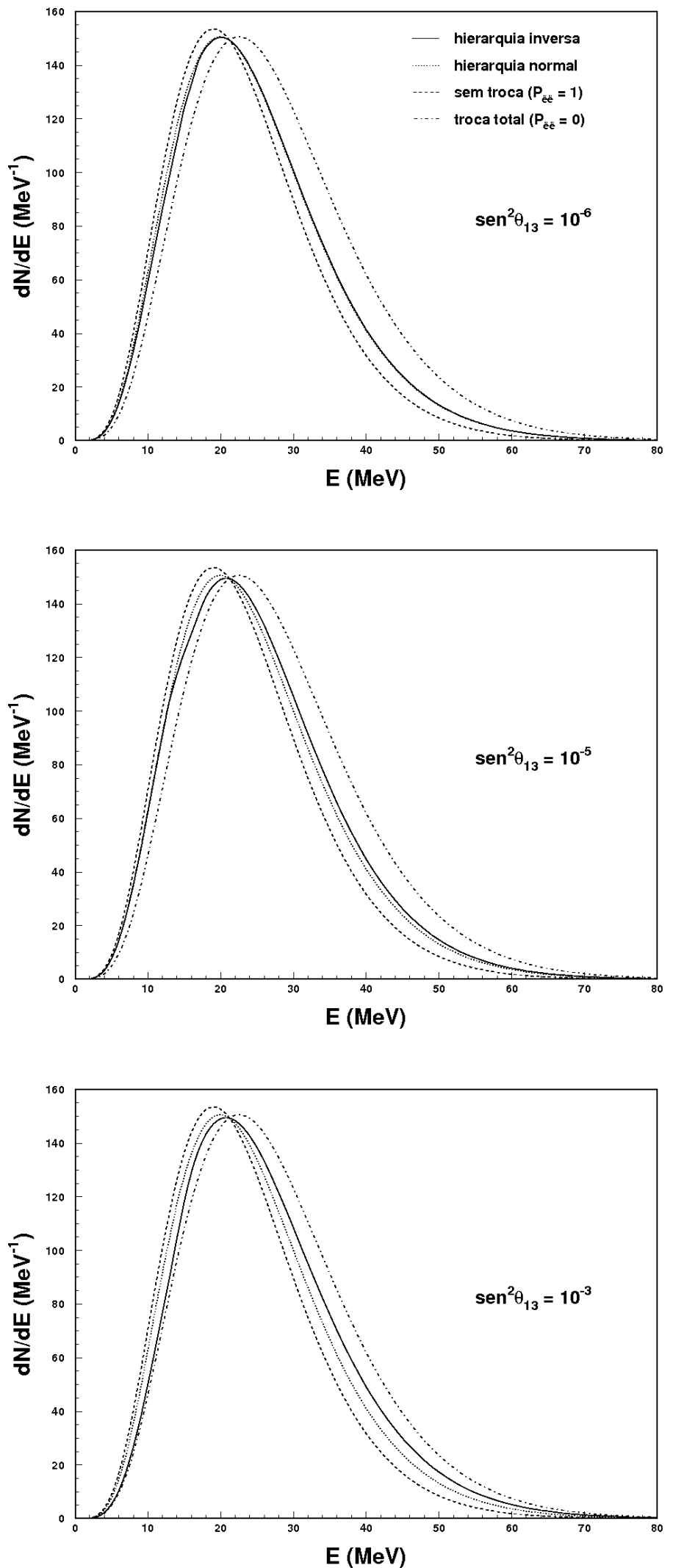

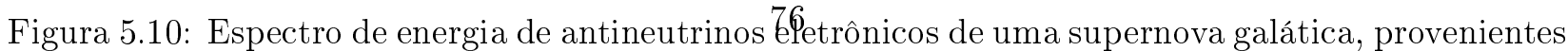
de decaimento beta inverso, no intervalo de tempo $t=6 \pm 0,25 \mathrm{~s}$ e $\operatorname{sen}^{2} \theta_{13}=10^{-6}, 10^{-5}$ e $10^{-3}$ respectivamente. 

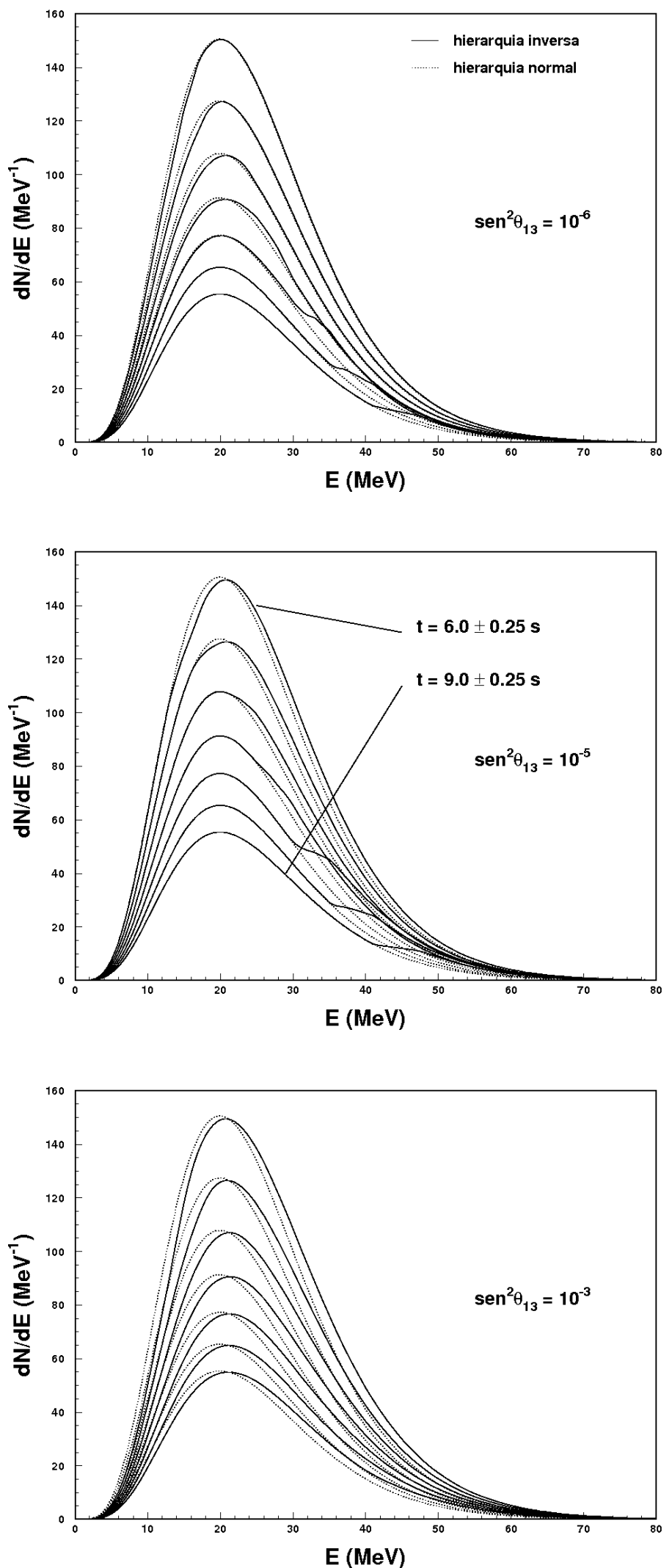

Figura 5.11: Espectro de energia de antineutriñós eletrônicos de uma supernova galática a cada $0,5 \mathrm{~s}$ de 6 a $9 \mathrm{~s}$ após a explosão. Os dados são provenientes de decaimento beta inverso para diferentes valores de ângulo de mistura. Os pontos correspondem a hierarquia normal e a linha cheia a hierarquia inversa. 
Esta deformação também é esperada para outros intervalos de tempo. Na Fig. (5.11) acompanhamos sucessivos espectros a cada $0,5 \mathrm{~s}$ de 6 a $9 \mathrm{~s}$ após a explosão. O espectro como um todo cai ao longo do tempo devido a queda da luminosidade. Consideramos $\operatorname{sen}^{2} \theta_{13}=10^{-6}$ (ângulo pequeno) $10^{-5}$ e $10^{-3}$ (ângulo grande) respectivamente. Podemos ver a dependência temporal que a passagem da onda de choque causa no espectro final para a hierarquia inversa. Ao longo do tempo, a estrutura formada segue para maiores energias, podendo ser feito um paralelo com a Fig. (5.6), em que a estrutura de energias críticas de $P_{H}$ se desloca para maiores energias.

Esta assinatura da onda de choque ao longo do tempo leva a crer que, em uma futura detecção, poderá não ser necessária uma estatística muito grande para a observação desta estrutura temporal.

Uma vez entendido o comportamento das probabilidades de transição e do espectro gerado, queremos obter a quantidade esperada de neutrinos detectados no caso de uma futura explosão de supernova, Eq. (4.10). Novamente assumiremos uma eficiência de $100 \%$ acima do limiar de energia de $5 \mathrm{MeV}$, simulando um tempo de observação de 12 segundos. O resultado final, para potencial estático e não-estático, pode ser encontrado na Tabela 5.1:

\begin{tabular}{|c|c|c|c|c|}
\hline Caso & $N$ (V estático) & $N$ (V não-estático) & $|\Delta N|$ & $5 \sigma$ \\
\hline$\theta_{13}$ grande e HI & 178151 & 175894 & 2257 & \pm 2110 \\
\hline$\theta_{13}$ grande e HN & 160969 & 160970 & 1 & \pm 2006 \\
\hline$\theta_{13}$ pequeno e HI & 161053 & 164740 & 3687 & \pm 2006 \\
\hline$\theta_{13}$ pequeno e HN & 160952 & 160953 & 1 & \pm 2006 \\
\hline
\end{tabular}

Tabela 5.1: Número esperado de eventos de supernova em um detector Cherenkov de 540 kton para reação de decaimento beta inverso referente a um ponto no espaço de parâmetros definido na Tabela (4.1). Vemos aqui a comparação de resultados para o caso sem onda de choque (V estático), com onda de choque ( $\mathrm{V}$ dinâmico), a variação absoluta da quantidade de eventos esperada por cada modelo e o número de eventos correspondendo a uma diferença de $5 \sigma$ a partir do potencial estático.

Diferentemente do que acontece para o caso de potencial estático, aqui não é esperado um mesmo número de eventos para hierarquia normal e inversa para ângulo de mistura pequeno. Isso porque sendo $P_{H} \neq 1$ as probabilidades de sobrevivência não serão iguais para os dois casos. Ponto importante no resultado obtido para o número total de eventos (antineutrinos) corresponde a grande diferença encontrada para hierarquia inversa em modelos de supernova com ou sem onda de choque. Esta diferença é maior que $5 \sigma(\sigma=\sqrt{N})$ e pode sinalizar uma possível forma de determinação da hierarquia. 

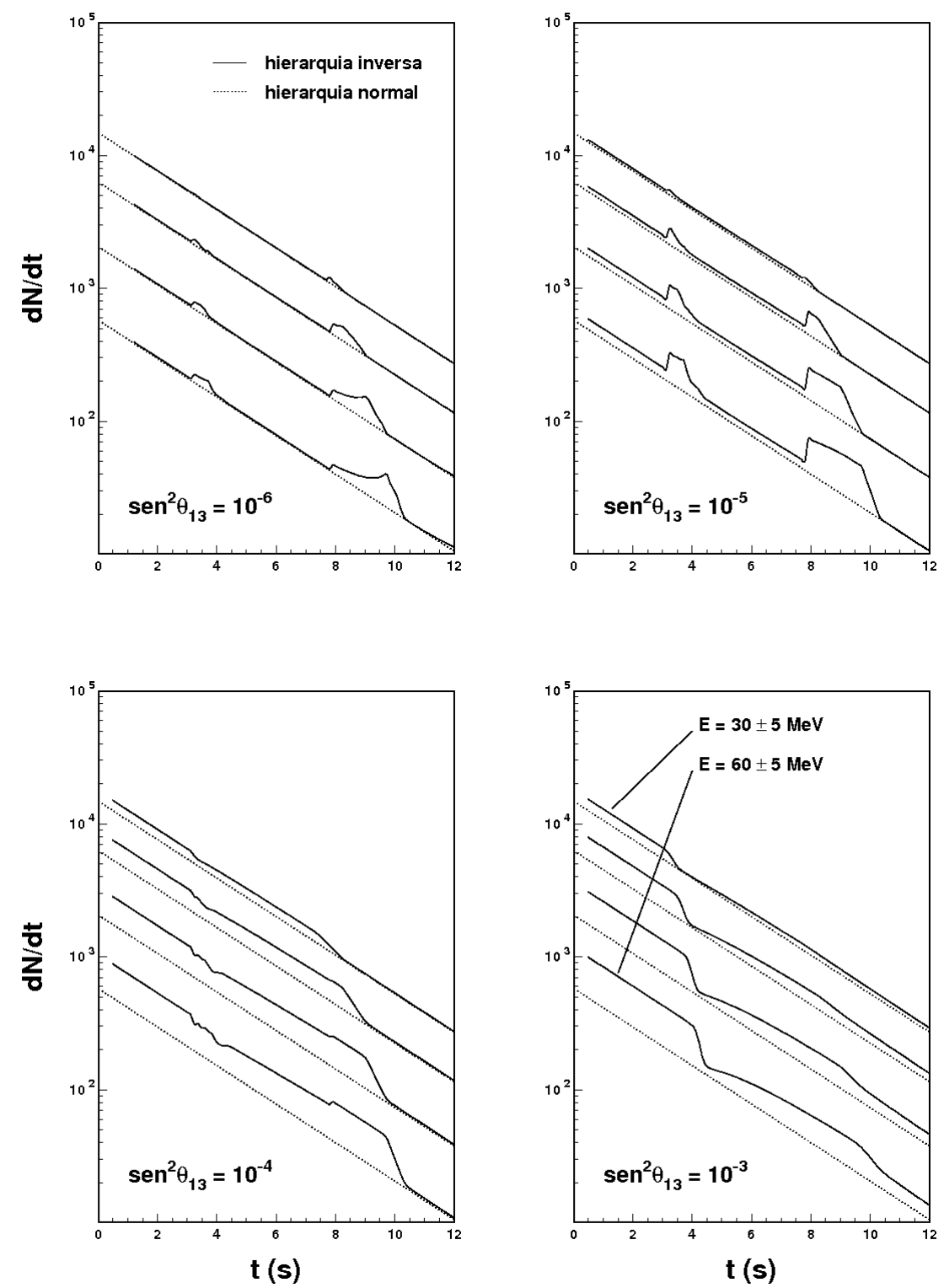

Figura 5.12: Dependência temporal do número de eventos de supernova para diferentes intervalos de energia (de cima para baixo: $30 \pm 5,40 \pm 5,50 \pm 5$ e $60 \pm 5 \mathrm{MeV}$ ). Os pontos correspondem a hierarquia normal e as linhas a hierarquia inversa. Os quadros correspondem respectivamente aos ângulos de mistura: $\operatorname{sen}^{2} \theta_{13}=10^{-6}, 10^{-5}, 10^{-4}$ e $10^{-3}$.

Enquanto a onda de choque atravessa a ressonância, a adiabaticidade é severamente afetada, fazendo com que oscilações sejam suprimidas temporariamente [152]. Após a passagem da onda, as oscilações são retomadas. Desta forma, é esperada uma queda no número de eventos e energia média do neutrino. Este efeito é visível nos canais de neutrino (antineutrino) para hierarquia normal (inversa). No nosso caso, que estamos trabalhando com antineutrinos eletrônicos, observamos uma queda no número de eventos a ser detectado para hierarquia inversa e grande ângulo de mistura. 
Este efeito aparece apenas para oscilações que ocorrem adiabaticamente, $\operatorname{sen}^{2} \theta_{13}>10^{-5}$, Fig. (2.6), no potencial estático. Para o caso de oscilação não-adiabática observamos um aumento no número de eventos, talvez pelo fato de que $P_{H}$ não seja exatamente não-adiabático, mas possui leves flutuações ao longo do intervalo de energia do espectro.

Na Fig. (5.12) acompanhamos a quantidade de antineutrinos emitidos, $d N / d t$ ao longo do tempo de observação, sempre no caso de potencial não-estático. Fica claro como ocorre a variação da emissão de neutrinos para cada uma das hierarquias e como elas tendem a se igualar para o caso de ângulos de mistura muito pequenos. Todos estes sinais da diferença entre as emissões de supernova para hierarquia normal e inversa podem ser a grande chave de uma futura determinação da hierarquia e de um menor limite para o ângulo de mistura $\theta_{13}$.

Nesta parte do trabalho, em que estamos trabalhando com potencial dinâmico, não reproduzimos o cálculo do $\chi^{2}$ para encontrar novos limites de parâmetros, uma vez que o cálculo é essencialmente idêntico ao anterior. Além disto, como vemos na Tabela (5.1), a diferença esperada no fluxo detectado é pequena, principalmente se lembrarmos que não foi considerada na análise a eficiência do detector. Desta forma, esperamos um resultado equivalente que não traria ganho nos limites finais.

É importante salientar que, apesar de pouca diferença de fluxo, a relevância desta parte final do trabalho refere-se a dependência temporal e a forma como esta pode fornecer sinais importantes, ou mesmo definir, a hierarquia de massa dos neutrinos. Em ângulos de mistura intermediários, $\operatorname{sen}^{2} \theta_{13} \approx 10^{-4}-10^{-5}$, a diferença do fluxo esperado para detecção em cada hierarquia é mais acentuada. Nos demais casos pode ser mais complicado, dependendo da sensibilidade do detector, observar possíveis distorções no espectro devido a hierarquia.

Enfim, o trabalho mostra que a detecção de neutrinos provenientes de uma explosão de SN futura pode ser bastante importante na determinação de parâmetros de neutrinos, oscilação e de supernovas. 


\section{Conclusões}

O objetivo inicial do trabalho foi estimar o número de neutrinos que poderiam ser detectados na Terra após a explosão de uma supernova galática e avaliar como este número varia com diferentes parâmetros.

Embora o mecanismo de explosão de supernovas seja pouco conhecido, há diversos modelos e simulações na literatura $[140,149,152,153]$ que permitem avaliar o fluxo de neutrinos dos diversos sabores produzidos no processo de explosão. As diversas simulações produzem luminosidades em neutrinos e valores médios para as energias dos neutrinos que por sua vez deverão ser reconstruídos pelas medidas do fluxo de neutrinos realizadas por detectores terrestres. Isso, em princípio, permitirá determinar quais desses modelos/simulações são mais realistas. Estudaremos a dependência do fluxo de neutrinos em função dos seguintes parâmetros: $\left\langle E_{e}\right\rangle,\left\langle E_{\bar{e}}\right\rangle,\left\langle E_{x}\right\rangle, E_{b} / D^{2}, \operatorname{sen}^{2} \theta_{13},\left|\Delta m_{31}^{2}\right|$ e hierarquia.

Verificamos primeiramente o comportamento do espectro de neutrinos a partir de uma supernova de potencial estático e luminosidade constante, trabalhando a detecção via decaimento beta inverso em um detector Cherenkov. Fizemos o cálculo da seção de choque e das probabilidades de sobrevivência para os dois casos considerados: totalmente adiabático e não-adiabático. A partir disto calculamos o espectro de antineutrinos eletrônicos e o número total esperado para uma detecção na Terra. Estudamos como esses números variam com os parâmetros necessários para descrever a dinâmica da supernova e os parâmetros relevantes da oscilação de neutrinos.

Posteriormente este trabalho foi modificado, e passamos a utilizar uma luminosidade com dependência temporal. Esta modificação foi feita com o objetivo de adequar os resultados obtidos para uma comparação direta com o estudo de uma supernova de potencial dinâmico. Mostramos como é possível encontrar limites de parâmetros de supernova a partir de uma medição do fluxo de antineutrinos na Terra e como a hierarquia de massa dos neutrinos interfere nos resultados. Concluimos que, apesar de no caso de hierarquia inversa e ângulo de mistura grande, $\operatorname{sen}^{2} \theta_{13}=10^{-3}$, não haver sensibilidade com relação a energia média dos antineutrinos eletrônicos, para a energia média de neutrinos não eletrônicos há uma maior sensibilidade.

Uma vez finalizado o trabalho com potencial estático partimos para o cálculo do mesmo número de eventos de antineutrinos, considerando que a explosão gere uma onda de choque, de forma que o potencial tenha uma dependência temporal modificando todo o processo de oscilação. As probabilidades de transição que antes se resumiam a casos totalmente adiabático e não-adiabático agora terão um comportamento bastante diverso. O cálculo numérico agora é bem mais envolvente pois é necessário levar em consideração além da dependência energética uma evolução temporal não trivial. Principalmente vimos que, com a evolução da onda de choque, diferentes pontos de ressonância apa- 
recem ao longo da distância radial do centro da supernova. Estes pontos de maior oscilação causarão variações no fluxo temporal para diferentes hierarquias e ângulos de mistura, de forma que, em uma futura detecção pode ser possível identificar a existência ou não destas distorções e consequentemente um melhor ajuste dos parâmetros de oscilação.

Finalmente, com estes resultados em mãos pudemos comparar os resultados esperados para o espectro de uma supernova quando consideramos potencial estático ou dinâmico. Quando observamos o número de eventos esperado em cada caso, notamos que, apesar de entre os resultados haver uma diferença de mais de $5 \sigma$, este número é pequeno se pensarmos em uma detecção realista. Consideramos aqui apenas um canal de deteç̧ão e 100 \% de eficiência do detector, o que não reproduz condições reais de detecção e infelizmente parece difícil nessas condições concluir se o detector estudado poderá realmente ter sensibilidade a diferenças relativas à dinâmica do potencial. Muitas das simplificações aqui feitas podem ser removidas, o que permitirá uma estimativa mais realista sobre a possibilidade real de utilizar neutrinos de supernova para estudar a dinâmica da explosão. No entanto, a não ser que outros canais sejam observados, não esperamos que um estudo mais realista melhore os resultados aqui obtidos. 


\section{Referências Bibliográficas}

[1] Paticle Data Group - http://pdg.lbl.gov/.

[2] H. Bethe, R. Peierls, The "neutrino", Nature 133 (1934) 532.

[3] C. L. Cowan Jr., F. B. Harrison, H. B. Kruse, A. D. McGuire, Science 124 (1956) 103.

F. Reines, C. L. Cowan Jr., Nature 178 (1956) 446.

[4] B. Pontecorvo, Sov. Phys. JETP 26 (1968) 984.

[5] V. N. Gribov, B. Pontecorvo, Phys. Lett. B28 (1969) 493.

[6] G. Danby, J. M. Gaillard, K. Goulianos, L. M. Lederman, N. Mistry, M. Schwartz, J. Steinberger, Phys. Rev. Lett. 9 (1962) 36.

[7] K. Kodama et. al, Phys. Lett. B504 (2001) 218.

[8] A. S. Dighe, A. Y. Smirnov, Phys. Rev. D62 (2000) 033007.

[9] C. Quigg, Gauge theories of strong, weak and eletromagnetic interactions, Benjamin/Cummings Pub. Co. (1983).

[10] E. Majorana, Nuovo Cim. 14 (1937) 171.

[11] M. Geell-Mann, P. Rammond, R. Slansky, Supergravity, Eds. D. Freedman et al (1980).

[12] G. Hinshaw et al. [WMAP Collaboration], arXiv:0803.0732.

[13] S, R. Elliot, P. Vogel, Annu. Rev. Nucl. Part. Sci. 52 (2002), hep-ph/0202264.

[14] H. V. Klapdor-Kleingrothaus et al., Eur. Phys. J. A12 (2001) 147.

[15] C. Arnaboldi et al., Phys. Rev. Lett. 95 (2005) 142501.

[16] R. Arnold et al. [NEMO Collaboration], Nucl. Phys. A781 (2007) 209.

[17] A. Barabash [NEMO Collaboration], arXiv: 0807.2336.

[18] J. N. Bahcall, A. M. Serenelli, S. Basu, Astrophys. J. 621 (2005) L85.

[19] R. J. Davis, D. S. Harmer, K. C. Hoffman, Phys. Rev. Lett 20 (1968) 1205.

[20] B. T. Cleveland et al., Astrophys. J. 496 (1998) 505. 
[21] J. N. Abdurashitov et al. [SAGE Collaboration], J. Exp. Theor. Phys. 95 (2002) 181.

[22] W. Hampel et al. [GNO Collaboration], Phys. Lett. B447 (1999) 127.

[23] M. Altmann et al. [GNO Collaboration], Phys. Lett. B616 (2005) 174.

[24] Y. Fukuda et al. [Kamiokande Collaboration], Phys. Rev. Lett. 77 (1996) 1683.

[25] M. B. Smy et al. [Super-Kamiokande Collaboration], Phys. Rev. D69 (2004) 011104.

[26] Q. R. Ahmad et al. [SNO Collaboration], Phys. Rev. Lett. 87 (2001) 071301.

[27] Q. R. Ahmad et al. [SNO Collaboration], Phys. Rev. Lett. 89 (2002) 011301.

[28] Q. R. Ahmad et al. [SNO Collaboration], Phys. Rev. Lett. 89 (2002) 011302.

[29] S. N. Ahmed et al. [SNO Collaboration], Phys. Rev. Lett. 92 (2004) 181301.

[30] B. Aharmim et al. [SNO Collaboration], Phys. Rev. C72 (2005) 055502.

[31] L. Oberauer, Nucl. Phys. Proc. Suppl. 77 (1999) 48.

[32] Borexino Collaboration, arXiv: 0708.2251.

[33] F. Reines et al., Phys. Rev. Lett. 15 (1965) 429.

[34] C. V. Achar et al., Phys. Lett. 18 (1965) 196.

[35] R. Becker-Szendy et al., Phys. Rev. D46 (1992) 3720.

[36] Y. Fukuda et al., [Super-Kamiokande Collaboration], Phys. Rev. Lett. 81 (1998) 1562.

[37] M. C. Sanchez et al., [Soudan 2 Collaboration], Phys. Rev. D68 (2003) 113004.

[38] M. Ambrosio et al., [MACRO Collaboration], Phys. Lett. B517 (2001) 59.

[39] Y. Ashie et al., [Super-Kamiokande Collaboration], Phys. Rev. D71 (2005) 112005.

[40] J. Hosaka et al., [Super-Kamiokande Collaboration], Phys. Rev. D74 (2006) 032002.

[41] P. J. Litchfield, The XXII International Conference on Neutrino Physics, Santa Fe, Novo Mexico, 13-19 de Junho de 2006.

[42] G. Zacek et al., [CALTECH-SIN-TUM Colaboration], Phys. Rev. D34 (1986) 2621.

[43] G. S. Vidyakin et al., JETP Lett. 59 (1994) 390.

[44] Y. Declais et al., Nucl. Phys. B434 (1995) 503.

[45] M. Apollonio et al., [CHOOZ Collaboration], Phys. Lett. B466 (1999) 415.

[46] A. Piepke [Palo Verde Collaboration], Prog. Part. Nucl. Phys. 48 (2002) 113.

[47] K. Eguchi et al. [KamLAND Collaboration], Phys. Rev. Lett. 90 (2003) 021802. 
[48] E. Aliu et al. [K2K Collaboration], Phys. Rev. Lett. 94 (2005) 081802.

[49] D. G. Michael et al. [MINOS Collaboration], Phys. Rev. Lett. 97 (2006) 191801.

[50] A. A. Aguilar et al. [LSND Collaboration], Phys. Rev. D64 (2001) 112007.

[51] B. Armbruster et al. [KARMEN Collaboration], Phys. Rev. D65 (2002) 112001.

[52] A. A. Aguilar-Arevalo et al. [MiniBooNe Collaboration], Phys. Rev. Lett. 98 (2007) 231801.

[53] R. N. Mohapatra, P. B. Pal, Massive Neutrinos in Physics and Astrophysics, World Scientific (2004).

[54] T. K. Kuo, J. Pantaleone, Rev. Mod. Phys. 61 (1989) 937.

[55] E. Kh. Akhmedov, Neutrino Physics (2000), hep-ph/0001264.

[56] C. W. Kim, A. Pevsner, Comtemp. Concepts Phys. 8 (1993) 1.

[57] L. Wolfenstein, Phys. Rev D17 (1978) 2369.

[58] S. P. Mikheev, A. Y. Smirnov, Nu. Cim. C9 (1986) 17.

[59] P. B. Pal, IJMP A7 (1992) 5387.

[60] P. Pizzochero, Phys. Rev. D36 (1987) 2293.

[61] S. T. Petcov, Phys. Lett. B200 (1988); M. M. Guzzo, J. Bellandi, V. M. Aquino, Phys. Rev. D49 (1994) 1404.

[62] M. C. Gonzalez-Garcia, Y. Nir, Rev. Mod. Phys. 75 (2003) 345.

[63] M. C. Gonzalez-Garcia, M. Maltoni, Phenomenology with Massive neutrinos,(2007), arXiv:0704.800v2.

[64] J. N. Bahcall, M. C. Gonzalez-Garcia, C. Pena-Garay, JHEP 0108 (2001) 14.

[65] T. Schwetz, M. Tortola, J. W. F. Valle, New J. Phys. 10 (2008) 113011.

[66] S. Abe et al. [KamLAND Collaboration], arXiv:0801.4589.

[67] B. Jamieson et al. [SNO Collaboration], arXiv:0810.3760.

[68] P. Adamson et al. [MINOS Collaboration], arXiv:0711.0769.

[69] S. A. Colgate, R. H. White, Astrophys. J. 143 (1966) 626.

[70] R. L. B. Minkowski, ApJ. 89 (1939) 156;

PASP 53 (1941) 224.

[71] S. Chandrasekhar, Astrophys. J. 74 (1931) 81.

[72] W. Rose, Advanced stellar astrophysics, Cambridge University Press (1998). 
[73] R. J. Tayler, The stars: Their structure and evolution, Cambridge University Press (1994).

[74] E. M. Burbidge, G. R. Burbidge, W. A. Fowler, F. Hoyle, Rev. Mod. Phys. 29 (1957) 547.

[75] G. A. Gamow, M. Schenberg Phys. Rev. 59 (1941) 539.

[76] G. Gamow, M. Schenberg, Phys. Rev. 58 (1940) 1117.

[77] J. I. Zuluaga, Study of core collapse neutrino signals and constraints on neutrino masses from a future Galactic Supernova, astro-ph/0511771.

[78] R. Buras, M. Rampp, H. T. Janka, K. Kifonidis, Phys. Re,v. Lett. 90 (2003).

[79] D. N. Spergel, T. Piran, A. Loeb, J. Goodman, J. N. Bahcall, Science 237 (1987) 1471.

[80] M. T. Keil, Supernova neutrino spectra and applications to flavor oscillations, astro-ph/0308228.

[81] G. C. Raffelt, Astrophys. J. 561 (2001) 890.

[82] A. S. Dighe, A. Y. Smirnov, (1999), hep-ph/9907423.

[83] A. Burrows, D. Klein, R. Gandhi, Phys. Rev. D45 (1992) 3361.

[84] S. E. Woosley, J. R. Wilson, G. J. Mathews, R. D. Hoffman, B. S. Meyer, Astrophys. J. 433 (1994) 229.

[85] T. Totani, Phys. Rev. Lett. 80 (1998) 2039.

[86] J. F. Beacom, R. N. Boyd, A. Mezzacappa, Phys. Rev. D63 (2001) 073011.

[87] G.G. Raffelt, M. T. Keil, R. Buras, H. T. Janka, M. Rampp, astro-ph/0303226.

[88] R. Buras, M. Rampp, H. T. Janka, K. Kifonidis, astro-ph/0507135.

[89] J. F. Beacom, R. N. Boyd, A. Mezzacappa, Phys. Rev. Lett. 85 (2000) 3568.

[90] K. Hirata et al., Phys. Rev. Lett. 58 (1987) 1490.

[91] R. Bionta et al., Phys. Rev. Lett. 58 (1987) 1494.

[92] M. Aglieta et al., Eur. Phys. Lett. 3 (1987) 1321.

[93] L. Wolfenstein, Phys. Lett. B194 (1987) 197.

[94] D. Notzold, Phys. Lett. B196 (1987) 315.

[95] J. Arafune, M. Fukugita, Phys. Rev. Lett. 59 (1987) 367.

[96] J. Arafune, M. Fukugita, T. Yanagida, M. Yoshimura, Phys. Rev. Lett. 59 (1987) 1864.

[97] H. Minakata, H. Nunokawa, K. Shiraishi, H. Suzuki, Mod. Phys. Lett. A2 (1987) 827.

[98] H. Minakata, H. Nunokawa, Phys. Rev. D38 (1988) 3605.

[99] A. Y. Smirnov, D. N. Spergel, J. N. Bahcall, Phys. Rev. D49 (1994) 1389. 
[100] B. Jegerlehner, F. Neubig, G. Raffelt, Phys. Rev. D54 (1203) 1194.

[101] C, Lunardini, A. Y. Smirnov, Phys. Rev. D63 (2001) 073009.

[102] H. Minakata, H. Nunokawa, Phys. Lett. B504 (2001) 301.

[103] M. Kachelriess, A. Strumia, R. Tomas, J. W. F. Valle, Phys. Rev. D65 (2002) 073016.

[104] M. Kachelriess, R. Tomas, J. W. F. Valle, JHEP 01 (2001) 030.

[105] V. Barger, D. Marfatia, B. P. Wood, Phys. Lett. B532 (2002) 19.

[106] G. L. Fogli, E. Lisi, D. Montanino, A. Palazzo, Phys. Rev. D65 (2002) 073008.

[107] F. Buccella, S. Esposito, C.Gualdi, G. Miele, Z. Phys. C73 (1997) 633.

[108] H. Minakata, H. Nunokawa, Phys. Lett. B504 (2001) 301.

[109] G. Dutta, D. Indumathi, M. V. N. Murthy, G. Rajasekaran, Phys. Rev. D61 (2000) 013009.

[110] S. T. Petcov, Phys. Rev. B200 (1988) 373.

[111] P. I. Krastev, S. T. Petcov, Phys. Lett. B207 (1988) 64; B214, (1988) 661.

[112] A. Friedland, Phys. Rev. D64 (2001) 013008.

[113] E. Lisi, A. Marrone, D. Montanino, A. Palazzo, S. T. Petcov, Phys. Rev. D63 (2001) 093002.

[114] G. E. Brown, H. A. Bethe, G. Baym, Nucl. Phys. A375 (1982) 481.

[115] T. Shigeyama, K. Nomoto, Astrophys. J. 360 (1990) 242.

[116] K. Takahashi, W. Watanabe, K. Sato, T. Totani, Phys. Lett. B510 (2001) 189.

[117] K. Takahashi, W. Watanabe, K. Sato, T. Totani, Phys. Rev. D64 (2001) 093004.

[118] K. Takahashi, K. Sato, hep-ph/0110105.

[119] H. Minakata, H. Nunokawa, Phys. Rev. D38 (1988) 3605.

[120] A. De Gouvea, Phys. Rev. D39 (1989) 1930.

[121] M. Kachelriess, R. Tomas, J. W. F. Valle, J. High Energy Phys. 01 (2001) 30.

[122] M. Kachelriess, R. Tomas, Phys. Rev. D64 (2001) 073002.

[123] M. Kachelriess, A. Strumia, R. Tomas, J. W. F. Valle, hep-ph/0108100.

[124] J. Pantaleone, Phys. Lett. B287 (1992) 128.

[125] G. Sigl, G. Raffelt, Nucl. Phys. B406 (1993) 423.

[126] H. Duan, G. M. Fuller, Y. Z. Qian, Phys. Rev. D74 (2006) 123004.

[127] H. Duan, G. M. Fuller, J. Carlson, Y. Z. Qian, Phys. Rev. D74 (2006) 105014. 
[128] S. Hannestad, G. G. Raffelt, S. Sigl, Y. Y. Y. Wong, Phys. Rev. D74 (2006) 105010.

[129] G. G. Raffelt, G. Sigl, Phys. Rev. D75 (2007) 083002.

[130] H. Duan, G. M. Fuller, J. Carlson, Y. Z. Qian, Phys. Rev. D75 (2007) 125005.

[131] G. G. Raffelt, A. Y. Smirnov, Phys. Rev. D77 (2008) 029903.

[132] G. G. Raffelt, A. Y. Smirnov, Phys. Rev. D76 (2007) 125008.

[133] A. Esteban-Pretel, S. Pastor, R. Tomàs, G. G. Raffelt, G. Sigl, Phys. Rev. D76 (2007) 125018.

[134] H. Duan, G. M. Fuller, Y. Z. Qian, Phys. Rev. D76 (2007) 085013.

[135] H. Duan, G. M. Fuller, J. Carlson, Y. Z. Qian, Phys. Rev. Lett. 99 (2007) 241802.

[136] G. L. Fogli, E. Lisi, A. Marrone, A. Mirizzi, JCAP 0712 (2007) 010.

[137] H. Duan, G. M. Fuller, J. Carlson, Y. Z. Qian, Phys. Rev. Lett. 100 (2008) 021101.

[138] A. Esteban-Pretel, S. Pastor, R. Tomàs, G. G. Raffelt, G. Sigl, Phys. Rev. D77 (2008) 065024.

[139] B. Dasgupta, A. Dighe, A. Mirizzi, G. G. Raffelt, arXiv:0801.1660v2 [hep-ph].

[140] S. Skadhauge, R. Z. Funchal, Determining neutrino and supernova parameters with a galactic supernova (2006), hep-ph/0611194.

[141] R. Buras, H. T. Janka, M. Rampp, K. Kifonidis, astro-ph/0512189; G. G. Rafflelt, M. T. Keil, R. Buras, H. T. Janka, M. Rampp, astro-ph/0303226; M. T. Keil, G. G. Rafflelt, H. T. Janka, Astrophys. J. 590 (2003) 971, astro-ph/0208035.

[142] T. Totani, K. Sato, H. E. Dalhed, J. R. Wilson, Astrophys. J. 496 (1998) 216.

[143] G. L. Fogli, E. Lisi, A. Mirizzi, D. Montanino, Phys. Rev. D68 (2003) 033005, hep-ph/0304056.

[144] P. Vogel, F. Beacom, Phys. Rev. D60 (1999) 053003, hep-ph/9903554.

[145] Y. Itow et al., hep-ph/0106019.

[146] C. K. Jung [UNO Collaboration], hep-ex/0005046.

[147] A. Tonazzo [MEMPHYS Collaboration], Nucl. Phys. Proc. Suppl. 168 (2007) 363.

[148] K. Takahashi, K. Sato, Nucl. Phys. A718 (2003) 455.

[149] R. C. Schirato, G. M. Fuller, "Connection between supernova shocks, flavor transformation, and the neutrino signal" astro-ph/0205390.

[150] K. Takahashi, K. Sato, H. E. Dalhed, J. R. Wilson, astro-ph/0212195.

[151] T. K. Kuo, J. Pantaleone, Rev. Mod. Phys. 61 (1989) 937.

[152] V. Barger, P. Huber, D. Marfatia, Phys. Lett. B617 (2005) 167.

[153] G. L. Fogli, E. Lisi, A. Mirizzi, D. Montanino, JCAP 04 (2005) 002. 